\section{Pacific Northwest}

National Laboratory

Operated by Battelle for the

U.S. Department of Energy

\title{
Impact of HLW Glass Crystallinity on the PCT Response
}

B.J. Riley P. Hrma

J.A. Rosario

April 2001

Prepared for the U.S. Department of Energy

under Contract DE-AC06-76RL01830 


\section{DISCLAIMER}

This report was prepared as an account of work sponsored by an agency of the United States Government. Neither the United States Government nor any agency thereof, nor Battelle Memorial Institute, nor any of their employees, makes any warranty, expressed or implied, or assumes any legal liability or responsibility for the accuracy, completeness, or usefulness of any information, apparatus, product, or process disclosed, or represents that its use would not infringe privately owned rights. Reference herein to any specific commercial product, process, or service by trade name, trademark, manufacturer, or otherwise does not necessarily constitute or imply its endorsement, recommendation, or favoring by the United States Government or any agency thereof, or Battelle Memorial Institute. The views and opinions of authors expressed herein do not necessarily state or reflect those of the United States Government or any agency thereof.

\section{PACIFIC NORTHWEST NATIONAL LABORATORY operated by \\ BATTELLE MEMORIAL INSTITUTE for the UNITED STATES DEPARTMENT OF ENERGY under Contract DE-AC06-76RLO 1830}


PNNL-13491

\title{
Impact of HLW Glass Crystallinity on the PCT Response
}

\author{
B. J. Riley \\ J. A. Rosario \\ P. Hrma
}

April 2001

Prepared for the U.S. Department of Energy

under Contract - DE-AC06-76RL01830

Pacific Northwest National Laboratory

Richland, Washington 99352 


\section{Summary}

Optimizing glass formulation for vitrifying waste is based on computing a glass composition that would meet processability and acceptability constraints imposed on glass properties and composition while maximizing system efficiency. The process currently employed allows for estimation of all limiting glass properties except for the normalized element releases $\left(r_{i}\right.$, where $i=\mathrm{B}, \mathrm{Na}$, or $\mathrm{Li}$ ) by the product consistency test (PCT) from glasses subjected to canister centerline cooling (CCC). This report documents a suggested approach to include this key glass property in numerical glass optimization. It shows that acceptable predictions of $r_{B}$ and $r_{N a}$ from CCC glasses can be obtained provided that sufficiently accurate compositions and concentrations of the crystalline phases in CCC glass are known.

The composition, crystallinity, and $r_{B}$ and $r_{N a}$ of 266 glasses were collected and evaluated to determine the relationship between PCT response and crystallinity (measured using semiquantitative $\mathrm{x}$ ray diffraction [XRD]). Glasses were sorted to several groups according to the major crystalline phase that precipitated upon heat-treatment, either CCC or isothermal. A review of previous work done in this area showed that while crystallization may have little or negative impact on PCT response, precipitation of cristobalite and certain aluminosilicates can increase $r_{B}$ and $r_{N a}$ by several orders of magnitude. This behavior is attributed to changes of the residual glass composition associated with crystallization (or, rarely, to phase separation preceding or following crystallization). Attempts were made in the past to predict $r_{i}$ from heat-treated glasses. The results were encouraging. The calculations were based on the application of first-order models for $r_{B}$ and $r_{N a}$ to residual glass compositions (compositions of the amorphous phase separation after the crystalline phases precipitated).

This report collects and discusses existing data on $r_{B}$ and $r_{N a}$ from glasses that precipitated crystalline phases upon CCC or isothermal heat-treatment (IHT). It summarizes compositions of these glasses (grouped by major mineral phases that precipitated during heat treatment), the mass fractions of individual crystalline phases in each glass, and measured PCT releases $\left(r_{B}\right.$ and $\left.r_{\mathrm{Na}}\right)$ from quenched and heat-treated glasses. Residual glass compositions are calculated based on mass fractions of crystalline phases. Two methods of calculation were used to estimate $r_{B}$ and $r_{N a}$ values (one based on estimated and the other on measured $r_{B}$ and $r_{N a}$ from quenched glasses), which are compared with measured data. For each group of glasses, ten plots were generated that compare releases from heat-treated and quenched glasses and calculated and measured $r_{B}$ and $r_{N a}$ values. The results are discussed, and special attention is paid to outliers. The authors suggest that the outliers are caused in most cases by model inaccuracy in composition regions well outside the original database (of quenched glasses) to which the models were fitted.

Methods for application of this approach for estimating $r_{B}$ and $r_{N a}$ of heat-treated glasses are suggested. To be successful, we would suggest that the models for $r_{B}$ and $r_{N a}$ be extended to include residual glass compositions and tools be developed for estimating the concentration and composition of key crystalline phases formed from HLW glasses. 


\section{Glossary}

ASTM American Society for Testing and Materials

CCC canister centerline cooling

CVS composite variability study

DOE U.S. Department of Energy

DWPF Defense Waste Processing Facility

EA environmental assessment

EDS energy dispersive spectroscopy

HLW high-level waste

LAW low-activity waste

IHT isothermal heat treatment

NP nepheline

PCT product consistency test

PNNL Pacific Northwest National Laboratory

SEM scanning electron microscopy

SRTC Savannah River Technology Center

XRD X-ray diffraction 


\section{List of Symbols}

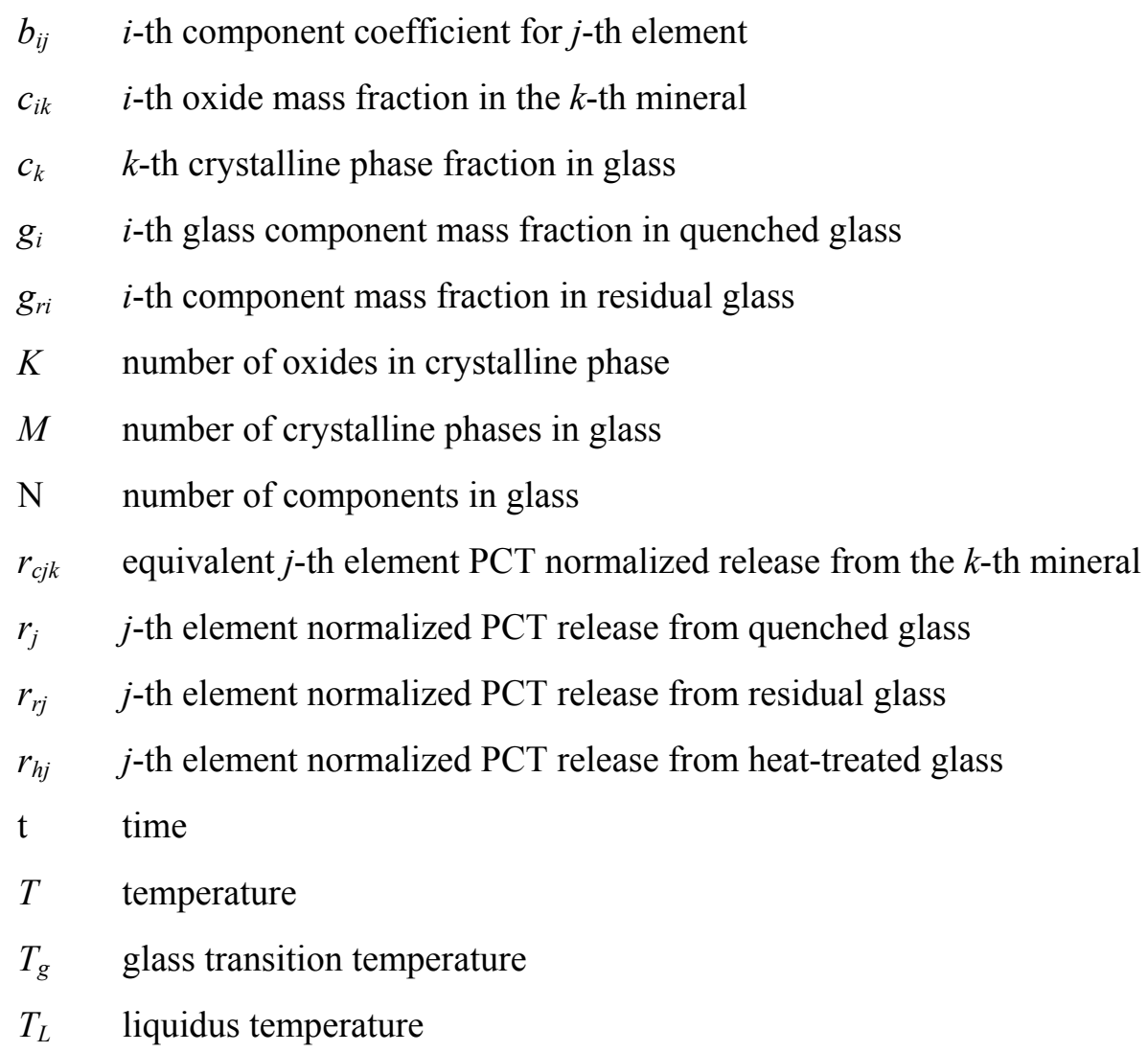

\section{Abbreviations used in tables and figures}

C calculated values of elemental PCT releases

$\mathrm{H}$ heat treated (CCC or IHT) glass

M measured values of elemental PCT releases

Q quenched glass

QC calculated values of elemental PCT releases from quenched glass

QM measured values of elemental PCT releases from quenched glass

RC calculated values of elemental PCT releases from residual glass in heat-treated samples 


\section{Acknowledgments}

The authors thank John Vienna for his insightful advice, Dong Kim and David Peeler for careful review, Wayne Cosby for excellent editing, and Bill Hotzscheiter for kind guidance. Brian Riley, and Jose Rosario are grateful to Associated Western Universities for fellowship appointments at Pacific Northwest National Laboratory, which is operated for the U.S. Department of Energy (DOE) by Battelle under Contract DE-AC06-76RL01830. The DOE funded this work through the Office of Environmental Management's Tanks Focus Area program. 


\section{Contents}

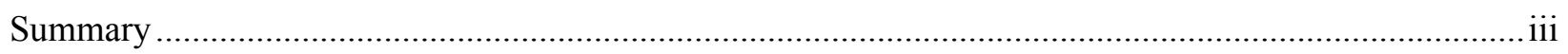

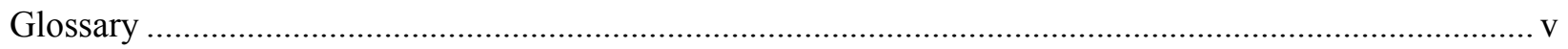

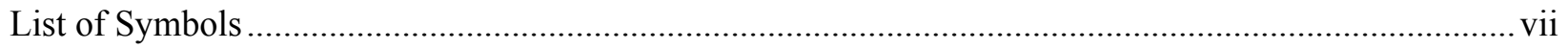

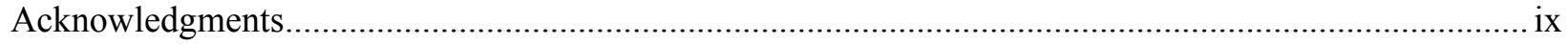

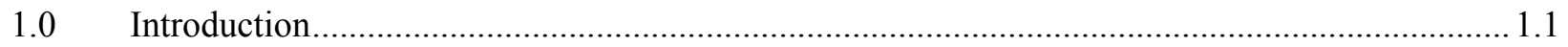

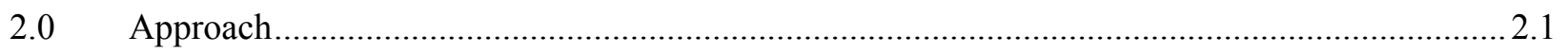

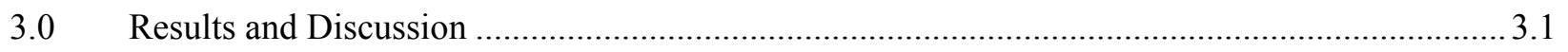

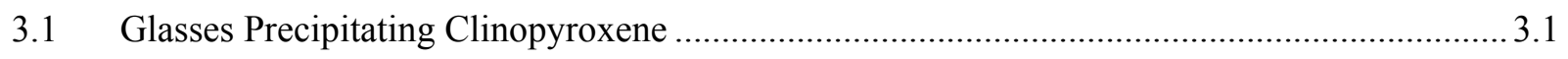

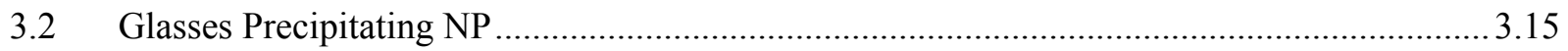

3.3 Glasses Precipitating other Crystalline Phases ................................................................. 3.31

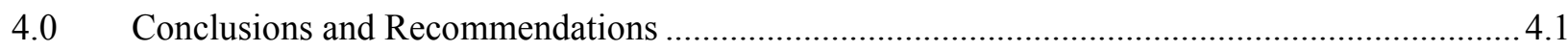

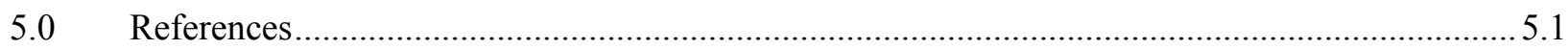

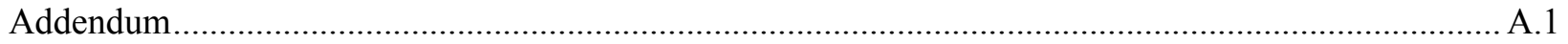

\section{Figures}

Figure 1-1. $\ln r_{B}$ from CCC Versus Quenched Glasses Within Hanford Composition Region ................ 1.3

Figure 1-2. The Effect of IHT Time on $r_{B}$ from DWPF Glass at Different Temperatures ....................... 1.5

Figure 1-3. Measured and Calculated $r_{B}$ and $r_{N a}$ from Glasses that Precipitated NP During

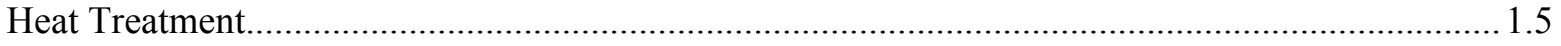

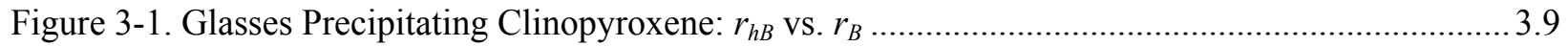

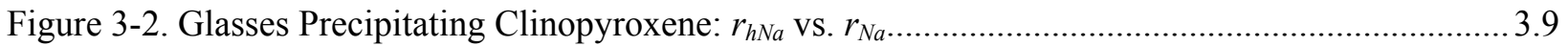

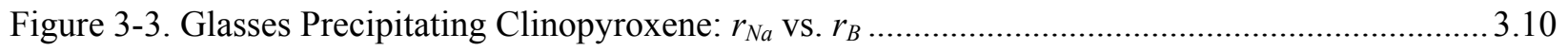

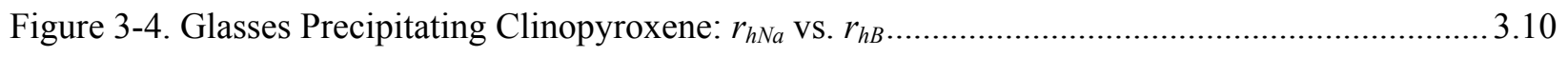

Figure 3-5. Glasses Precipitating Clinopyroxene: $r_{B}$, Calculated vs. Measured ................................... 3.11

Figure 3-6. Glasses Precipitating Clinopyroxene: $r_{N a}$, Calculated vs. Measured...................................3.11

Figure 3-7. Glasses Precipitating Clinopyroxene: $r_{r B}$ (calculated) vs. $r_{h B}$ (measured) .......................... 3.12

Figure 3-8. Glasses Precipitating Clinopyroxene: $r_{r N a}$ (calculated) vs. $r_{h N a}$ (measured) ........................ 3.13 
Figure 3-9. Glasses Precipitating Clinopyroxene: $r_{r B}$ (calculated using measured $r_{B}$ ) vs. $r_{h B}$ (measured)

Figure 3-10. Glasses Precipitating Clinopyroxene: $r_{r N a}$ (calculated using measured $r_{N a}$ ) vs. $r_{h N a}$ (measured)

Figure 3-11. Glasses Precipitating NP: $r_{h B}$ vs. $r_{B}$

Figure 3-12. Glasses Precipitating NP: $r_{h N a}$ vs. $r_{N a}$

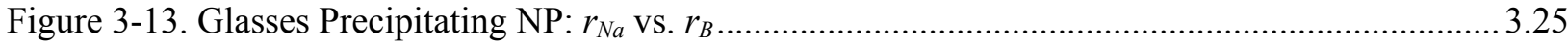

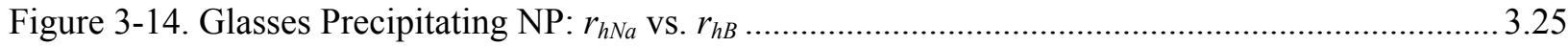

Figure 3-15. Glasses Precipitating NP: $r_{B}$, Calculated vs. Measured..................................................2.26

Figure 3-16. Glasses Precipitating NP: $r_{N a}$, Calculated vs. Measured ................................................ 3.26

Figure 3-17. Glasses Precipitating NP: $r_{r B}$ (calculated) vs. $r_{h B}$ (measured) …......................................2.27

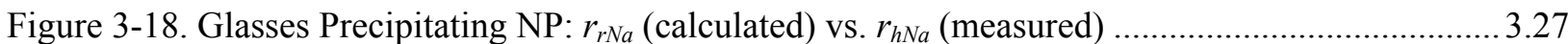

Figure 3-19. Glasses Precipitating NP: $r_{r B}$ (calculated using measured $r_{B}$ ) vs. $r_{h B}$ (measured) ..............3.29

Figure 3-20. Glasses Precipitating NP: $r_{r N a}\left(\right.$ calculated using measured $\left.r_{N a}\right)$ vs. $r_{h N a}$ (measured) ..........3.29

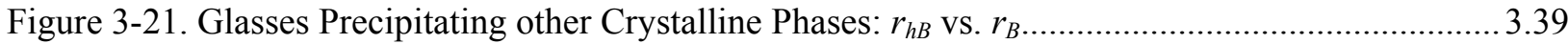

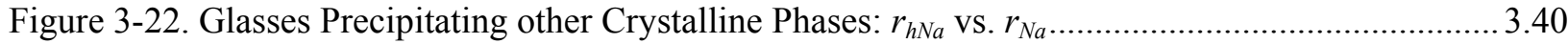

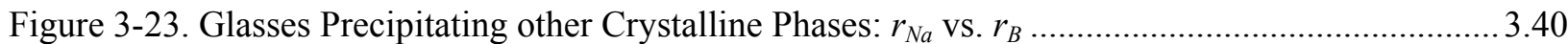

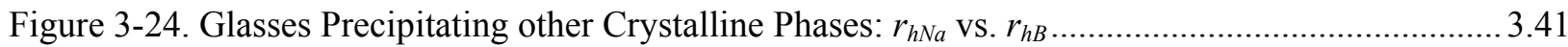

Figure 3-25. Glasses Precipitating other Crystalline Phases: $r_{B}$, Calculated vs. Measured .....................3.41

Figure 3-26. Glasses Precipitating other Crystalline Phases: $r_{N a}$, Calculated vs. Measured ....................3.42

Figure 3-27. Glasses Precipitating other Crystalline Phases: $r_{r B}$ (calculated) vs. $r_{h B}$ (measured) ............ 3.42

Figure 3-28. Glasses Precipitating other Crystalline Phases: $r_{r N a}$ (calculated) vs. $r_{h N a}$ (measured) ......... 3.44

Figure 3-29. Glasses Precipitating other Crystalline Phases: $r_{r B}$ (calculated using measured $r_{B}$ ) vs. $r_{h B}$ (measured)

Figure 3-30. Glasses Precipitating other Crystalline Phases: $r_{r N a}$ (calculated using measured $r_{N a}$ ) vs. $r_{h N a}($ measured $)$ 


\section{Tables}

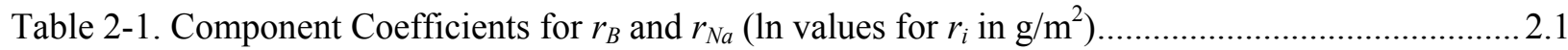

Table 2-2. HLW Glass Composition Region (in mass factions of oxides) ............................................2.1

Table 2-3. Minerals and their Effects on $r_{B}$ and $r_{N a}\left(\ln\right.$ values for $r_{i}$ in $\left.\mathrm{g} / \mathrm{m}^{2}\right)$ from the Glass .................2.2

Table 3-1. Composition of Glasses Precipitating Clinopyroxene (in mass fractions of oxides)................3.3

Table 3-2. Crystallinity (in mass fractions of minerals) of Heat-Treated Glasses with Clinopyroxene ... 3.4

Table 3-3. Residual Glass Composition (in mass fractions of oxides): Glasses Precipitating

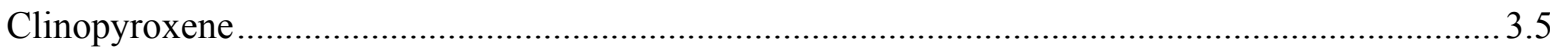

Table 3-4. Measured $r_{B}$ and $r_{N a}$ (in $\mathrm{g} / \mathrm{m}^{2}$ ) from Quenched and Heat-Treated Glasses with Clinopyroxene

Table 3-5. Calculated $r_{B}$ and $r_{N a}$ (in $\mathrm{g} / \mathrm{m}^{2}$ ) from Quenched and Heat-Treated Glasses with Clinopyroxene

Table 3-6. Composition of Glasses Precipitating NP (in mass fractions of oxides) .................................18

Table 3-7. Crystallinity (in mass fractions of minerals) of Heat-Treated Glasses with NP .................... 3.19

Table 3-8. Residual Glass Composition (in mass fractions of oxides): Glasses Precipitating NP 1.......3.20

Table 3-9. Residual Glass Composition (in mass fractions of oxides): Glasses Precipitating NP 2....... 3.21

Table 3-10. Measured $r_{B}$ and $r_{N a}\left(\right.$ in $\mathrm{g} / \mathrm{m}^{2}$ ) from Quenched and Heat-Treated Glasses with NP........... 3.22

Table 3-11. Calculated $r_{B}$ and $r_{N a}$ (in $\mathrm{g} / \mathrm{m}^{2}$ ) from Quenched and Heat-Treated Glasses with NP .......... 3.23

Table 3-12. Composition of Glasses Precipitating Lithium-Silicates .....................................................3.32

Table 3-13. Composition of Glasses Precipitating Cristobalite ...........................................................3.33

Table 3-14. Composition of Glasses Precipitating Hematite ..................................................................3.33

Table 3-15. Composition of Glasses Precipitating Zircon ....................................................................3.33

Table 3-16. Crystallinity (in mass fractions of minerals) of Heat-Treated Glasses with

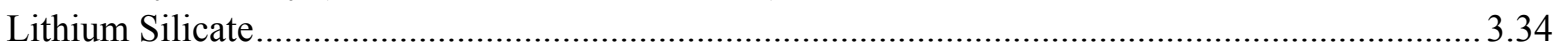

Table 3-17. Crystallinity (in mass fractions of minerals) of Heat-Treated Glasses with Cristobalite ..... 3.34

Table 3-18. Crystallinity (in mass fractions of minerals) of Heat-Treated Glasses with Hematite ..........3.34

Table 3-19. Crystallinity (in mass fractions of minerals) of Heat-Treated Glasses with Zircon ............. 3.35

Table 3-20. Residual Glass Composition (in mass fractions of oxides): Glasses Precipitating

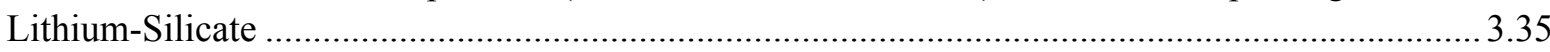

Table 3-21. Residual Glass Composition (in mass fractions of oxides): Glasses Precipitating Cristobalite..... 
Table 3-22. Residual Glass Composition (in mass fractions of oxides): Glasses Precipitating

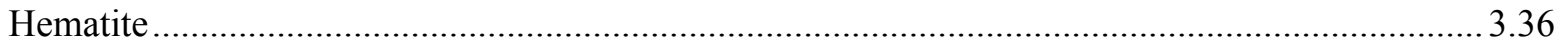

Table 3-23. Residual Glass Composition (in mass fractions of oxides): Glasses Precipitating Zircon ... 3.36

Table 3-24. Measured $r_{B}$ and $r_{N a}$ (in $\mathrm{g} / \mathrm{m}^{2}$ ) from Quenched and Heat-Treated Glasses with Lithium-Silicate 3.36

Table 3-25. Measured $r_{B}$ and $r_{N a}$ (in $\mathrm{g} / \mathrm{m}^{2}$ ) from Quenched and Heat-Treated Glasses with Cristobalite

Table 3-26. Measured $r_{B}$ and $r_{N a}$ (in $\mathrm{g} / \mathrm{m}^{2}$ ) from Quenched and Heat-Treated Glasses with Hematite .. 3.37

Table 3-27. Measured $r_{B}$ and $r_{N a}$ (in $\mathrm{g} / \mathrm{m}^{2}$ ) from Quenched and Heat-Treated Glasses with Zircon .......3.37

Table 3-28. Calculated $r_{B}$ and $r_{N a}\left(\right.$ in $\mathrm{g} / \mathrm{m}^{2}$ ) from Quenched and Heat-Treated Glasses with Lithium Silicate.

Table 3-29. Calculated $r_{B}$ and $r_{N a}$ (in $\mathrm{g} / \mathrm{m}^{2}$ ) from Quenched and Heat-Treated Glasses with Cristobalite

Table 3-30. Calculated $r_{B}$ and $r_{N a}$ (in $\mathrm{g} / \mathrm{m}^{2}$ ) from Quenched and Heat-Treated Glasses with Hematite.3.39

Table 3-31. Calculated $r_{B}$ and $r_{N a}$ (in $\mathrm{g} / \mathrm{m}^{2}$ ) from Quenched and Heat-Treated Glasses with Zircon ......3.39 


\subsection{Introduction}

The Hanford Site's original mission was to produce nuclear materials for the U.S. Department of Energy (DOE) and its predecessors. An enormous inventory of radioactive and mixed waste, largely generated during plutonium production, is stored in 177 underground single- and double-shell tanks. Under Hanford's current cleanup mission, these wastes will be retrieved and separated into low-activity waste (LAW) and high-level waste (HLW) fractions, which will be permanently stored in underground repositories. Both LAW and HLW will be vitrified.

The water-insoluble sludge, retrieved as HLW, contains components that tend to precipitate from glass during cooling. As the glass is poured into a canister, crystallization takes place within the temperature interval between the liquidus temperature $\left(T_{L}\right)$ and glass-transition temperature $\left(T_{g}\right)$. A portion of the glass cast into canisters is quenched on the canister walls, and another portion of glass, near the canister centerline, cools slowly. Thus, the temperature history of the canister centerline cooled (CCC) glass is most favorable for crystalline phases to form.

Above $T_{g}$, the average CCC rate is approximately $0.05 \mathrm{~K} / \mathrm{s}$ (Edwards 1987; Lee 1989; Casler and Hrma 1999). This cooling rate allows sufficient time for fast-nucleating and fast-growing phases, such as spinel and nepheline (NP), to precipitate. Slow-growing phases or those that do not easily nucleate, such as acmite or zircon, are less likely to form crystals in the canister (Hrma et al. 1994). As a new phase precipitates, it affects the glass matrix, in which it is embedded, both chemically and mechanically. These changes may impact the rate of glass dissolution in water and thus change its chemical durability (Kim et al. 1995; Cicero et al. 1993). This is a concern because water may eventually penetrate the repositories and contact the glass canisters after they cool below the boiling point of water. Consequently, the product consistency test (PCT) responses of glasses quenched on steel plates and those heat treated according to a simulated CCC schedule are expected to bracket the range of responses expected of all canistered glass. ${ }^{\text {a }}$

The acceptability criterion for the HLW glass repository is based on the PCT (ASTM 1998): the $r_{B}$, $r_{N a}$, and $r_{L i}$ from the glass must be lower than the $r_{B}, r_{N a}$, and $r_{L i}$ from Defense Waste Processing FacilityEnvironmental Assessment (DWPF-EA) glass (Jantzen et al. 1993). Both quenched and canister centerline glass must meet this constraint. ${ }^{\mathrm{b}}$ To formulate glasses that satisfy the acceptability criterion, constitutive equations have been developed that relate $r_{B}, r_{N a}$, and $r_{L i}$ to glass composition. For the quenched glass, it is sufficient to use first-order equations (Hrma et al. 1994) with coefficients that are

\footnotetext{
${ }^{\text {a }}$ This sentence does not suggest that PCT predicts long-term behavior of glass in a repository. Rather, it states that if PCT constraints are met for both quenched and CCC glass, it is highly probable that all glass in a canister meets the PCT constraints.

${ }^{b}$ This is a minimum requirement. The uncertainties in the canister-centerline cooling rate, the spatial nonuniformity of crystallinity, and the assumption that PCT releases are monotonous functions of the position vector of canistered glass have not been established.
} 
estimated from measured data. Several sets of such coefficients have been reported for different HLW glass-composition regions (Hrma et al. 1994, Hrma et al. 1995).

The effect of CCC on the PCT of HLW glasses was determined for more than 100 glass compositions (Hrma et al. 1994; Hrma et al. 1995; Kim et al. 1995), as shown in Figure 1-1. The $r_{B}$ s from CCC and quenched glasses were close in value for most of the compositions regardless of the fraction of crystallinity that formed during heat treatment. For only 18 of these glasses, CCC treatment significantly increased the $r_{B}$ as compared to quenched glasses. Six compositions exhibited a moderate increase in $r_{B}$ from CCC glass, and twelve CCC-treated compositions showed a strong increase in $r_{B}$. Those glasses with a moderate increase in $r_{B}$ contained zircon, clinopyroxene, and/or hematite crystals while those with a strong increase in $r_{B}$ contained alkali aluminosilicates (NP and eucryptite) and cristobalite. Cristobalite could be produced by the crystallization of $\mathrm{a} \mathrm{SiO}_{2}$-rich immiscible liquid during cooling. Surprisingly, the $r_{B}$ of three glasses that remained crystal-free after the CCC-treatment increased by an order of magnitude. No evidence of phase separation was found in these glasses. So far, no attempt was made to elucidate the cause of the increase in $r_{B}$ for these glasses.

Attempts to relate $r_{B}$ and $r_{N a}$ to glass composition via first- or second-order polynomial functions yielded good results for large composition regions of quenched HLW glasses, but were relatively unsuccessful for CCC-treated glasses. When the fraction and composition of the crystalline phase was known, measured $r_{B}$ s were in good agreement with those calculated for the composition of the residual glass (Kim et al. 1995; Bailey and Hrma 1995; Hrma and Bailey 1995; Li et al. 1997). A study of high waste-loaded glasses (Bailey and Hrma 1995) indicated that the residual glass composition was the major factor that controlled the PCT response of glasses with durable crystalline phases. ${ }^{\text {a }}$ Other chemical or mechanical factors, such as concentration gradients and mechanical stresses, played a secondary role.

\footnotetext{
${ }^{a}$ This report does not address the issues related to glasses with a major fraction of crystallinity. Such glasses promise increased waste loading. However, they present challenges to processing technology. Also, radionuclide partitioning will need to be considered as a waste-form performance assessment issue.
} 


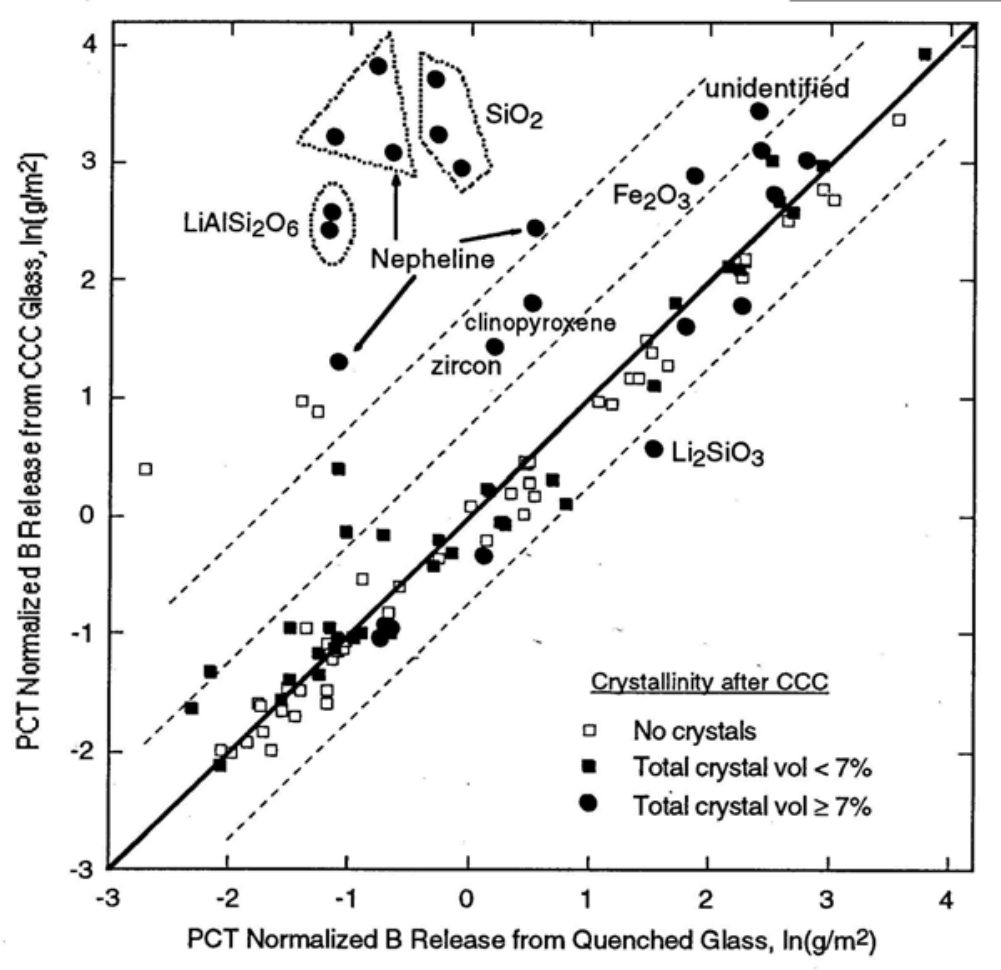

\section{Figure 1-1. In $r_{B}$ from CCC Versus Quenched Glasses Within Hanford Composition Region \\ (Kim et al. 1995)}

An example of the effect of isothermal heat treatment on $r_{B}$ [Cicero et al. 1993], is shown in Figure 1-2. The $r_{B}$ slightly decreased with prolonged heat treatment at $\mathrm{T} \geq 900^{\circ} \mathrm{C}$, presumably because of alkaline and boron volatilization (these components decrease glass durability). At $900^{\circ} \mathrm{C}, r_{B}$ increased slightly with time at $\mathrm{t}>10 \mathrm{~h}$, and increased by an order of magnitude at $600^{\circ} \mathrm{C}$ and $700^{\circ} \mathrm{C}$. This increase in $\mathrm{B}$ release was accompanied by precipitation of crystalline phases from the glass.

Figure 1-3 compares measured and calculated $r_{B}$ and $r_{N a}$ for data reported by Li et al. (1997). The measured $r_{B}$ and $r_{N a}$ for these glasses were higher than predicted. The calculated curves represent $r_{B}$, calculated for the residual glass compositions using a first-order model, with coefficients determined from quenched glass data. The residual glass composition was obtained from a mass balance written for stoichiometric NP $\left(\mathrm{NaAlSiO}_{4}\right)$. The measured fractions of NP were determined with a semiquantitative $\mathrm{x}$ ray diffraction (XRD) method. The calculated fractions of NP to produce the measured releases were higher by several mass\% than the actual values determined by XRD. This discrepancy cannot be attributed to the silica excess in NP (Menkhause et al. 2000) because the effect of excess silica is compensated by a lower concentration of $\mathrm{Na}_{2} \mathrm{O}$. The cause of this discrepancy is unclear. One possible explanation is that the residual glass composition was outside the composition region on which the PCT 
models were valid (these models were obtained by fitting model equations to quenched-glass PCT databases).

These studies indicate that it may be possible to estimate PCT releases from CCC glass on the basis of the composition of the residual glass. This report tests this hypothesis using the database of 212 heattreated (mostly CCC) glasses, for which both the crystalline phases, as determined with semiquantitative $\mathrm{XRD}$, and PCT releases are documented. It summarizes data and evaluates the effect of CCC on $r_{B}$ and $r_{N a}$ for major PCT-impacting crystalline phases. As discussed in the test plan (Hrma et al., 2000), the ultimate goal is to understand the mechanism of this effect and to develop relationships between $r_{B}$ and $r_{\mathrm{Na}}$ from CCC glasses and glass composition. In this report, our main focus is to develop and verify a functional relationship between $r_{B}$ and $r_{N a}$ from heat-treated glasses and residual glass composition using previously published data. We grouped glasses according to major crystalline phases that precipitated upon heat treatment. For each heat-treated glass, we calculated residual glass composition and estimated PCT releases. The results are compared with measured values.

In this report, we describe the approach taken in this research and three general types of glasses that were examined: those that precipitate clinopyroxene, those that precipitate NP, and those that precipitate other crystalline phases. Finally, we present conclusions and recommendations. 


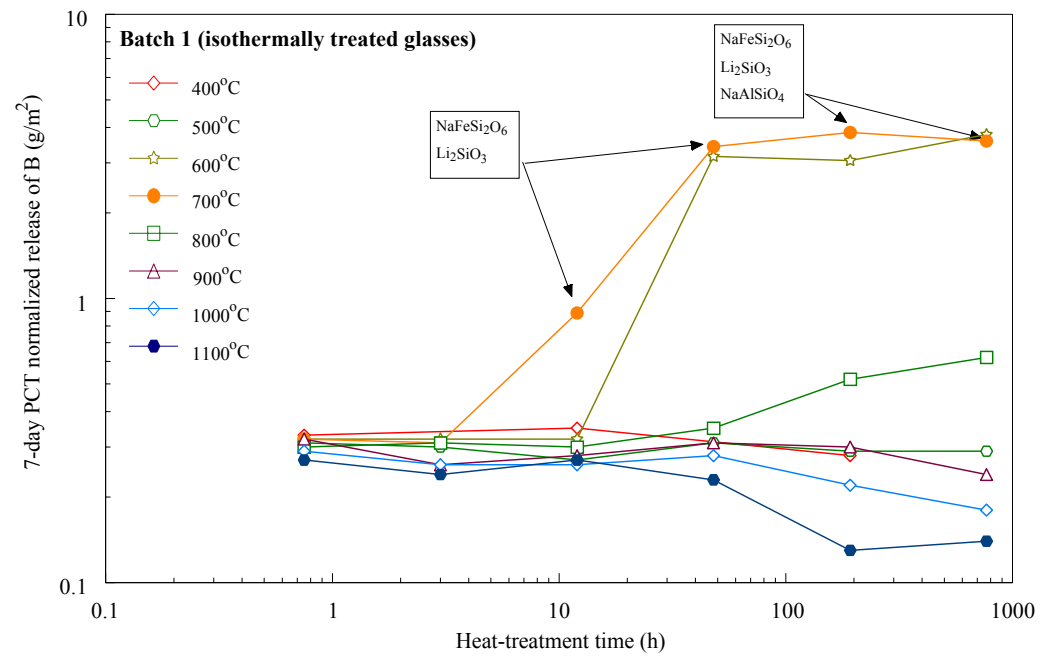

\section{Figure 1-2. The Effect of IHT Time on $r_{B}$ from DWPF Glass at Different Temperatures (Cicero et al. 1993) (a)}

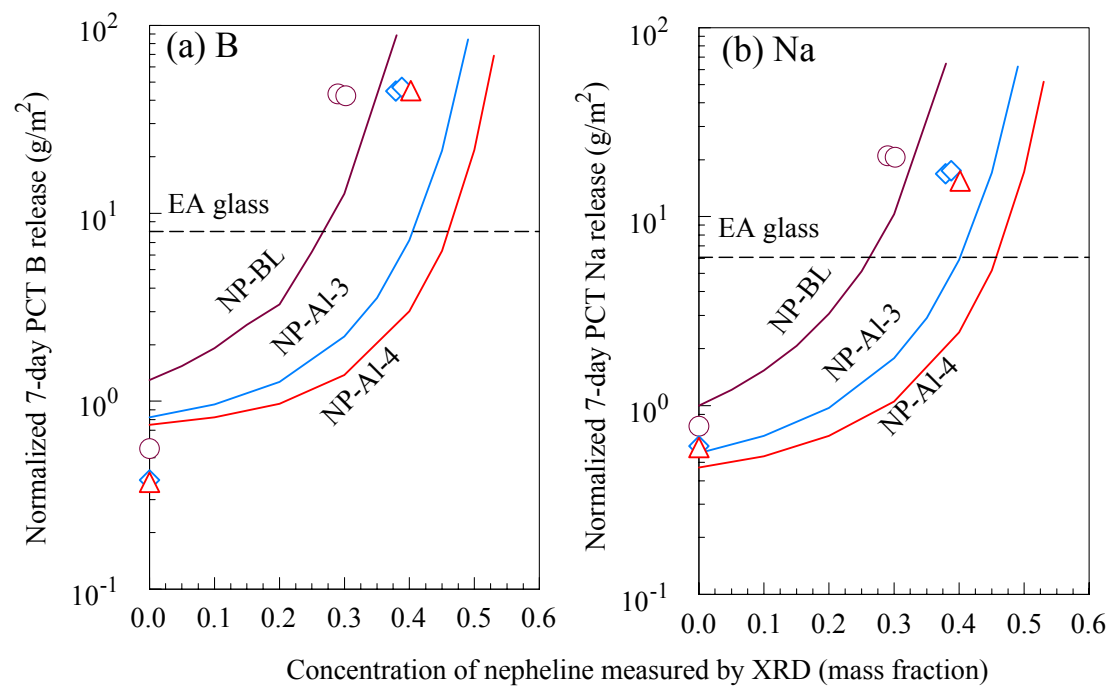

\section{Figure 1-3. Measured and Calculated $r_{B}$ and $r_{N a}$ from Glasses that Precipitated NP \\ During Heat Treatment}

(a) H. Li generated this plot from data by Cicero et al. (1993). 


\subsection{Approach}

A large number of glasses were prepared at the Pacific Northwest National Laboratory (PNNL) and the Savannah River Technology Center (SRTC). The compositions of these glasses and their properties, including $r_{B}$ and $r_{N a}$ from quenched as well as heat-treated samples, were reported in several papers (Hrma et al. 1994; Vienna et al. 1996; Cicero et al. 1993; Marra and Jentzen 1993). For the quenched glass, it is sufficient to use the equation (Hrma et al. 1994)

$$
\ln r_{j}=\sum_{i=1}^{N} b_{i j} g_{i}
$$

where, $g_{i}$ is the $i$-th glass component mass fraction, $b_{i j}$ is the $i$-th component coefficient for $j$-th element release, and $N$ is the number of components.

Component coefficients (the $b_{i j}$ values) for $r_{j}(j=\mathrm{B}, \mathrm{Na}, \mathrm{Li}$, and $\mathrm{Si}$ ) from quenched glasses were developed by fitting first-order polynomials to PNNL data (Hrma, et al. 1994; Hrma, et al. 1995) and are listed for $r_{B}$ and $r_{N a}$ in Table 2.1. Similar treatment can be used with $b_{i j}$ values reported by Jantzen (1992). The validity of the component coefficients listed in Table 2.1 is restricted to the glass-composition region shown in Table 2.2.

Table 2.1. Component Coefficients for $r_{B}$ and $r_{N a}$ (ln values for $r_{j}$ in $\left.\mathrm{g} / \mathrm{m}^{2}\right)$

\begin{tabular}{|c|c|c|c|c|c|c|c|c|c|c|c||}
\hline \hline & $\mathbf{A l}_{2} \mathbf{O}_{3}$ & $\mathbf{B}_{2} \mathbf{O}_{\mathbf{3}}$ & $\mathbf{C a O}$ & $\mathbf{F e}_{2} \mathbf{O}_{3}$ & $\mathbf{L i}_{2} \mathbf{O}$ & $\mathbf{M g O}$ & $\mathbf{N a}_{2} \mathbf{O}$ & $\mathbf{S i O}_{2}$ & $\mathbf{Z r O}_{2}$ & Others \\
\hline $\mathrm{b}_{\mathrm{iB}}$ & -25.41 & 12 & -8.71 & -3.2 & 22.6 & 10.9 & 17.6 & -4.32 & -10.56 & 0.16 \\
\hline $\mathrm{b}_{\mathrm{iNa}}$ & -25.43 & 9.41 & -1.96 & -4.1 & 19.1 & 11.8 & 19.4 & -4.41 & -11.42 & -0.66 \\
\hline \hline
\end{tabular}

Table 2.2. HLW Glass Composition Region (in mass factions of oxides)

\begin{tabular}{||l|c|c||}
\hline \hline Components & Minimum & Maximum \\
\hline $\mathrm{Al}_{2} \mathrm{O}_{3}$ & 0.000 & 0.170 \\
\hline $\mathrm{B}_{2} \mathrm{O}_{3}$ & 0.050 & 0.200 \\
\hline $\mathrm{CaO}$ & 0.000 & 0.100 \\
\hline $\mathrm{Fe}_{2} \mathrm{O}_{3}$ & 0.005 & 0.150 \\
\hline $\mathrm{Li}_{2} \mathrm{O}$ & 0.010 & 0.070 \\
\hline $\mathrm{MgO}$ & 0.000 & 0.080 \\
\hline $\mathrm{Na}_{2} \mathrm{O}$ & 0.050 & 0.200 \\
\hline $\mathrm{SiO}_{2}$ & 0.420 & 0.570 \\
\hline $\mathrm{ZrO}_{2}$ & 0.000 & 0.130 \\
\hline Others & 0.010 & 0.100 \\
\hline
\end{tabular}


Crystalline phases identified in glasses by XRD are listed in Table 2.3 (minerals with $c_{k}<0.005$ were generally not detected; the error could be as high as $\pm 50 \%$ ). Each of these phases may contain a large number of elements. With exception of NP, only idealized chemical formulas are shown in Table 2.3, including mass fractions of individual oxides calculated from the chemical formulas and the expression

$$
\ln r_{c j k}=\sum_{i=1}^{K} b_{i j} c_{i k}
$$

where $r_{c j k}$ is the equivalent $j$-th element PCT normalized release from the $k$-th mineral (the extent to which $k$-th mineral precipitation impacts the $r_{j}$ from heat-treated glass), $c_{i k}$ is the $i$-th oxide mass fraction in the $k$-th mineral, and $K$ is the number of oxides in the mineral. Roughly, if $r_{c j k}$ value is larger than $2 \mathrm{~g} / \mathrm{m}^{2}$, a the precipitation of the $k$-th mineral will decrease $r_{j}$ of the glass. As long as the crystals are fully embedded in glass, their durability does not significantly affect the PCT response of the heat-treated glass.

Table 2.3. Minerals and their Effects on $r_{B}$ and $r_{N a}$ (In values for $r_{j}$ in $\mathrm{g} / \mathrm{m}^{2}$ ) from the Glass

\begin{tabular}{|c|c|c|c|c|c|c|c|c|c|c|c|c|}
\hline Mineral & Formula & $\mathbf{A l}_{2} \mathbf{O}_{3}$ & $\mathrm{CaO}$ & $\mathrm{Fe}_{2} \mathrm{O}_{3}$ & $\mathbf{L i}_{2} \mathbf{O}$ & MgO & $\mathrm{Na}_{2} \mathbf{O}$ & $\mathrm{SiO}_{2}$ & $\mathrm{ZrO}_{2}$ & Others & $\ln r_{\mathrm{cBk}}$ & In $\mathbf{r}_{\mathrm{cNak}}$ \\
\hline Acmite $^{(b)}$ & $\mathrm{NaFeSi}_{2} \mathrm{O}_{6}$ & & & 0.346 & & & 0.134 & 0.520 & & & -1 & -1.1 \\
\hline Albite & $\mathrm{NaAlSi}_{3} \mathrm{O}_{8}$ & 0.194 & & & & & 0.118 & 0.687 & & & -5.8 & -5.7 \\
\hline Cristobalite & $\mathrm{SiO}_{2}$ & & & & & & & 1 & & & -4.3 & -4.4 \\
\hline Enstatite $^{(\mathrm{c})}$ & $\mathrm{MgSiO}_{3}$ & & & & & 0.401 & & 0.599 & & & 1.8 & 2.1 \\
\hline Eucryptite & $\mathrm{LiAlSi}_{3} \mathrm{O}_{8}$ & 0.207 & & & 0.061 & & & 0.732 & & & -7.1 & -7.3 \\
\hline Gehlinite & $\mathrm{Ca}_{2} \mathrm{Al}_{2} \mathrm{SiO}_{7}$ & 0.372 & 0.409 & & & & & 0.219 & & & -14 & -11.2 \\
\hline Hematite & $\mathrm{Fe}_{2} \mathrm{O}_{3}$ & & & 1 & & & & & & & -3.2 & -4.1 \\
\hline Lithium silicate & $\mathrm{Li}_{2} \mathrm{SiO}_{3}$ & & & & 0.332 & & & 0.668 & & & 4.6 & 3.4 \\
\hline $\mathrm{NP} 1^{(\mathrm{d})}$ & $\mathrm{NaAlSiO}_{4}$ & \begin{tabular}{|l|}
0.359 \\
\end{tabular} & & & & & 0.218 & 0.423 & & & -7.1 & -6.8 \\
\hline $\mathrm{NP} 2^{(\mathrm{e})}$ & & \begin{tabular}{|l|}
0.282 \\
\end{tabular} & & 0.070 & & & 0.144 & 0.504 & & & -7.0 & -6.9 \\
\hline Olivine & $\mathrm{Mg}_{2} \mathrm{SiO}_{4}$ & & & & & 0.573 & & 0.427 & & & 4.4 & 4.9 \\
\hline Trevorite $^{(\mathrm{a})}$ & $\mathrm{NiFe}_{2} \mathrm{O}_{4}$ & & & 0.681 & & & & & & 0.319 & -2.1 & -3 \\
\hline Wollastonite & $\mathrm{CaSiO}_{3}$ & & 0.483 & & & & & 0.517 & & & -6.4 & -3.2 \\
\hline Zircon & $\mathrm{ZrSiO}_{4}$ & & & & & & & 0.397 & 0.603 & & -8.1 & -8.6 \\
\hline
\end{tabular}

(a) Trevorite represents spinel

(b) Acmite represents clinopyroxene

${ }^{(c)}$ Enstatite represents orthopyroxene

(d)NP 1 represents an idealized composition

${ }^{(\mathrm{e})} \mathrm{NP} 2$ represents measured NP composition (Menkhaus et al. 2000)

${ }^{a}$ This number is based on the experience with formulating and developing HLW glasses for Hanford and INEEL. 
The residual glass composition is given by the formula (Kim et al. 1995; Hrma and Bailey 1995)

$$
g_{r i}=\frac{g_{i}-\sum_{k=1}^{M} c_{k} c_{i k}}{1-\sum_{k=1}^{M} c_{k}}
$$

where $g_{r i}$ is the $i$-th component fraction in residual glass, $c_{i k}$ is the $i$-th component fraction in the crystalline phase, $c_{k}$ is the $k$-th crystalline phase fraction in glass, and $M$ is the amount of crystal phases present in the glass. Rewriting Equation (1) for residual glass,

$$
\ln r_{r j}=\sum_{i=1}^{N} b_{i j} g_{r i}
$$

where $r_{r j}$ is $j$-th element normalized PCT release from residual glass; then using Equation (2), we obtain

$$
\ln r_{r j}=\frac{\ln r_{j}-\sum_{k=1}^{M} c_{k} \ln r_{c j k}}{1-\sum_{k=1}^{M} c_{k}}
$$

Here $r_{j}$ is the $j$-th element PCT normalized release from quenched glass.

According to Equation (5), minerals with large negative values of $\ln r_{c j k}$ are likely to significantly impact the $r_{r j}$ provided their concentration is sufficiently high. Thus ghelenite, with $\ln r_{c B k}$ and $\ln r_{c N a k}$ values of -14 and -11 (Table 2.3), has the largest impact; fortunately, this mineral, which was observed in a high-CaO experimental glass, is not likely to occur in Hanford and Savannah River HLW glasses. The next highest impact is predicted for baddeleyte $\left(\ln r_{c B k}=-11\right)$ and zircon $\left(\ln r_{c B k}=-8\right)$. Fortunately, large fractions of baddeleyite or zircon precipitated during CCC in only a few glasses in our database.

However, aluminosilicates (NP and eucryptite), with $\ln r_{c B k}=-7$, are prone to massive precipitation (up to 40 mass\%, see Section 3.2), and thus are the most insidious crystalline phases to occur in HLW glass. Albite with $\ln r_{c B k}=-6$ usually occurs in a solid solution with NP. NP 2 is such a solid solution, also containing $\mathrm{Fe}_{2} \mathrm{O}_{3}$. The impact of NP 2 on $r_{i}$ is roughly the same as that of stoichiometric NP. Other phases with negative values of $\ln r_{c j k}$ are cristobalite, hematite, wollastonite, and trevorite. Of this group, only trevorite is likely to occur in HLW glass. ${ }^{\text {a }}$ Trevorite is a common crystalline phase, but its concentration is typically $<1$ mass\% in CCC-treated HLW glasses, and thus its impact on PCT response is negligible.

\footnotetext{
${ }^{a}$ It is assumed that glasses are formulated to avoid phase separation that can lead to precipitation of cristobalite.
} 
Disregarding olivine, a mineral that is unlikely to occur in HLW glass, the only crystalline phase with a positive $r_{c B k}$ is lithium-silicate (with $\ln r_{c B k}=5$ ). Acmite, the major clinopyroxene phase with $\ln r_{c B k}=1$, has virtually no impact on $r_{j}$ of HLW glasses.

In the following sections, we will divide existing data into three groups according to the major crystalline phase that precipitates during heat treatment, either CCC or isothermal heat treatment (IHT). The major crystalline phases are clinopyroxene, NP, and others (lithium silicates, cristobalite, hematite, and zircon). We did not consider spinel as a major component because of its low concentration in HLW glass. We included clinopyroxene in the analysis, despite its small impact on $r_{B}$ and $r_{N a}$, to investigate whether other factors, such as residual stresses, impact PCT. 


\subsection{Results and Discussion}

\subsection{Glasses Precipitating Clinopyroxene}

A typical mineral representing the clinopyroxene family is acmite, $\mathrm{NaFeSi}_{2} \mathrm{O}_{6}$. The clinopyroxene phase that precipitates from HLW glass is a solid solution of acmite, the major component, and other clinopyroxenes. Scanning electron microscopy-energy dispersive spectroscopy (SEM-EDS) shows the presence of $\mathrm{Ca}, \mathrm{Mg}, \mathrm{Ni}$, and $\mathrm{Cr}$ in the clinopyroxenes found in typical HLW glasses (Hrma et al. 1994, Plaisted et al. 2001). In this report, we ignore the minor components and consider all clinopyroxene to be of the acmite composition. As Table 2.3 shows, acmite has a hardly detectable (predicted) effect on $r_{B}$ and $r_{N a}$, indicating that secondary effects, such as those of internal stresses, micro-cracks, or concentration gradients associated with the precipitation of clinopyroxene have little impact on $r_{B}$ and $r_{N a}$. Table 3.1 lists compositions of glasses that precipitated clinopyroxene upon heat treatment, CCC, or IHT. Note that composition variability study (CVS) glasses were heat-treated using CCC (see Hrma et al. 1994); the remaining compositions underwent IHT under conditions described in Cicero et al. (1993); see Section 1.0 .

Table 3.2 summarizes mass fractions of crystalline phases after the heat treatments. Most glasses precipitated more than one crystalline phase. Two glasses included in this group had zircon as a major crystalline phase and four glasses precipitated substantial fractions of lithium-silicate. After heat treatment, the concentrations of crystalline phases were determined with semi-quantitative XRD. The residual glass compositions, obtained from Equation (2), including all crystalline phases detected, are listed in Table 3.3. Comparison with Table 2.2 shows that all residual-glass compositions but one are within the validity limits for $r_{B}$ and $r_{N a}$ coefficients listed in Table 2.1. Only the $\mathrm{Fe}_{2} \mathrm{O}_{3}$ fraction in CVS1-8 glass exceeds the upper limit by 1.7 mass $\%$.

Measured $r_{B}$ and $r_{N a}$ from quenched and heat-treated glasses are summarized in Table 3.4 (in the remainder of this report, we occasionally drop, for convenience, subscripts $h$ and $r$ from $r_{r j}$ and $r_{h j}[j=\mathrm{B}$, $\mathrm{Na}]$ and denote the corresponding releases as $r_{B}$ and $r_{N a}$ from heat-treated glasses, calculated $\left[r_{r j}\right]$ or measured $\left[r_{h j}\right]$, when the meaning of symbols is clear from the context). The $r_{h B}$ and $r_{h N a}\left(r_{B}\right.$ and $r_{N a}$ from heat-treated glasses) are plotted against the $r_{B}$ and $r_{N a}$ in Figure 3-1 and Figure 3-2. Except for CVS1-8, CVS1-22, CVS2-42, and CVS2-25 (see Table 3.4 and Table 3.5 ${ }^{\mathrm{a}}$ ), the releases from heat-treated and quenched glasses are identical within experimental error. ${ }^{b}$ For CVS1-8, CVS1-22, and CVS2-42, the $r_{h B}$ and $r_{h N a}$ are higher from CCC glass than $r_{B}$ and $r_{N a}$ from quenched glass. Only for CVS2-25, the $r_{h B}$ and

${ }^{a}$ Glass IDs are not indicated in the figures; the reader is advised to check the data tables for evidence.

${ }^{\mathrm{b}}$ Experimental error in data used for calculation is difficult to assess quantitatively, taking into account uncertainties of semiquantitative XRD and SEM EDS. Model uncertainty should also be considered. This uncertainty can be judged from calculated versus measured $r_{B}$ and $r_{N a}$ values for quenched glasses. This error substantially increases for compositions close to or outside the boundary of the composition region for which the models were validated. 
$r_{h N a}$ are lower from CCC glass than $r_{B}$ and $r_{N a}$ from quenched glass. Glasses CVS1-8 and CVS1-22 were the only two glasses that formed zircon (11 mass\% each) in addition to clinopyroxene. Glass CVS2-25 formed 8 mass $\%$ acmite, 8 mass $\%$ hematite, and 10 mass\% lithium-silicate. Finally, glass CVS2-42 precipitated 20 mass $\%$ acmite and 12.5 mass $\%$ lithium-silicate. These four glasses appear less resistant to the PCT $\left(r_{B}\right.$ and $\left.r_{N a}>1 \mathrm{~g} / \mathrm{m}^{2}\right)$ than the rest. An exception is glass CVS1-15 that precipitated $2.5 \mathrm{mass} \%$ lithium silicate. For this glass, with the highest $r_{B}$ and $r_{N a}$ of the whole set, CCC did not affect the release values. Figure 3-3 and Figure 3-4 compare $r_{N a}$ with $r_{B}$ for quenched glasses with $r_{h B}$ and $r_{h N a}$ from heattreated glasses. The releases are identical within experimental error for glasses with $r_{N a}$ and $r_{B}<1 \mathrm{~g} / \mathrm{m}^{2}$. For glasses with $r_{N a}$ and $r_{B}>1 \mathrm{~g} / \mathrm{m}^{2}, r_{N a}<r_{B}$ and $r_{h N a}<r_{h B}$. The differences are slight but systematic. Quenched glass $r_{N a}<r_{B}$ indicates the participation of $\mathrm{Na}$ in the secondary mineral products or absorption on such products in the PCT solution. The partitioning of Na between residual glass and clinopyroxene, a relatively more durable phase, could contribute to somewhat higher differences $\left(r_{h N a}<r_{h B}\right)$ in heat-treated samples (the measured $r_{h B}$ and $r_{h N a}$ were taken as reported; they were not renormalized with respect to the calculated composition of residual glass).

Table 3.5 summarizes calculated $r_{B}$ and $r_{N a}$. For quenched glasses, Equation (1) was used with coefficients listed in Table 2.1. The results are plotted in Figure 3-5 and Figure 3-6. The predictions are excellent for $r_{B}$ from glasses with $r_{B}$ and $r_{N a}<1 \mathrm{~g} / \mathrm{m}^{2}$. For glasses with $r_{B}$ and $r_{N a}>1 \mathrm{~g} / \mathrm{m}^{2}$, Equation (1) provides slightly underpredicted values. For most glasses with the $r_{N a}<1 \mathrm{~g} / \mathrm{m}^{2}, r_{N a}$ values are slightly overpredicted. The component coefficients were derived from the whole CVS1 and CVS2 database. Systematic deviations of predicted values from measured values, when the coefficients are applied to a certain subset of the original database, may indicate differences in glass structure or an involvement of components in aqueous reactions that occur in the solution.

Releases from heat-treated glasses were calculated using Equation (4). In this equation, the sum of terms expressing the effects of the crystalline phases is subtracted from $\ln r_{j}$. For the $r_{j}$ (quenched glass), we used both the measured and calculated values. The results are shown in Table 3.5 and plotted in Figure 3-7 to Figure 3-10. For durable glasses $\left(r_{j}<1 \mathrm{~g} / \mathrm{m}^{2}\right)$ and CVS1-15 glass, the calculated $r_{r j}$ values (heattreated glass) using the former method (measured $r_{j}$ values) are in excellent agreement with measured data (Figure 3-9 and Figure 3-10). When the calculated $r_{j}$ values were used, the agreement was not as good (Figure 3-7 and Figure 3-8).

As we have seen, four glasses were affected by CCC treatment: CVS1-8, CVS1-22, CVS2-25, and CVS2-42. With the exception of CVS2-42 glass, the predicted $r_{r j}$ values are in a reasonable agreement with the measured values. Interestingly, a better agreement resulted when calculated releases from quenched glasses were used for this glass. Glass CVS2-42, which precipitated 20 mass $\%$ acmite and 12.5 mass\% lithium-silicate under CCC, resulting in increased B and Na releases, had substantially underpredicted $r_{r j}$ values. These low predicted values result from the light effect of acmite on PCT in combination with the strong $r_{j}$-decreasing effect of lithium-silicate. Several causes of this discrepancy can be considered: exaggerated estimate of the lithium-silicate content by semi-quantitative XRD; secondary effects, such as internal stresses; crystallization-induced phase separation leading to $\mathrm{SiO}_{2}$-impoverished glass phase. 
Table 3.1. Composition of Glasses Precipitating Clinopyroxene (in mass fractions of oxides)

\begin{tabular}{|c|c|c|c|c|c|c|c|c|c|c|}
\hline Glass & $\mathrm{Al}_{3} \mathrm{O}_{2}$ & $\mathrm{~B}_{3} \mathrm{O}_{2}$ & $\mathrm{CaO}$ & $\mathrm{Fe}, \mathrm{O}_{2}$ & Li,O & MgO & $\mathrm{Na}, \mathrm{O}$ & SiO, & \begin{tabular}{|l|}
$\mathrm{ZrO}$ \\
\end{tabular} & 00 \\
\hline $\mathrm{CV}$ & 636 & 0.1142 & 0.0275 & & 0.0376 & 0363 & & & & \\
\hline & & 0500 & & & & & & & & \\
\hline CV & 000 & 500 & 000 & 200 & 00 & & & & & \\
\hline $\mathrm{CVS}$ & 636 & 42 & 275 & 568 & & & & & & 406 \\
\hline $\mathrm{CV}$ & & & & & & & & & & \\
\hline CV: & 00 & 500 & 00 & 0 & 0 & 0 & & & 00 & 100 \\
\hline CV & & & & & & & & & & \\
\hline CVs & & & & & & & & & & 249 \\
\hline CVS & 33 & 500 & 300 & 500 & 0. & 000 & & & & 566 \\
\hline $\mathrm{CV}$ & & & & & & & & & & \\
\hline $\mathrm{CVs}$ & 00 & 500 & 20 & 00 & 0 . & 30 & & 76 & 00 & 299 \\
\hline $\mathrm{CV}$ & & & & & & & & & & \\
\hline & & & & & & & & & & \\
\hline $\mathrm{CVS}$ & 36 & 42 & 275 & 568 & 0 & 363 & & & 429 & 406 \\
\hline Ble & & & & & & & & & & \\
\hline Bler & & & 03 & & & & & & 14 & \\
\hline Bler & 16 & & 03 & 991 & & & & & & \\
\hline & & & & & & & & & & \\
\hline Batc & 488 & 778 & 122 & 284 & 0. & 42 & & & 10 & 87 \\
\hline Batc & & 778 & & 284 & & & & 346 & 010 & 787 \\
\hline Batc & 63 & & & & & & & & & \\
\hline Batc & 463 & 88 & 08 & 12 & 0.0 & & & & 017 & 790 \\
\hline & & & & & & & & & & \\
\hline Batc & 463 & & 08 & 0 & & & & & & \\
\hline Batc & 344 & 769 & 0.0099 & 171 & 0.0451 & 42 & 01 & 0.5301 & 0.0012 & 810 \\
\hline Batch 3172 & 0.0344 & & 0.0099 & & & & & & & \\
\hline Batc & 0.0344 & 0.0769 & 0.0099 & 171 & 0.0451 & 0. & & 0.5301 & 012 & 810 \\
\hline Batc & 0.0344 & 0.0769 & & 0.1171 & 0.0451 & & & & & 310 \\
\hline Batch 4171 & 0.0343 & 0.0814 & 0.0084 & 0.1171 & 0.0429 & 0.0143 & 0.0916 & 15 & 022 & 63 \\
\hline Batc & 0.0343 & 0.0814 & 0.0084 & 0.1171 & 0.0429 & 0.0143 & 0.0916 & 0.5015 & 0.0022 & 0.1063 \\
\hline Batch 4272 & 0.0343 & 0.0814 & 0.0084 & 0.1171 & 0.0429 & 0.0143 & 0.0916 & 0.5015 & 0.0022 & 0.1063 \\
\hline HM 171 & 0.0715 & 0.0703 & 0.0101 & 0.0778 & 0.0462 & 0.0149 & 0.0856 & 0.5538 & 0.0033 & 0.0665 \\
\hline HM 172 & 0.0715 & 0.0703 & 0.0101 & 0.0778 & 0.0462 & 0.0149 & 0.0856 & 0.5538 & 0.0033 & 0.0665 \\
\hline HM 271 & 0.0715 & 0.0703 & 0.0101 & 0.0778 & 0.0462 & 0.0149 & 0.0856 & 0.5538 & 0.0033 & 0.0665 \\
\hline HM 272 & 0.0715 & 0.0703 & 0.0101 & 0.0778 & 0.0462 & 0.0149 & 0.0856 & 0.5538 & 0.0033 & 0.0665 \\
\hline
\end{tabular}


Table 3.2. Crystallinity (in mass fractions of minerals) of HeatTreated Glasses with Clinopyroxene

\begin{tabular}{|c|c|c|c|c|c|c|c|}
\hline Glass & Acmite & Enstatite & Hematite & Lithium-silicate & Spinel & Zircon & Sum \\
\hline CVS1-1 & 0.070 & & & & & & 0.070 \\
\hline CVS1-8 & 0.040 & 0.030 & & & & \begin{tabular}{|l|}
0.110 \\
\end{tabular} & 0.180 \\
\hline CVS1-15 & 0.020 & & & 0.025 & & & 0.060 \\
\hline CVS1-19 & 0.090 & & & & & & 0.090 \\
\hline CVS1-20 & 0.090 & & & & & & 0.090 \\
\hline CVS1-22 & 0.025 & 0.025 & & & & \begin{tabular}{|l|}
0.110 \\
\end{tabular} & 0.160 \\
\hline CVS1-23 & 0.025 & & & & & & 0.030 \\
\hline CVS2-2 & 0.050 & & & & & & 0.050 \\
\hline CVS2-25 & 0.080 & & 0.040 & 0.100 & & & 0.220 \\
\hline CVS2-36 & 0.050 & & & 0.020 & & & 0.070 \\
\hline CVS2-42 & 0.200 & & & 0.125 & & & 0.330 \\
\hline CVS2-50 & 0.120 & & & & & & 0.120 \\
\hline CVS2-71 & 0.030 & 0.010 & & & & & 0.040 \\
\hline CVS2-96 & 0.150 & & & & & & 0.150 \\
\hline Blend 1171 & 0.009 & & & & 0.016 & & 0.030 \\
\hline Blend 1172 & 0.007 & & & & 0.015 & & 0.020 \\
\hline Blend 1272 & 0.008 & & & & 0.019 & & 0.030 \\
\hline Batch 1171 & 0.010 & & & & 0.016 & & 0.030 \\
\hline Batch 1271 & 0.008 & & & & \begin{tabular}{|l|}
0.016 \\
\end{tabular} & & 0.020 \\
\hline Batch 1272 & 0.008 & & & & 0.016 & & 0.020 \\
\hline Batch 2171 & 0.009 & & & & 0.017 & & 0.030 \\
\hline Batch 2172 & 0.011 & & & & \begin{tabular}{|l|}
0.019 \\
\end{tabular} & & 0.030 \\
\hline Batch 2271 & 0.008 & & & & \begin{tabular}{|l|}
0.018 \\
\end{tabular} & & 0.030 \\
\hline Batch 2272 & 0.007 & & & & \begin{tabular}{|l|}
0.018 \\
\end{tabular} & & 0.030 \\
\hline Batch 3171 & 0.011 & & & & 0.015 & & 0.030 \\
\hline Batch 3172 & 0.012 & & & & \begin{tabular}{|l|}
0.015 \\
\end{tabular} & & 0.030 \\
\hline Batch 3271 & 0.018 & & & & 0.015 & & 0.030 \\
\hline Batch 3272 & 0.021 & & & & 0.015 & & 0.040 \\
\hline Batch 4171 & 0.010 & & & & \begin{tabular}{|l|}
0.026 \\
\end{tabular} & & 0.040 \\
\hline Batch 4172 & 0.007 & & & & 0.026 & & 0.030 \\
\hline Batch 4272 & 0.008 & & & & \begin{tabular}{|l|}
0.025 \\
\end{tabular} & & 0.030 \\
\hline HM 171 & 0.016 & & & & \begin{tabular}{|l|}
0.007 \\
\end{tabular} & & 0.020 \\
\hline HM 172 & 0.017 & & & & \begin{tabular}{|l|}
0.006 \\
\end{tabular} & & 0.020 \\
\hline HM 271 & 0.026 & & & & \begin{tabular}{|l|}
0.005 \\
\end{tabular} & & 0.030 \\
\hline HM 272 & 0.027 & & & & \begin{tabular}{|l|}
0.005 \\
\end{tabular} & & 0.030 \\
\hline
\end{tabular}


Table 3.3. Residual Glass Composition (in mass fractions of oxides): Glasses Precipitating Clinopyroxene

\begin{tabular}{|c|c|c|c|c|c|c|c|c|c|c|c|}
\hline Glass & $\mathrm{Al}_{2} \mathrm{O}_{3}$ & $\mathbf{B}_{2} \mathbf{O}_{3}$ & $\mathrm{CaO}$ & $\mathrm{Fe}_{2} \mathrm{O}_{3}$ & $\mathbf{L i}_{2} \mathbf{O}$ & MgO & $\mathrm{Na}_{2} \mathrm{O}$ & $\mathrm{SiO}_{2}$ & $\mathrm{ZrO}_{2}$ & Other & Sum \\
\hline CVS1-1 & 0.068 & 0.123 & 030 & 0.035 & 0.040 & 039 & 0.098 & 0.477 & 0.046 & 0.044 & 100 \\
\hline CVS1-8 & 000 & 061 & 000 & 0.166 & 0.012 & 083 & 096 & 0.553 & 0.017 & 012 & 000 \\
\hline CVS1-15 & .000 & 051 & .000 & 0.021 & 0.063 & .082 & 0.124 & 0.556 & 0.000 & .102 & .000 \\
\hline CVS1-19 & 0.070 & 125 & 0.030 & 0.028 & 0.041 & 0.040 & 0.097 & 0.476 & 0.047 & 045 & .000 \\
\hline-20 & 070 & 125 & 030 & 0.028 & 0.041 & 040 & 097 & 0.476 & 0.047 & 045 & .00 \\
\hline-22 & .000 & 060 & 000 & 0.168 & 0.012 & .083 & 0.096 & 0.553 & 0.016 & 012 & .000 \\
\hline CVS1-23 & 0.047 & 0.098 & 0.030 & 0.112 & 0.038 & 0.009 & 0.104 & 0.515 & 0.006 & 0.040 & .000 \\
\hline CVS2-2 & 116 & 074 & 074 & 0.029 & 0.063 & .000 & 0.086 & 0.501 & 0.032 & 026 & .000 \\
\hline CVS2-25 & 004 & 064 & 103 & 0.106 & 0.047 & .000 & 0.094 & 0.510 & 0.000 & .073 & .000 \\
\hline CVS2-36 & 0.009 & 0.054 & 0.086 & 0.143 & 0.068 & 0.000 & 0.089 & 0.541 & 0.000 & 0.011 & .000 \\
\hline 42 & 044 & 074 & 047 & 0.046 & 0.042 & 056 & 53 & 0.563 & 0.030 & 444 & 80 \\
\hline 50 & 72 & 130 & 31 & 7 & 0 & 41 & 96 & 75 & 49 & 46 & 00 \\
\hline CVS2-71 & 0.029 & 0.051 & 0.003 & 0.081 & 0.064 & 0.001 & 0.093 & 0.570 & 0.043 & 0.066 & 1.000 \\
\hline CVS2-96 & 075 & 134 & 032 & 0.006 & 0.044 & 043 & 0.094 & 0.473 & 0.050 & 048 & .000 \\
\hline Ble & 43 & & 11 & 98 & 0 . & 14 & 92 & 0.528 & 001 & & 00 \\
\hline Blend 1172 & 0.043 & 0.082 & 0.011 & 0.099 & 0.045 & 0.014 & 0.092 & 0.528 & 0.001 & 0.084 & 1.000 \\
\hline Blend 1272 & 043 & 083 & 011 & 0.096 & 0.046 & 014 & 0.093 & 0.530 & 0.001 & 084 & .000 \\
\hline Batch 1171 & 0.050 & 0.080 & 0.013 & 0.117 & 0.045 & 0.015 & 0.091 & 0.513 & 0.001 & 0.076 & 1.000 \\
\hline Batch 1271 & 0.050 & 0.080 & 0.013 & 0.118 & 0.045 & 0.015 & 0.091 & 0.513 & 0.001 & 0.075 & 1.000 \\
\hline Batch 1272 & 50 & 080 & 13 & 0.118 & 0.045 & 15 & 0.091 & 0.513 & 01 & 0.075 & 000 \\
\hline Batch 2171 & 0.048 & 0.081 & 0.011 & 0.099 & 0.046 & 0.015 & 0.093 & 0.530 & 0.002 & 0.076 & 1.000 \\
\hline Batch 2172 & 0.048 & 0.081 & 0.011 & 0.097 & 0.046 & 0.015 & 0.093 & 0.531 & 0.002 & 0.075 & 1.000 \\
\hline Batch 2271 & 0.048 & 0.081 & 0.011 & 0.099 & 0.046 & 0.015 & 0.093 & 0.531 & 0.002 & 0.075 & 1.000 \\
\hline Batch 2272 & 0.047 & 0.081 & 0.011 & 0.099 & 0.046 & 0.015 & 0.093 & 0.531 & 0.002 & 0.075 & 1.000 \\
\hline Batch 3171 & 0.035 & 0.079 & 0.010 & 0.106 & 0.046 & 0.015 & 0.091 & 0.538 & 0.001 & 0.078 & 1.000 \\
\hline Batch 3172 & 0.035 & 0.079 & 0.010 & 0.106 & 0.046 & 0.015 & 0.091 & 0.538 & 0.001 & 0.078 & 1.000 \\
\hline Batch 3271 & 0.036 & 0.080 & 0.010 & 0.104 & 0.047 & 0.015 & 0.091 & 0.539 & 0.001 & 0.079 & 1.000 \\
\hline Batch 3272 & 0.036 & 0.080 & 0.010 & 0.103 & 0.047 & 0.015 & 0.091 & 0.539 & 0.001 & 0.079 & 1.000 \\
\hline Batch 4171 & 0.036 & 0.084 & 0.009 & 0.100 & 0.045 & 0.015 & 0.094 & 0.515 & 0.002 & 0.102 & 1.000 \\
\hline Batch 4172 & 0.035 & 0.084 & 0.009 & 0.100 & 0.044 & 0.015 & 0.094 & 0.515 & 0.002 & 0.101 & 1.000 \\
\hline Batch 4272 & 0.035 & 0.084 & 0.009 & 0.101 & 0.044 & 0.015 & 0.094 & 0.514 & 0.002 & 0.102 & 1.000 \\
\hline HM 171 & 0.073 & 0.072 & 0.010 & 0.069 & 0.047 & 0.015 & 0.085 & 0.558 & 0.003 & 0.066 & 1.000 \\
\hline HM 172 & 0.073 & 0.072 & 0.010 & 0.069 & 0.047 & 0.015 & 0.085 & 0.558 & 0.003 & 0.066 & 1.000 \\
\hline HM 271 & 0.074 & 0.073 & 0.010 & 0.067 & 0.048 & 0.015 & 0.085 & 0.558 & 0.003 & 0.067 & 1.000 \\
\hline HM 272 & 0.074 & 0.073 & 0.010 & 0.067 & 0.048 & 0.015 & 0.085 & 0.558 & 0.003 & 0.067 & 1.000 \\
\hline
\end{tabular}


Table 3.4. Measured $r_{B}$ and $r_{N a}$ (in $g / \mathrm{m}^{2}$ ) from Quenched and Heat-Treated Glasses with Clinopyroxene

\begin{tabular}{|l|c|c|c|c||}
\hline Glass & $\mathbf{Q}, \mathbf{M}$ & $\mathbf{Q}, \mathbf{M}$ & $\mathbf{H}, \mathbf{M}$ & $\mathbf{H}, \mathbf{M}$ \\
\hline & $\boldsymbol{r}_{\boldsymbol{B}}$ & $\boldsymbol{r}_{\boldsymbol{N a}}$ & $\boldsymbol{r}_{\boldsymbol{h} \boldsymbol{B}}$ & $\boldsymbol{r}_{\boldsymbol{h} \boldsymbol{\boldsymbol { a }}}$ \\
\hline CVS1-1 & 0.52 & 0.40 & 0.37 & 0.36 \\
\hline CVS1-8 & 1.24 & 0.81 & 4.18 & 1.66 \\
\hline CVS1-15 & 18.78 & 14.00 & 19.61 & 14.02 \\
\hline CVS1-19 & 0.52 & 0.43 & 0.41 & 0.36 \\
\hline CVS1-20 & 0.46 & 0.40 & 0.44 & 0.36 \\
\hline CVS1-22 & 1.12 & 0.76 & 3.86 & 1.54 \\
\hline CVS1-23 & 0.53 & 0.49 & 0.37 & 0.34 \\
\hline CVS2-2 & 0.13 & 0.19 & 0.14 & 0.16 \\
\hline CVS2-25 & 4.66 & 3.53 & 1.47 & 1.03 \\
\hline CVS2-36 & 0.48 & 0.65 & 0.35 & 0.43 \\
\hline CVS2-42 & 1.69 & 1.34 & 6.11 & 3.76 \\
\hline CVS2-50 & 0.44 & 0.37 & 0.56 & 0.38 \\
\hline CVS2-71 & 0.41 & 0.35 & 0.37 & 0.32 \\
\hline CVS2-96 & 0.49 & 0.41 & 0.66 & 0.35 \\
\hline Blend 1171 & 0.30 & 0.31 & 0.36 & 0.33 \\
\hline Blend 1172 & 0.31 & 0.32 & 0.36 & 0.32 \\
\hline Blend 1272 & - & - & 0.39 & 0.33 \\
\hline Batch 1171 & 0.32 & 0.32 & 0.34 & 0.34 \\
\hline Batch 1271 & 0.34 & 0.33 & 0.41 & 0.37 \\
\hline Batch 1272 & - & - & 0.33 & 0.32 \\
\hline Batch 2171 & 0.28 & 0.30 & 0.31 & 0.28 \\
\hline Batch 2172 & 0.27 & 0.28 & 0.30 & 0.29 \\
\hline Batch 2271 & 0.27 & 0.28 & 0.35 & 0.30 \\
\hline Batch 2272 & - & - & 0.35 & 0.30 \\
\hline Batch 3171 & 0.38 & 0.37 & 0.43 & 0.39 \\
\hline Batch 3172 & 0.37 & 0.36 & 0.44 & 0.39 \\
\hline Batch 3271 & 0.36 & 0.35 & 0.39 & 0.35 \\
\hline Batch 3272 & - & - & 0.40 & 0.36 \\
\hline Batch 4171 & 0.41 & 0.40 & 0.42 & 0.39 \\
\hline Batch 4172 & 0.41 & 0.40 & 0.46 & 0.44 \\
\hline Batch 4272 & - & - & 0.47 & 0.44 \\
\hline HM 171 & 0.27 & 0.22 & 0.29 & 0.24 \\
\hline HM 172 & 0.26 & 0.21 & 0.26 & 0.21 \\
\hline HM 271 & 0.27 & 0.22 & 0.29 & 0.24 \\
\hline HM 272 & - & - & 0.29 & 0.23 \\
\hline \hline
\end{tabular}


Table 3.5. Calculated $r_{B}$ and $r_{N a}\left(\right.$ in $\mathrm{g} / \mathrm{m}^{2}$ ) from Quenched and HeatTreated Glasses with Clinopyroxene

\begin{tabular}{|c|c|c|c|c|c|c|}
\hline Glass & $\mathbf{Q}, \mathbf{C}$ & $\mathbf{Q}, \mathbf{C}$ & H, QC, RC & $\mathrm{H}, \mathrm{QC}, \mathrm{RC}$ & H, QM, RC & H, QM, RC \\
\hline & $r_{B}$ & $r_{N a}$ & $r_{r B}$ & $\boldsymbol{r}_{r N a}$ & $\boldsymbol{r}_{r B}$ & $\boldsymbol{r}_{r N a}$ \\
\hline & & & \multicolumn{2}{|c|}{ with $r_{j}$ calculated } & \multicolumn{2}{|c|}{ with $r_{j}$ measured } \\
\hline CVS1-1 & 0.84 & 0.69 & 1.15 & 0.95 & 1.59 & 1.31 \\
\hline CVS1-8 & 0.63 & 0.51 & 1.43 & 1.17 & 3.27 & 2.67 \\
\hline CVS1-15 & 15.23 & 12.05 & 15.05 & 11.91 & 14.88 & 11.77 \\
\hline CVS1-19 & 0.84 & 0.69 & 1.27 & 1.05 & 1.94 & 1.59 \\
\hline CVS1-20 & 0.84 & 0.69 & 1.27 & 1.05 & 1.94 & 1.59 \\
\hline CVS1-22 & 0.63 & 0.51 & 1.29 & 1.05 & 2.64 & 2.16 \\
\hline CVS1-23 & 0.87 & 0.73 & 0.97 & 0.81 & 1.08 & 0.91 \\
\hline CVS2-2 & 0.10 & 0.11 & 0.13 & 0.14 & 0.16 & 0.18 \\
\hline CVS2-25 & 1.25 & 1.37 & 2.15 & 2.35 & 3.69 & 4.04 \\
\hline CVS2-36 & 1.01 & 1.15 & 1.26 & 1.44 & 1.56 & 1.79 \\
\hline CVS2-42 & 0.72 & 0.60 & 2.34 & 1.92 & 7.54 & 6.20 \\
\hline CVS2-50 & 0.84 & 0.69 & 1.49 & 1.23 & 2.66 & 2.19 \\
\hline CVS2-71 & 0.80 & 0.54 & 0.80 & 0.54 & 0.80 & 0.54 \\
\hline CVS2-96 & 0.84 & 0.69 & 1.77 & 1.46 & 3.74 & 3.08 \\
\hline Blend 1171 & 0.99 & 0.71 & 1.09 & 0.78 & 1.20 & 0.86 \\
\hline Blend 1172 & 0.99 & 0.71 & 1.07 & 0.77 & 1.17 & 0.84 \\
\hline Blend 1272 & 0.99 & 0.71 & 1.10 & 0.78 & 1.22 & 0.87 \\
\hline Batch 1171 & 0.77 & 0.56 & 0.85 & 0.61 & 0.94 & 0.68 \\
\hline Batch 1271 & 0.77 & 0.56 & 0.85 & 0.61 & 0.93 & 0.67 \\
\hline Batch 1272 & 0.77 & 0.56 & 0.85 & 0.61 & 0.93 & 0.67 \\
\hline Batch 2171 & 0.87 & 0.63 & 0.96 & 0.69 & 1.06 & 0.77 \\
\hline Batch 2172 & 0.87 & 0.63 & 0.97 & 0.70 & 1.10 & 0.79 \\
\hline Batch 2271 & 0.87 & 0.63 & 0.96 & 0.69 & 1.06 & 0.77 \\
\hline Batch 2272 & 0.87 & 0.63 & 0.95 & 0.69 & 1.05 & 0.76 \\
\hline Batch 3171 & 1.06 & 0.76 & 1.17 & 0.84 & 1.30 & 0.93 \\
\hline Batch 3172 & 1.06 & 0.76 & 1.18 & 0.84 & 1.31 & 0.94 \\
\hline Batch 3271 & 1.06 & 0.76 & 1.21 & 0.87 & 1.39 & 0.99 \\
\hline Batch 3272 & 1.06 & 0.76 & 1.23 & 0.88 & 1.42 & 1.02 \\
\hline Batch 4171 & 1.25 & 0.87 & 1.44 & 1.00 & 1.65 & 1.14 \\
\hline Batch 4172 & 1.25 & 0.87 & 1.42 & 0.98 & 1.61 & 1.11 \\
\hline Batch 4272 & 1.25 & 0.87 & 1.42 & 0.98 & 1.61 & 1.12 \\
\hline HM 171 & 0.36 & 0.27 & 0.40 & 0.30 & 0.44 & 0.33 \\
\hline HM 172 & 0.36 & 0.27 & 0.40 & 0.30 & 0.44 & 0.33 \\
\hline HM 271 & 0.36 & 0.27 & 0.41 & 0.31 & 0.47 & 0.35 \\
\hline
\end{tabular}




\begin{tabular}{|l|l|l|l|l|l|l|}
\hline HM 272 & 0.36 & 0.27 & 0.42 & 0.31 & 0.48 & 0.36 \\
\hline
\end{tabular}

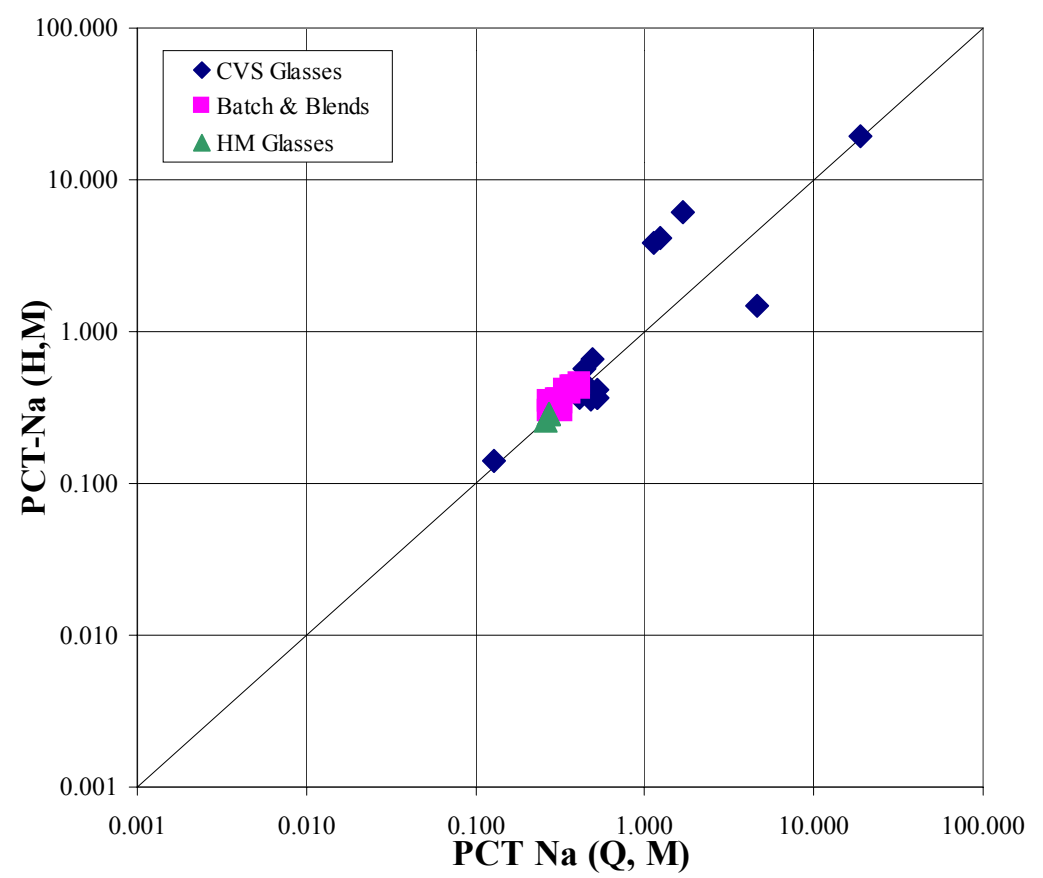

Figure 3-1. Glasses Precipitating Clinopyroxene: $r_{h B}$ vs. $r_{B}$

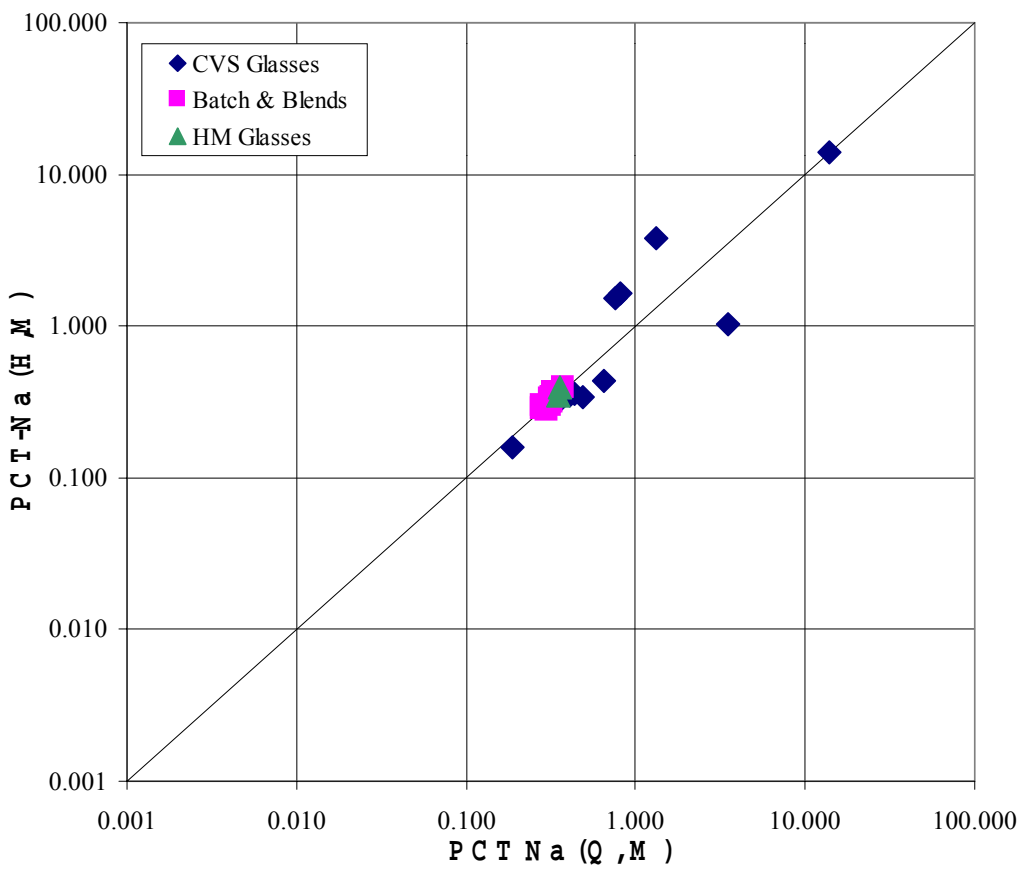

Figure 3-2. Glasses Precipitating Clinopyroxene: $r_{\mathrm{hNa}}$ vs. $\boldsymbol{r}_{\mathrm{Na}}$ 


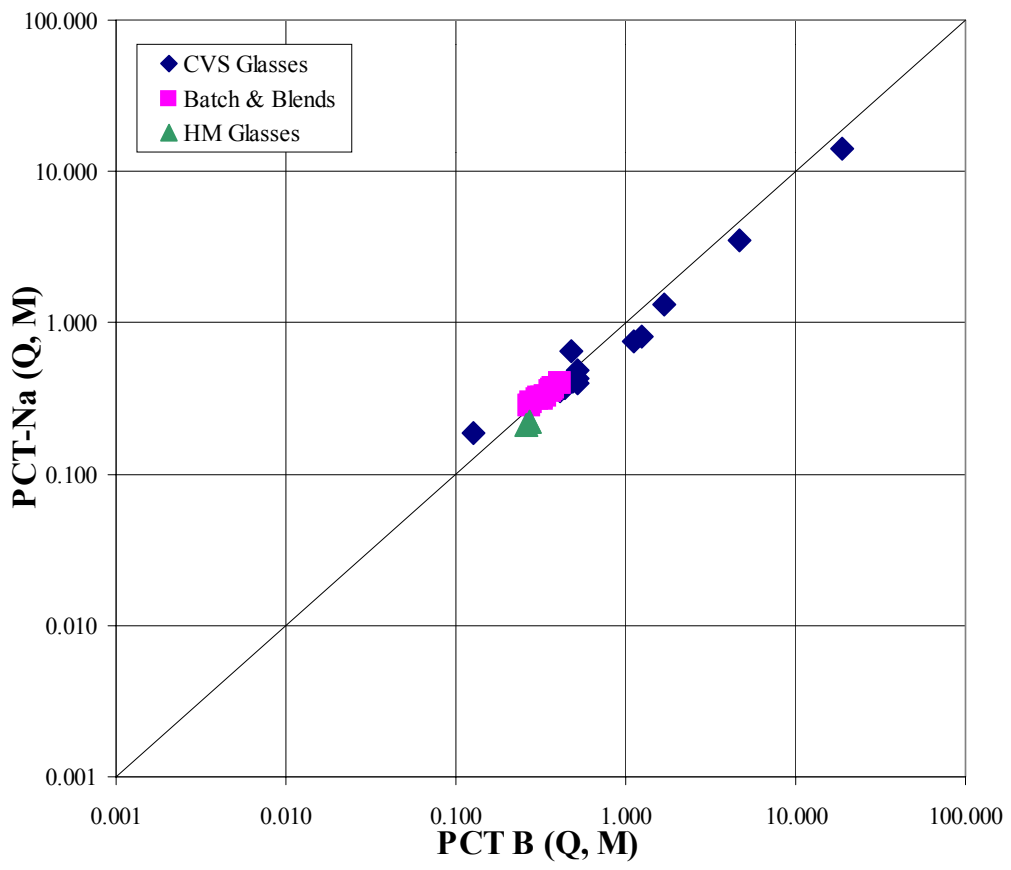

Figure 3-3. Glasses Precipitating Clinopyroxene: $r_{N a}$ vs. $r_{B}$

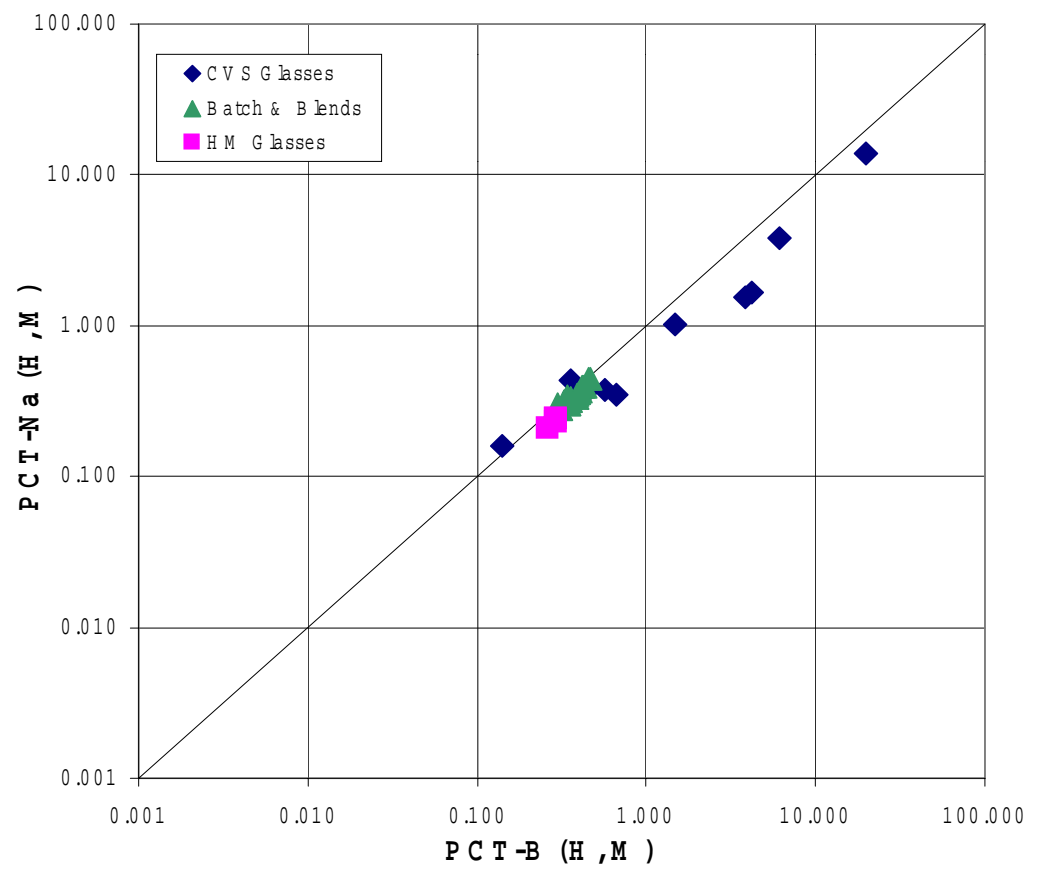

Figure 3-4. Glasses Precipitating Clinopyroxene: $r_{h N a}$ vs. $r_{h B}$ 


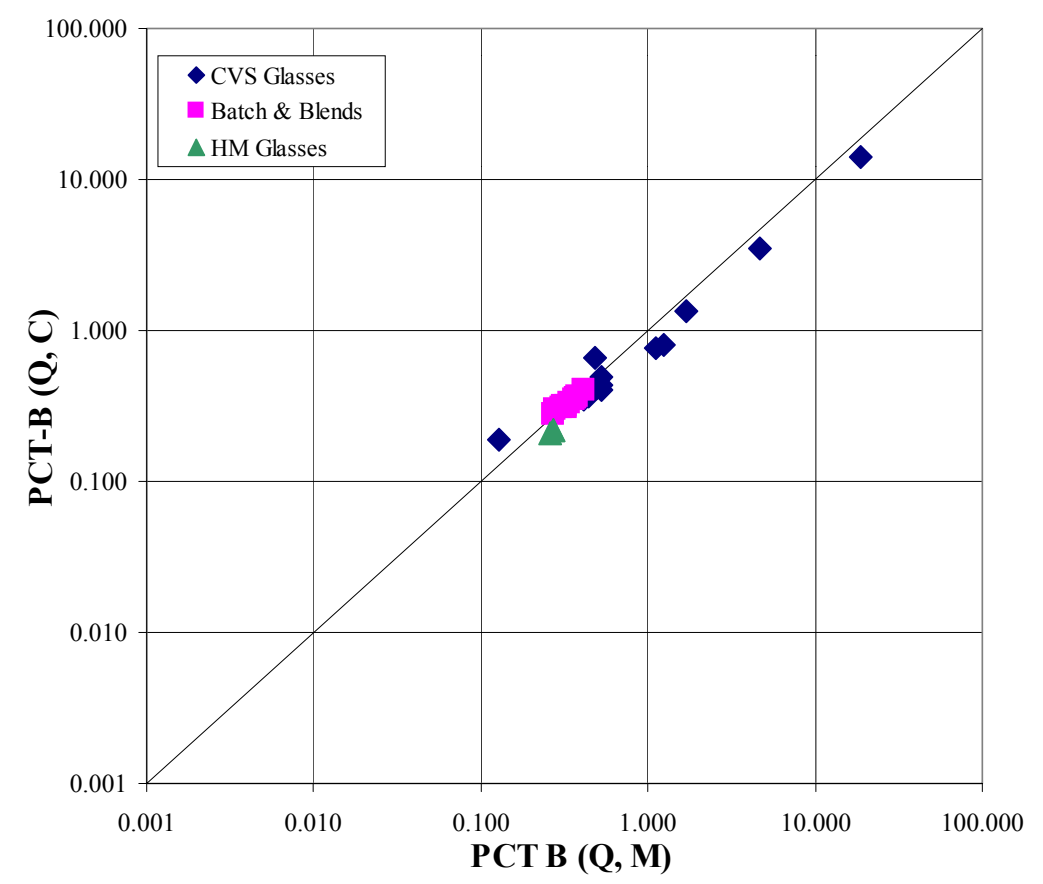

Figure 3-5. Glasses Precipitating Clinopyroxene: $r_{B^{\prime}}$ Calculated vs. Measured

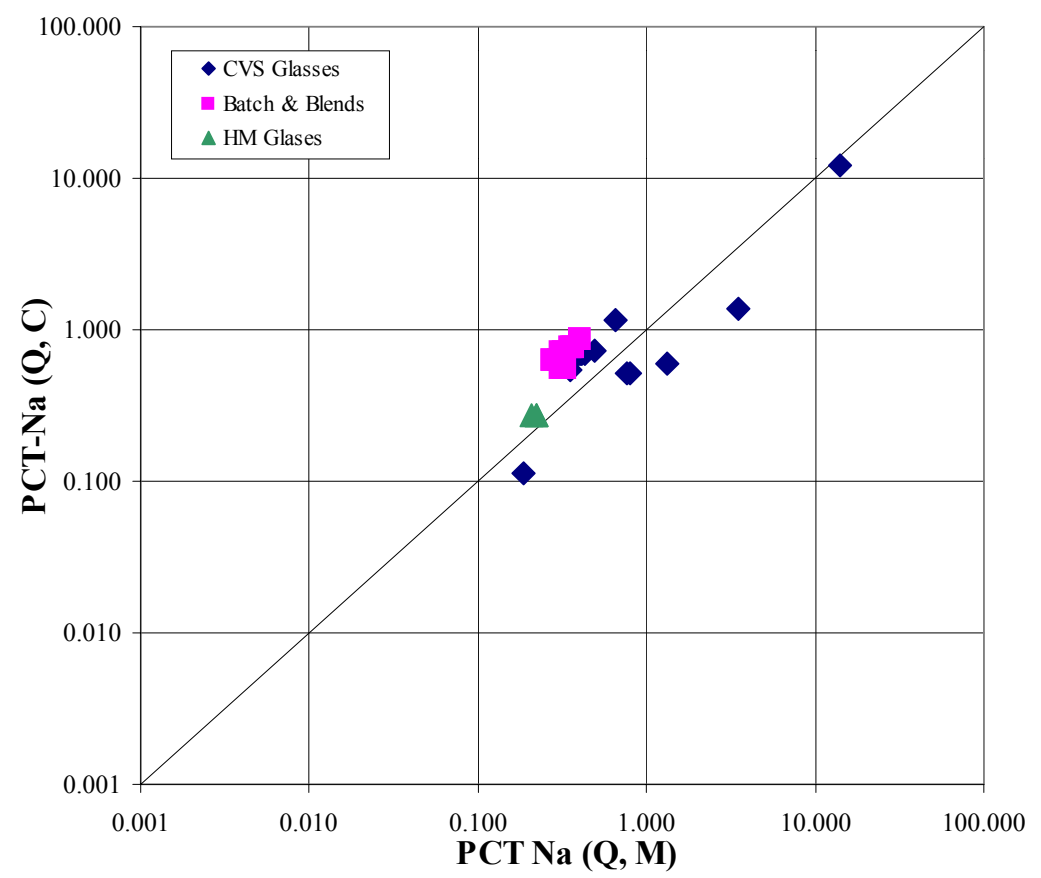

Figure 3-6. Glasses Precipitating Clinopyroxene: $r_{\mathrm{Na}}$, Calculated vs. 
Measured

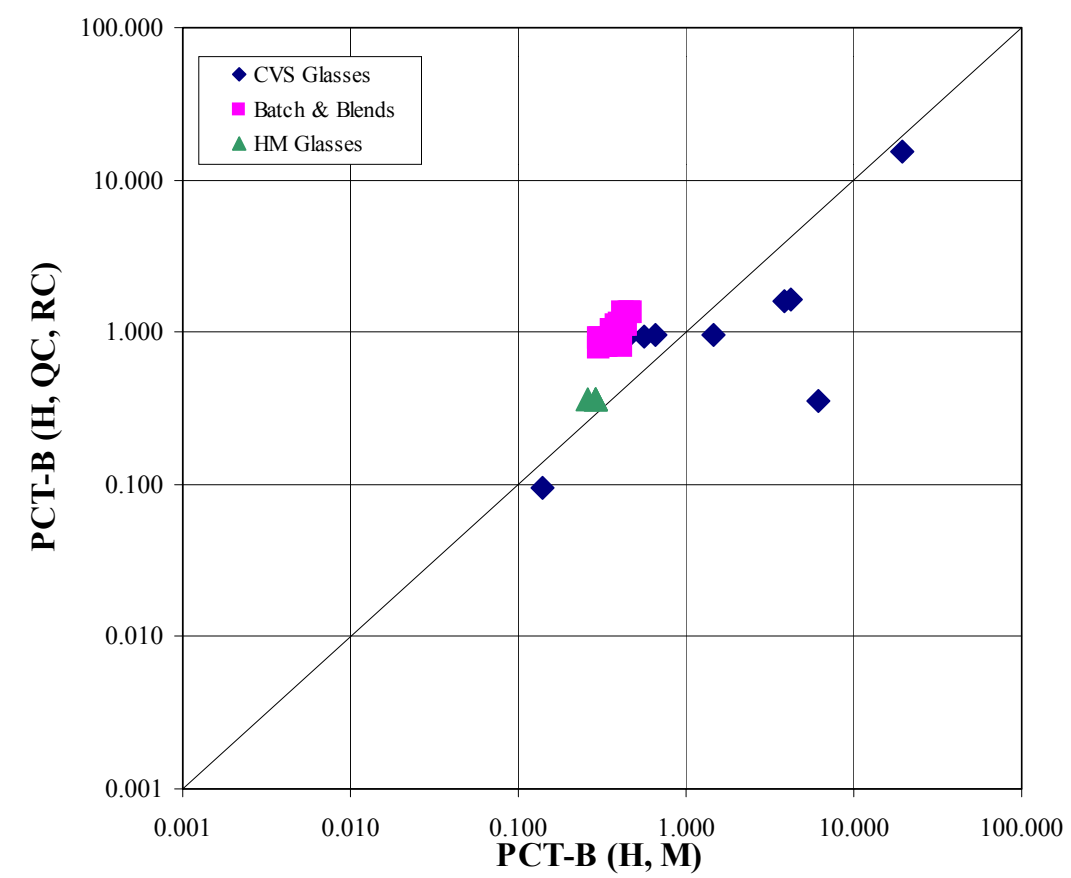

Figure 3-7. Glasses Precipitating Clinopyroxene: $r_{r B}$ (calculated) vs . $r_{h B}$ (measured)

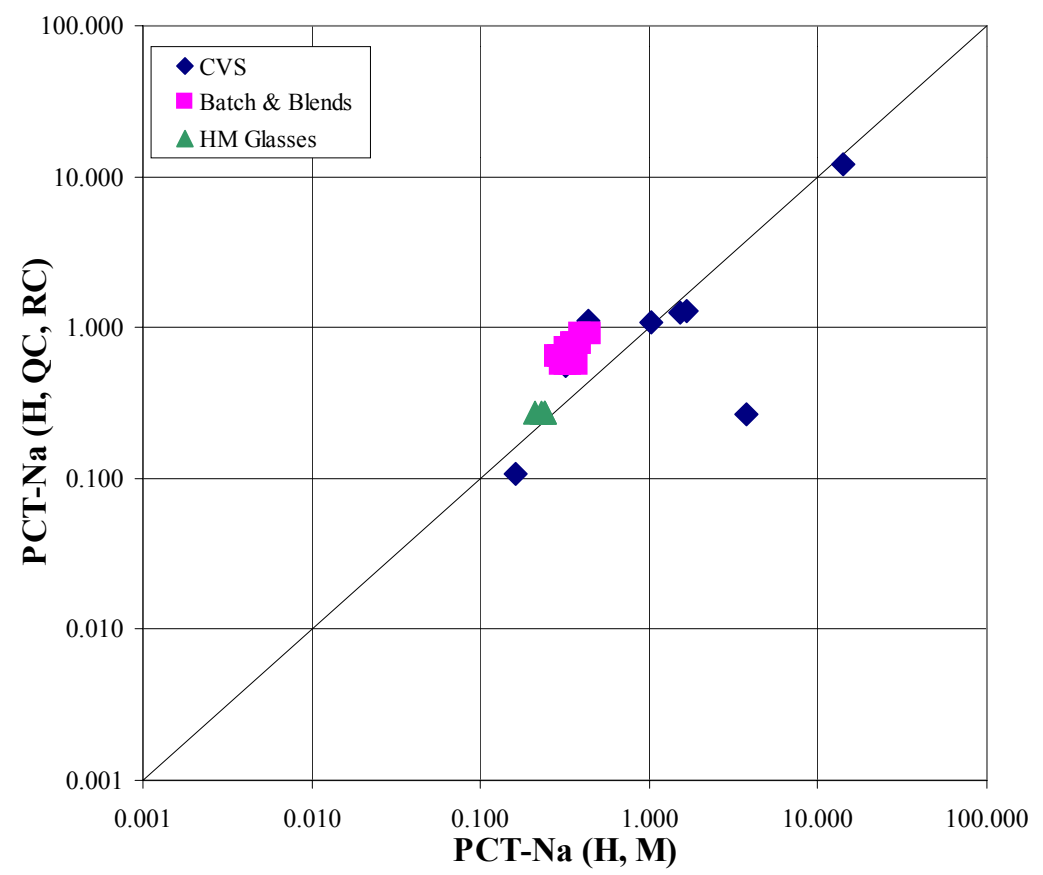


Figure 3-8. Glasses Precipitating Clinopyroxene: $r_{r N a}$ (calculated) vs. $r_{h N a}$ (measured)

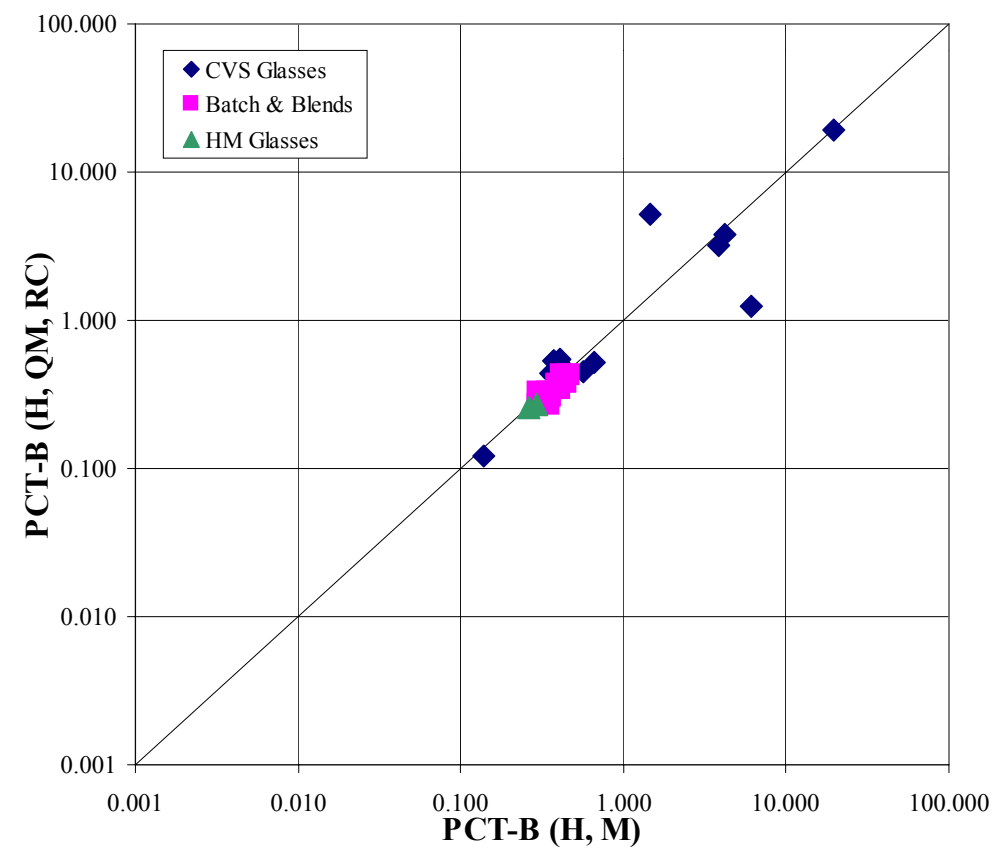

Figure 3-9. Glasses Precipitating Clinopyroxene: $r_{x B}$ (calculated using measured $r_{B}$ ) vs. $r_{h B}$ (measured)

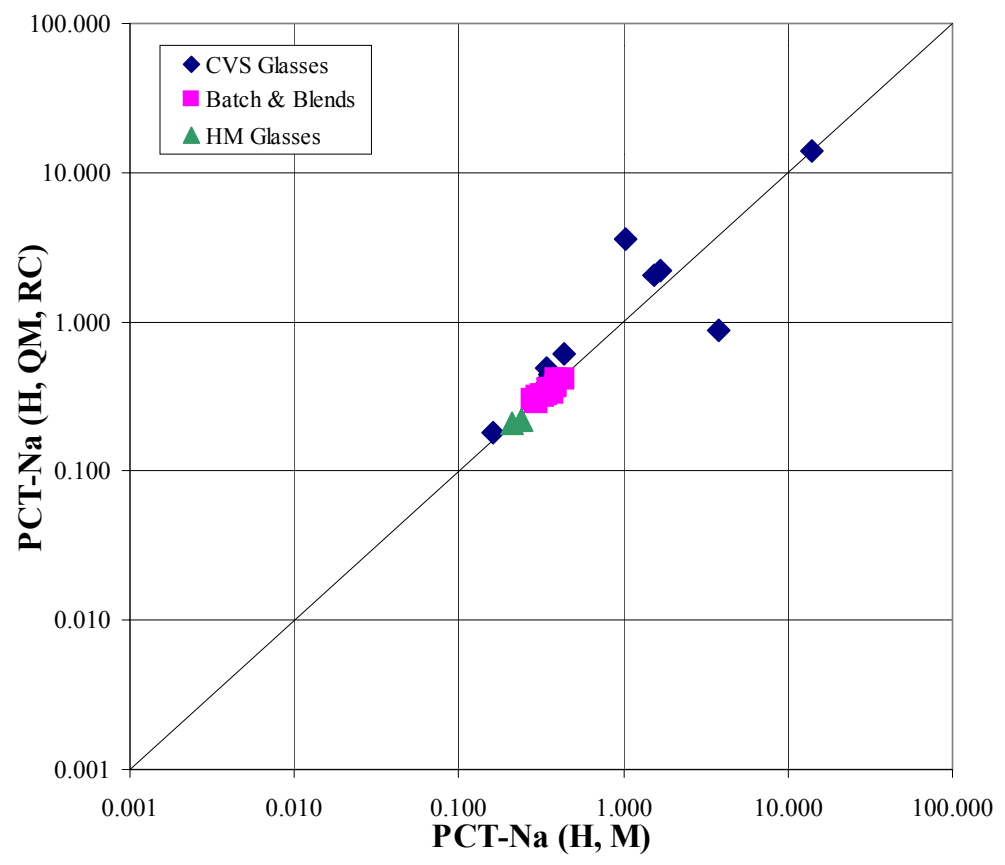


Figure 3-10. Glasses Precipitating Clinopyroxene: $r_{\mathrm{rNa}}$ (calculated using measured $r_{N a}$ )

vs. $\boldsymbol{r}_{h N a}$ (measured) 


\subsection{Glasses Precipitating NP}

As explained in Section 3, NP has potentially the most detrimental impact on dissolution of Hanford and Savannah River HLW glasses. Similar to natural NP, NP from selected HLW glass samples was found to contain excess $\mathrm{SiO}_{2}$ and $\mathrm{Fe}_{2} \mathrm{O}_{3}$ (Menkhaus et al. 2000). However, the impact of this NP on PCT is expected to be similar to that of stoichiometric $\mathrm{NP}\left(\mathrm{NaAlSiO}_{4}\right)$ - see Table 2.3. Table 3.6 lists compositions of glasses that precipitated NP upon heat treatment, CCC, or IHT. Nine of these glasses are from the CVS (Hrma et al. 1994; Vienna et al. 1996) and 15 are from the NP study (Li et al. 1997).

Not all the glasses listed in Table 3.6 fall into the composition region defined in Table 2.2. Compositions CVS3-16, NP-B-1, NP-Li-1, NP-Al-1, and NP-Al-2 are outside of the composition region on which the component coefficients listed in Table 2.1 are valid. The most deviant composition, CVS316 , is a high-temperature glass (melting above $1200^{\circ} \mathrm{C}$ ) with low B and high Al concentrations. Further, NP-B-1 is a B-free glass, NP-Li-1 is a Li-free glass, and glasses NP-Al-1, NP-Al-2, NP-B-1, and NP-Li-1 have elevated $\mathrm{Na}$ concentrations ( 21 to 22 mass $\% \mathrm{Na}_{2} \mathrm{O}$ ). Also, note that several NP glasses have low $\mathrm{Si}$ concentrations ( 33 to 39 mass $\% \mathrm{SiO}_{2}$ ). The component coefficients listed in Table 2.1 were used to predict $r_{B}$ and $r_{N a}$ for these glasses.

Table 3.7 summarizes mass fractions of crystalline phases after CCC heat treatment. Several glasses precipitated more than one crystalline phase. Five compositions that precipitated NP under IHT, but did not precipitate any NP under CCC, are also included (CVS2-34, CVS2-98, NP-Al-1, NP-Si-3, and NP-Si4) in the table. Table 3.8 and Table 3.9 list residual glass compositions computed with Equation (3). Table 3.8 is based on stoichiometric NP (NP $1, \mathrm{NaAlSiO}_{4}$ ) and Table 3.9 is based on a measured NP composition (NP 2, see Table 2.3). Several compositions that were originally close to the qualified composition region boundary moved outward or inward (within the composition space) during heat treatment (as a result of crystallization), according to whether the particular component was close to the upper or lower limit and whether this component did or did not participate in the formation of a crystalline phase. The calculated content of $\mathrm{Na}_{2} \mathrm{O}$ and $\mathrm{Fe}_{2} \mathrm{O}_{3}$ was also affected by the choice of $\mathrm{NP}$ composition. In particular, excess $\mathrm{Fe}_{2} \mathrm{O}_{3}$ appeared in residual glass with stoichiometric NP. Several residual glasses had excess $\mathrm{Na}_{2} \mathrm{O}$. This excess was greater when measured NP composition (NP 2) was used for calculations.

The extreme glass, CVS3-16, precipitated roughly 73 mass $\%$ crystals (eucryptite and NP). The residual "glass" contained only 3.5 mass $\%$ or 8.5 mass $\% \mathrm{SiO}_{2}$, according to which NP was used, and a negligible content of $\mathrm{B}_{2} \mathrm{O}_{3}$. Thus, the residual phase falls well outside the composition region of typical glass-forming materials. Therefore, the CVS3-16 composition is dropped from further discussion (the estimated $r_{r B}$ and $r_{r N a}$ values for this composition, though shown, are meaningless). Several residual glasses (CVS2-63, CVS2-85, NP-Al-3, NP-Al-4, NP-K-2, and NP-Li-2) have low silica concentrations, 19 to 35 mass \% based on NP 1, or 14 to 29 mass\% based on NP 2. These residual glass compositions are 7 to 28 mass\% below the lower $\mathrm{SiO}_{2}$ limit for the model validity. 
Measured and calculated $r_{B}$ and $r_{N a}$ from quenched and heat-treated glasses are summarized in Table 3.10 and Table 3.11. Releases from heat-treated glasses were calculated using Equation (5) with either the measured or calculated release values from quenched glasses. Data listed in Table 3.10 and Table 3.11 are compared in Figure 3-11 through Figure 3-20. As Figure 3-11 and Figure 3-12 show, heat treatment (CCC or IHT) strongly affects $r_{B}$ and $r_{N a}$ from most of the NP-precipitating glasses. Glasses that were not affected by heat treatment are

- two CVS compositions that did not precipitate any NP or other crystalline phases on CCC (CVS2-34 and CVS2-98; these glasses were automatically included because they precipitated NP when heated treated isothermally)

- NP glasses that did not precipitate any crystalline phases on IHT (NP-Al-1, NP-Si-3, and NP-Si-4; these glasses were included because they were within or close to the NP region on the $\mathrm{Na}_{2} \mathrm{O}-\mathrm{Al}_{2} \mathrm{O}_{3}$ $\mathrm{SiO}_{2}$ phase diagram—see Li et al. 1997)

- one NP glass that precipitated both NP and lithium silicates (NP-Li-2). Heat treatment according to the CCC schedule had a very small impact on $r_{B}$ and $r_{N a}$ from CVS2-86 glass that formed only 5 mass $\%$ NP.

Interestingly, CVS2-86 and CVS2-85 glasses had similar compositions except that CVS2-86 had a higher $\mathrm{CaO}$ concentration, a lower $\mathrm{MgO}$ concentration, and nearly double the $\mathrm{Li}_{2} \mathrm{O}$ concentration (Table 3.6). However, CVS2-85 glass precipitated 54 mass $\%$ NP and 21\% lithium silicate, whereas CVS2-86 glass precipitated merely 5 mass $\%$ NP.

Another anomalous glass is NP-Li-2. It is tempting to attribute the little impact of heat treatment on $r_{B}$ and $r_{N a}$ from this glass to the precipitation of lithium silicate that could offset the effect of NP. However, calculations do not support this idea (compare the predicted releases listed in Table 3.11 with the measured values listed in Table 3.10). The calculated results are understandable considering the composition of the residual NP-Li-2 glass: the residual glass contains only 14 mass $\% \mathrm{SiO}_{2}$ and has 33 mass $\% \mathrm{Na}_{2} \mathrm{O}$ (Table 3.9). The measured $r_{h B}$ and $r_{h N a}$ values indicate that this composition of residual phase is unlikely; this could be caused by exaggerated values of crystallinity fractions.

Overall, $r_{B}$ from quenched glasses are not higher than $5 \mathrm{~g} / \mathrm{m}^{2}$ and $r_{N a}$ are not higher than $3 \mathrm{~g} / \mathrm{m}^{2}$. The release values from heat-treated glasses with more than 10 mass $\% \mathrm{NP}$ are as high as $48 \mathrm{~g} / \mathrm{m}^{2}$ for $r_{h B}$ and $28 \mathrm{~g} / \mathrm{m}^{2}$ for $r_{h N a}$ (Table 3.10).

The glasses with the largest differences between releases from quenched $\left(r_{B}\right.$ and $\left.r_{N a}\right)$ and heat-treated $\left(r_{h B}\right.$ and $\left.r_{h N a}\right)$ samples are NP-K-1, NP-K-2, NP-A1-3, NP-A1-4, CVS2-85, NP-Na-2, CVS2-35, and CVS1-16. These glasses precipitated 15 to 40 mass $\%$ NP. The $r_{B}$ values from these glasses reached the level of $22 \mathrm{~g} / \mathrm{m}^{2}$ to $48 \mathrm{~g} / \mathrm{m}^{2}$. Their $r_{N a}$ were lower $\left(9 \mathrm{~g} / \mathrm{m}^{2}\right.$ to $\left.28 \mathrm{~g} / \mathrm{m}^{2}\right)$ because Na was partitioned between residual glass and NP, a relatively more durable phase (the measured $r_{h B}$ and $r_{h N a}$ were taken as reported; they were not renormalized with respect to the calculated composition of residual glass). 
Figure 3-13 compares $r_{N a}$ with $r_{B}$ from quenched glasses (excluding CVS3-16). Sodium releases $\left(r_{N a}\right)$ are close to $r_{B}$ and tend to be somewhat higher for glasses with $\left.r_{B}<1 \mathrm{~g} / \mathrm{m}^{2}\right)$. This is a consequence of enhanced $\mathrm{Na}$ leaching at the early stages of dissolution. Figure 3-14 compares $r_{N a}$ and $r_{B}$ from heat-treated glasses. With exception of CVS3-16 glass, the values are close, but values of $r_{h N a}$ show a tendency to lower values as compared to $r_{h B}$, especially in glasses with $r_{h B}>10 \mathrm{~g} / \mathrm{m}^{2}$. This is as expected because part of the Na was bonded in NP ( $r_{h B}$ and $r_{h N a}$ were not renormalized with respect to the calculated composition of residual glass).

As Figure 3-15 and Figure 3-16 show, $r_{B}$ and $r_{N a}$ are overpredicted by an order of magnitude for three glasses and underpredicted (by two orders of magnitude) for one glass. The three glasses with overpredicted $r_{B}$ and $r_{\mathrm{Na}}$ are CVS2-33, NP-Al-1, and NP-Al-2. These glasses (Table 3.6) are high in alkalis, nearly 27 mass $\% \mathrm{Na}_{2} \mathrm{O}+\mathrm{Li}_{2} \mathrm{O}$ in the two NP glasses. It is generally assumed that glasses with more than 25 mass \% alkali oxides are non-durable. However, the lower-than-predicted measured $r_{B}$ and $r_{N a}$ from these three glasses suggest that it is possible to formulate durable high-alkali glasses by adding $\mathrm{Al}_{2} \mathrm{O}_{3}$. This effect is nonlinear because it is not predicted when first-order models based on medium-alkali glasses are to be extrapolated to the high-Na, high-Li region. The low $r_{B}$ and $r_{N a}$ from quenched NP-Al-1 glass did not increase on heat treatment because NP-Al-1 is one of the glasses that did not precipitate any crystalline phase upon heat treatment (all NP-denoted glasses were automatically included in this section).

When measured $r_{B}$ and $r_{N a}$ from quenched glasses are used in Equation (5), the calculated $r_{r B}$ and $r_{r N a}$ from heat-treated glasses (based on residual glass compositions) are in better agreement with measured $r_{h B}$ and $r_{h N a}$ values (Figure 3-19 and Figure 3-20). This indicates that the difference between calculated and measured $r_{B}$ and $r_{N a}$ from quenched glasses is caused by model uncertainty rather than uncertainty in measured data.

With calculated $r_{B}$ and $r_{N a}$ from quenched glasses (Figure 3-17 and Figure 3-18), the calculated $r_{r B}$ and $r_{r N a}$ from most heat-treated glasses underpredict $r_{h B}$ and $r_{h N a}$, but generally less than by an order of magnitude. However, the $r_{h B}$ and $r_{h N a}$ were substantially overpredicted for three glasses (CVS2-33, NPAl-1, and NP-Li-2). For three glasses (CVS1-16, CVS2-35, and CVS2-85), the calculated $r_{r B}$ were significantly lower than measured $r_{h B}$ ( 9 to 14 times less). No such large underprediction occurred in the case of $r_{r N a}$.

With measured $r_{B}$ and $r_{N a}$ from quenched glasses (Figure 3-19 and Figure 3-20), only one glass (NPLi-2) has a substantially overpredicted $r_{h B}$. Four glasses (CVS1-16, CVS2-35, CVS2-85, and NP-Al-2) have significantly underpredicted $r_{h B}$ (10 to 12 times less). This result is satisfactory considering the severe impact (as large as two orders of magnitude) of NP precipitation on PCT (as seen in Figure 3-11), the large uncertainty of the semiquantitative XRD measurement of crystallinity fraction (up to 50\%), and the large uncertainty in the composition of crystalline phases.

A better agreement exists between $r_{r N a}$ and $r_{h N a}$ than between $r_{r B}$ and $r_{h B}$ when measured $r_{B}$ and $r_{N a}$ were used for quenched glasses in Equation (5) (Figure 3-20). Only three glasses have substantially overpredicted (6 to 60 times higher) Na releases $\left(r_{r N a}<r_{h N a}\right)$ : NP-Al-4, NP-B-1, and NP-Li-2. These three 
compositions produced low-SiO ${ }_{2}$ residual glass; moreover, NP-B-1 is a B-free glass, and the anomalous behavior of NP-Li-2 glass is discussed above. The good agreement between $r_{r N a}$ and $r_{h N a}$ indicates that secondary effects and perhaps even uncertainty in XRD data may not have an overwhelming impact on our ability to predict PCT releases from heat-treated glasses. The less successful prediction $r_{h B}$ was probably caused by the inadequacy of the model as represented by coefficients listed in Table 2.1 for some residual glasses whose composition was outside the region for which the models were developed.

Table 3.6. Composition of Glasses Precipitating NP (in mass fractions of oxides)

\begin{tabular}{|c|c|c|c|c|c|c|c|c|c|c|c|}
\hline Glass & $\mathrm{I}_{2} \mathrm{O}_{3}$ & $\mathrm{~B}_{2} \mathrm{O}_{3}$ & $\overline{\mathrm{CaO}}$ & $\mathrm{Fe}_{2} \mathrm{O}_{3}$ & $\mathbf{L i}_{2} \mathbf{O}$ & MgO & $\mathrm{Na}_{2} \mathrm{O}$ & $\mathrm{SiO}_{2}$ & $\mathrm{ZrO}_{2}$ & Others & sum \\
\hline CVS1-16 & & 00500 & 0000 & 0.0858 & 0.0100 & 0.0800 & 0.1873 & 0.4327 & 00000 & & 98 \\
\hline & & 0544 & & 0 & 4 & & 1 & 92 & 0 & & \\
\hline-34 & 388 & 1743 & 00 & 200 & 0.0369 & 00 & 000 & 200 & 000 & 00 & 88 \\
\hline 35 & 340 & 0.0500 & 0 & 632 & 0.0428 & 00 & 000 & 00 & 000 & & 0.1340 \\
\hline & & .1717 & & 00 & 1 & & 0 & & & & \\
\hline-85 & 1700 & 0.0600 & 0.0050 & .0050 & 0.0700 & 0.0200 & 0.1800 & 0.4401 & 0.0050 & 49 & 700 \\
\hline 86 & 700 & .0600 & & 50 & 6 & 50 & 00 & 765 & & & 700 \\
\hline 8 & & 3 & 0 & 0 & 9 & 00 & 0 & 00 & 00 & & \\
\hline-16 & 499 & 0.0004 & 0.0099 & 536 & 0.0225 & 0.0004 & 0.0993 & 0.4204 & 0.0345 & 091 & 0.2499 \\
\hline & & & & & & & & & & & \\
\hline NP-Al-1 & 631 & 0.0870 & 0.0122 & 1081 & 0.0489 & 0.0074 & 0.2205 & 0.4174 & 0.0032 & 22 & 0.0631 \\
\hline $\mathrm{NP}-1$ & 776 & 0.0857 & 20 & 064 & 0.0482 & 073 & 0.2171 & 0.4109 & 31 & & 776 \\
\hline & & & & & & & & & & & \\
\hline NP & 931 & 0.0749 & 105 & 931 & 0.0422 & 0.0064 & 0.1899 & 0.3595 & 0.0027 & 277 & 0.1931 \\
\hline NP-Na-1 & 498 & 0.0871 & 22 & 82 & 0.0490 & 0.0074 & 0.1331 & 0.4178 & 0.0032 & 22 & 0.1498 \\
\hline NP-Na-2 & 472 & 0.0856 & 20 & & 0.0481 & 73 & 0.1482 & 0.4105 & 0.0 & & 172 \\
\hline NP-Si-3 & 222 & 0.0711 & 0.0099 & 383 & 0.0400 & 0.0060 & 0.1801 & 0.4535 & 0.0026 & 263 & 0.1222 \\
\hline NP & 176 & 0.0683 & 96 & 349 & 0.0384 & 058 & 0.1732 & 0.4743 & 0.0025 & 53 & 0.1176 \\
\hline-1 & 497 & 0.0000 & 22 & & 0.1 & 074 & 0.2206 & 0.4176 & 32 & & 497 \\
\hline NP-B-2 & 197 & 0.2002 & 0.0097 & 0.0865 & 0.0392 & 0.0059 & 0.1765 & 0.3340 & 0.0025 & 0.0258 & 0.1197 \\
\hline NP-K-1 & 337 & 0.0777 & 0.0109 & 0.0966 & 0.0437 & 0.0066 & 0.1971 & 0.3730 & 0.0028 & 0.0578 & 0.1337 \\
\hline NP-K-2 & 0.1296 & 0.0753 & 0.0105 & 0.0936 & 0.0424 & 0.0064 & 0.1910 & 0.3615 & 0.0027 & 0.0870 & 0.1296 \\
\hline NP-Li-1 & 0.1442 & 0.0838 & 0.0117 & 0.1042 & 0.0000 & 0.0071 & 0.2125 & 0.4023 & 0.0030 & 0.0310 & 0.1442 \\
\hline NP-Li-2 & 0.1327 & 0.0771 & 0.0108 & 0.0958 & 0.0801 & 0.0066 & 0.1955 & 0.3701 & 0.0028 & 0.0285 & 0.1327 \\
\hline
\end{tabular}


Table 3.7. Crystallinity (in mass fractions of minerals) of HeatTreated Glasses with NP

\begin{tabular}{|c|c|c|c|c|c|c|c|c|}
\hline Glass & Albite & Cristobali & Eucryptite & Gehlinite & e Lithium silicate & NP & Spinel & Sum $_{\text {cr }}$ \\
\hline CVS1-16 & & & & & & 0.170 & & 0.170 \\
\hline CVS2-33 & & & & & & 0.150 & & 0.150 \\
\hline CVS2-34 & & & & & & & & 0.000 \\
\hline CVS2-35 & & 0.020 & & 0.070 & & 0.100 & & 0.190 \\
\hline CVS2-63 & & & & & & 0.150 & & 0.150 \\
\hline CVS2-85 & & & & & 0.210 & 0.330 & & 0.540 \\
\hline CVS2-86 & & & & & & 0.050 & & 0.050 \\
\hline CVS2-98 & & & & & & & & 0.000 \\
\hline CVS3-16 & & & 0.330 & & & 0.400 & & 0.730 \\
\hline NP-BL & 0.017 & & & & & 0.298 & & 0.298 \\
\hline NP-Al-1 & & & & & & & & 0.000 \\
\hline NP-Al-2 & & & & & & 0.050 & & 0.050 \\
\hline NP-Al-3 & 0.022 & & & & & 0.384 & & 0.384 \\
\hline NP-Al-4 & 0.050 & & & & & 0.402 & & 0.402 \\
\hline NP-Na-1 & & 0.010 & & & & 0.187 & 0.002 & 0.199 \\
\hline NP-Na-2 & & 0.003 & & & & 0.323 & 0.002 & 0.328 \\
\hline NP-Si-3 & & & & & & & & 0.000 \\
\hline NP-Si-4 & & & & & & & & 0.000 \\
\hline NP-B-1 & & & & & & 0.328 & & 0.328 \\
\hline NP-B-2 & & & & & & & & 0.000 \\
\hline NP-K-1 & & & & & & 0.293 & & 0.293 \\
\hline NP-K-2 & & & & & & 0.284 & & 0.284 \\
\hline NP-Li-1 & & & & & & 0.010 & & 0.010 \\
\hline NP-Li-2 & & & & & 0.238 & 0.291 & & 0.529 \\
\hline
\end{tabular}

1 vol\% of an unidentified crystalline phase was detected by XRD in NP-Si-3 and NP-Si-4. 
Table 3.8. Residual Glass Composition (in mass fractions of oxides): Glasses Precipitating NP 1

\begin{tabular}{|c|c|c|c|c|c|c|c|c|c|c|}
\hline & & $\mathbf{O}_{3}$ & & ${ }_{2} \mathrm{O}_{3}$ & $\mathrm{i}_{2} \mathrm{O}$ & & 0 & 2 & & \\
\hline & & & & & & & & & & \\
\hline & & & & & & & & & & \\
\hline & & & & & & & & 0 & & \\
\hline & & & & & & & & & & \\
\hline & & & & & & & & & & \\
\hline & & & & & & & & & & \\
\hline & & & & & & & & & & \\
\hline & & & & & & & & & & \\
\hline & & & & & & & & & & \\
\hline & & & & & & & & & & \\
\hline P-Al-1 & & 0.0870 & & & & & & 0 & & \\
\hline $1-2$ & 8 & 0902 & & 0 & 7 & 7 & & ( & & 334 \\
\hline -Al-3 & 9 & 0.1286 & & 7 & 3 & & & 0 & & 176 \\
\hline -Al-4 & 13 & 67 & & 99 & 69 & & & 30 & & 0 \\
\hline -Na-1 & 32 & 87 & & 34 & 12 & & & 0.4104 & 39 & 94 \\
\hline -Na-2 & 465 & 73 & & & 16 & & & 0.4032 & 46 & 62 \\
\hline 3 & 2 & 1 & & 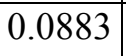 & 0 & 0 & & & 026 & \\
\hline & & & & & & & & & & \\
\hline & & & & & & & & 0 & & \\
\hline 2 & & 02 & 97 & 65 & 392 & 59 & 65 & 0 . & 0 & 2Jo \\
\hline-1 & & 00 & & 66 & & & & 0.3523 & & \\
\hline & & & & & & & & 1 & & \\
\hline & & & & & & & & & & \\
\hline & & & & & & & & & & \\
\hline
\end{tabular}


Table 3.9. Residual Glass Composition (in mass fractions of oxides): Glasses Precipitating NP 2

\begin{tabular}{|c|c|c|c|c|c|c|c|c|c|c|}
\hline & & ${ }_{2} \mathrm{O}_{3}$ & & $e_{2} \mathbf{O}_{3}$ & 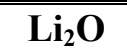 & & $\mathrm{Na}_{2} \mathbf{O}$ & $2_{2}$ & )$_{2}$ & \\
\hline & & & & & & & & & & \\
\hline & & & & & & & & & & \\
\hline & & & & & & & & & & \\
\hline & & & & & & & & & & \\
\hline & & & & & & & & & & \\
\hline & & & & & & & & & & \\
\hline & & 2 & & & 3 & & & 51 & & \\
\hline & & & & & & & & & & \\
\hline & & & & & & & & & & \\
\hline & & & & & & & & & & \\
\hline & & & & & & & & & & \\
\hline & & & & & & & & 50 & & \\
\hline & & & & & & & & & & \\
\hline & & & & & & & & & & \\
\hline & & & & & & & & & & \\
\hline & & & & & & & & 42 & & \\
\hline & & & & & & & & & & \\
\hline & & & & & & & & & & \\
\hline & & & & & & & & & & \\
\hline & & & & & & & & & & \\
\hline & & & & & & & & & 40 & \\
\hline & & & & & & & & 49 & & 199 \\
\hline & & & & & & & & & & 313 \\
\hline $\mathrm{i}-2$ & 74 & 38 & 29 & & 22 & 39 & & 369 & 059 & 0.058 \\
\hline
\end{tabular}


Table 3.10. Measured $r_{B}$ and $r_{N a}$ (in $\mathrm{g} / \mathrm{m}^{2}$ ) from Quenched and Heat-Treated Glasses with NP

\begin{tabular}{|l|c|c|c|c||}
\hline Glass & $\mathbf{Q}, \mathbf{M}$ & $\mathbf{Q}, \mathbf{M}$ & $\mathbf{H}, \mathbf{M}$ & $\mathbf{H}, \mathbf{M}$ \\
\hline & $\boldsymbol{r}_{\boldsymbol{B}}$ & $\boldsymbol{r}_{\boldsymbol{N} \boldsymbol{a}}$ & $\boldsymbol{r}_{\boldsymbol{h} \boldsymbol{B}}$ & $\boldsymbol{r}_{\boldsymbol{h} \boldsymbol{a} \boldsymbol{a}}$ \\
\hline CVS1-16 & 0.52 & 0.54 & 22.83 & 8.85 \\
\hline CVS2-33 & 1.72 & 1.61 & 11.53 & 7.41 \\
\hline CVS2-34 & 4.34 & 2.70 & 4.52 & 2.59 \\
\hline CVS2-35 & 0.32 & 0.91 & 23.18 & 10.38 \\
\hline CVS2-63 & 0.34 & 0.39 & 3.65 & 2.16 \\
\hline CVS2-85 & 0.46 & 0.75 & 45.55 & 18.34 \\
\hline CVS2-86 & 0.12 & 0.26 & 0.27 & 0.3 \\
\hline CVS2-98 & 4.52 & 2.80 & 2.97 & 2.76 \\
\hline CVS3-16 & 0.27 & 0.08 & 1.25 & 0.04 \\
\hline NP-BL & 0.54 & 0.78 & 42.81 & 20.81 \\
\hline NP-A1-1 & 1.64 & 1.79 & 1.73 & 1.87 \\
\hline NP-A1-2 & 0.66 & 1.44 & 9.1 & 6.59 \\
\hline NP-A1-3 & 0.38 & 0.61 & 45.82 & 17.18 \\
\hline NP-A1-4 & 0.37 & 0.60 & 44.94 & 15.41 \\
\hline NP-Na-1 & - & - & 1.6 & 0.4 \\
\hline NP-Na-2 & - & - & 22.04 & 5.97 \\
\hline NP-Si-3 & 0.36 & 0.49 & 0.36 & 0.49 \\
\hline NP-Si-4 & 0.28 & 0.41 & 0.28 & 0.41 \\
\hline NP-B-1 & - & 1.90 & - & 1.17 \\
\hline NP-B-2 & 4.31 & 2.90 & - & - \\
\hline NP-K-1 & 1.06 & 1.23 & 48.19 & 27.06 \\
\hline NP-K-2 & 1.76 & 1.81 & 45.22 & 28.01 \\
\hline NP-Li-1 & 0.29 & 0.43 & 0.76 & 0.59 \\
\hline NP-Li-2 & 1.13 & 1.56 & 1.78 & 1.54 \\
\hline & & & &
\end{tabular}


Table 3.11. Calculated $r_{B}$ and $r_{N a}$ (in $\mathrm{g} / \mathrm{m}^{2}$ ) from Quenched and Heat-Treated Glasses with NP

\begin{tabular}{|c|c|c|c|c|c|c|}
\hline Glass & $\mathbf{Q}, \mathbf{C}$ & $\mathbf{Q}, \mathbf{C}$ & $\mathrm{H}, \mathrm{QC}, \mathbf{R C}$ & $\mathbf{H}, \mathbf{Q C}, \mathbf{R C}$ & H, QM, RC & H, QM, RC \\
\hline & $r_{B}$ & $r_{N a}$ & $\boldsymbol{r}_{r B}$ & $\boldsymbol{r}_{r N a}$ & $\boldsymbol{r}_{r B}$ & $r_{r N a}$ \\
\hline & & & \multicolumn{2}{|c|}{ with $r_{j}$ calculated } & \multicolumn{2}{|c|}{ with $r_{j}$ measured } \\
\hline CVS1-16 & 0.44 & 0.50 & 1.61 & 1.73 & 1.95 & 1.90 \\
\hline CVS2-33 & 5.69 & 5.83 & 27.12 & 26.26 & 6.63 & 5.77 \\
\hline CVS2-34 & 2.83 & 2.13 & 2.83 & 2.13 & 4.34 & 2.70 \\
\hline CVS2-35 & 0.36 & 0.60 & 2.51 & 3.59 & 2.19 & 6.03 \\
\hline CVS2-63 & 0.25 & 0.41 & 0.69 & 1.16 & 0.97 & 1.09 \\
\hline CVS2-85 & 0.53 & 0.47 & 4.96 & 5.28 & 3.67 & 14.47 \\
\hline CVS2-86 & 0.08 & 0.11 & 0.10 & 0.14 & 0.16 & 0.34 \\
\hline CVS2-98 & 2.83 & 2.13 & 2.83 & 2.13 & 4.52 & 2.80 \\
\hline CVS3-16 & $1.50 \mathrm{E}-03$ & $1.40 \mathrm{E}-03$ & $1.00 \mathrm{E}-02$ & $1.00 \mathrm{E}-02$ & $1.62 \mathrm{E}+06$ & $1.52 \mathrm{E}+04$ \\
\hline NP-BL & 1.02 & 0.95 & 26.35 & 20.33 & 10.35 & 15.16 \\
\hline NP-Al-1 & 9.26 & 8.59 & 9.26 & 8.59 & 1.64 & 1.79 \\
\hline NP-Al-2 & 6.03 & 5.59 & 9.63 & 8.74 & 0.94 & 2.10 \\
\hline NP-Al-3 & 0.32 & 0.30 & 18.07 & 12.78 & 23.87 & 42.07 \\
\hline NP-Al-4 & 0.20 & 0.19 & 16.62 & 11.22 & 50.96 & 94.15 \\
\hline NP-Na-1 & 0.22 & 0.17 & 0.84 & 0.58 & - & - \\
\hline NP-Na-2 & 0.31 & 0.25 & 5.38 & 3.39 & - & - \\
\hline NP-Si-3 & 0.63 & 0.58 & 0.63 & 0.58 & 0.36 & 0.49 \\
\hline NP-Si-4 & 0.54 & 0.50 & 0.54 & 0.50 & 0.28 & 0.41 \\
\hline NP-B-1 & 0.36 & 0.42 & 7.05 & 7.41 & - & 70.41 \\
\hline NP-B-2 & 4.89 & 3.28 & 4.89 & 3.28 & 4.31 & 2.90 \\
\hline NP-K-1 & 1.03 & 0.94 & 19.78 & 15.01 & 20.65 & 22.07 \\
\hline NP-K-2 & 1.03 & 0.92 & 17.53 & 12.99 & 36.91 & 33.44 \\
\hline NP-Li-1 & 0.35 & 0.39 & 0.38 & 0.41 & 0.31 & 0.46 \\
\hline NP-Li-2 & 2.34 & 1.92 & 47.70 & 46.95 & 10.13 & 30.06 \\
\hline
\end{tabular}




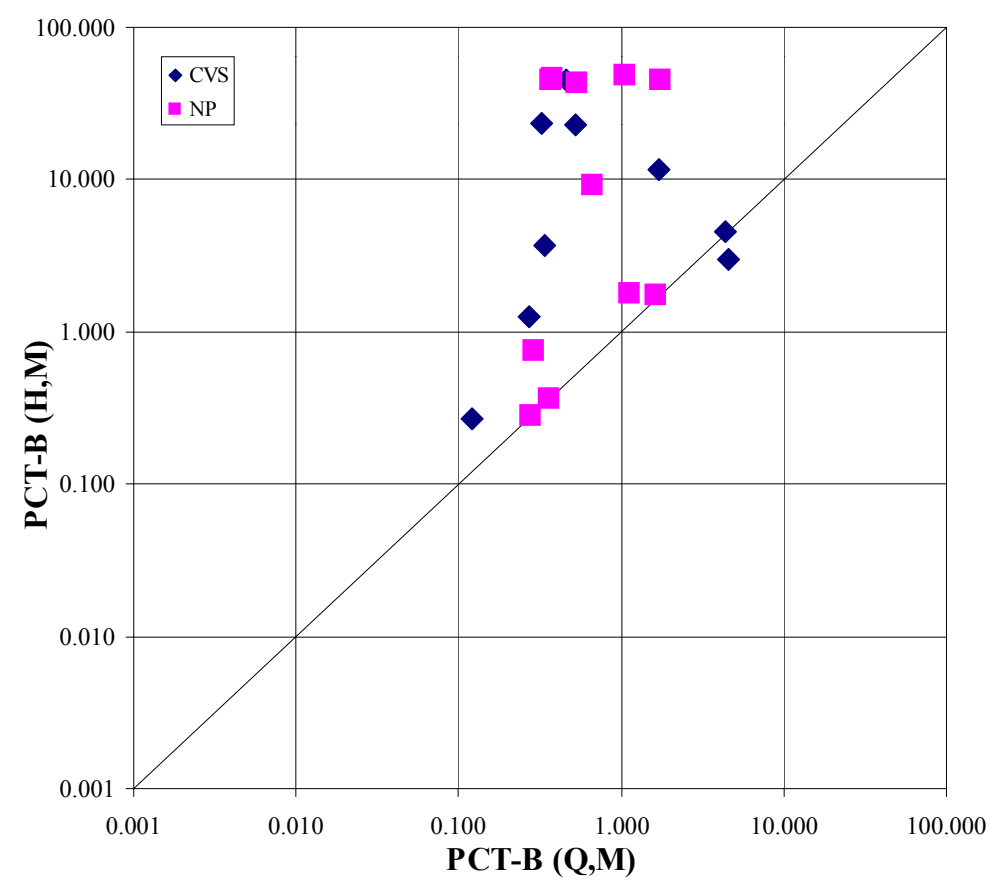

Figure 3-11. Glasses Precipitating NP: $r_{h B}$ vs. $r_{B}$

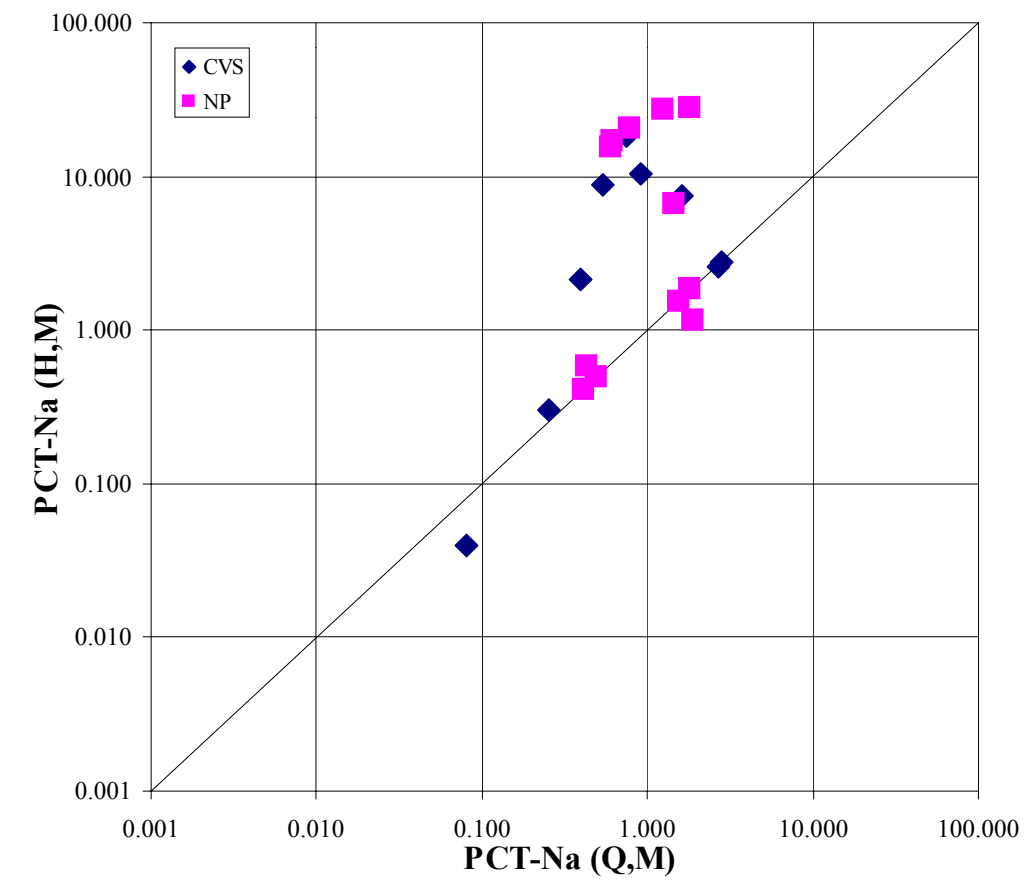

Figure 3-12. Glasses Precipitating NP: $r_{h N a}$ vs. $r_{\mathrm{Na}}$ 


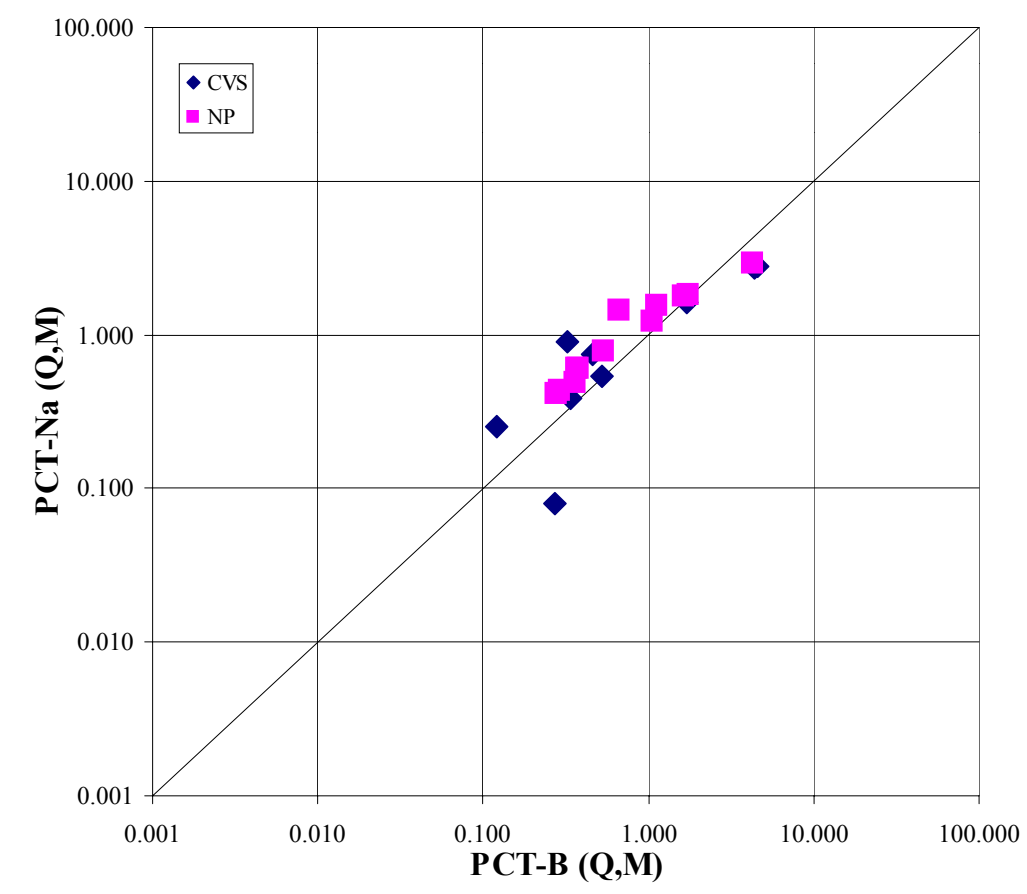

Figure 3-13. Glasses Precipitating NP: $r_{N a}$ vs. $r_{B}$

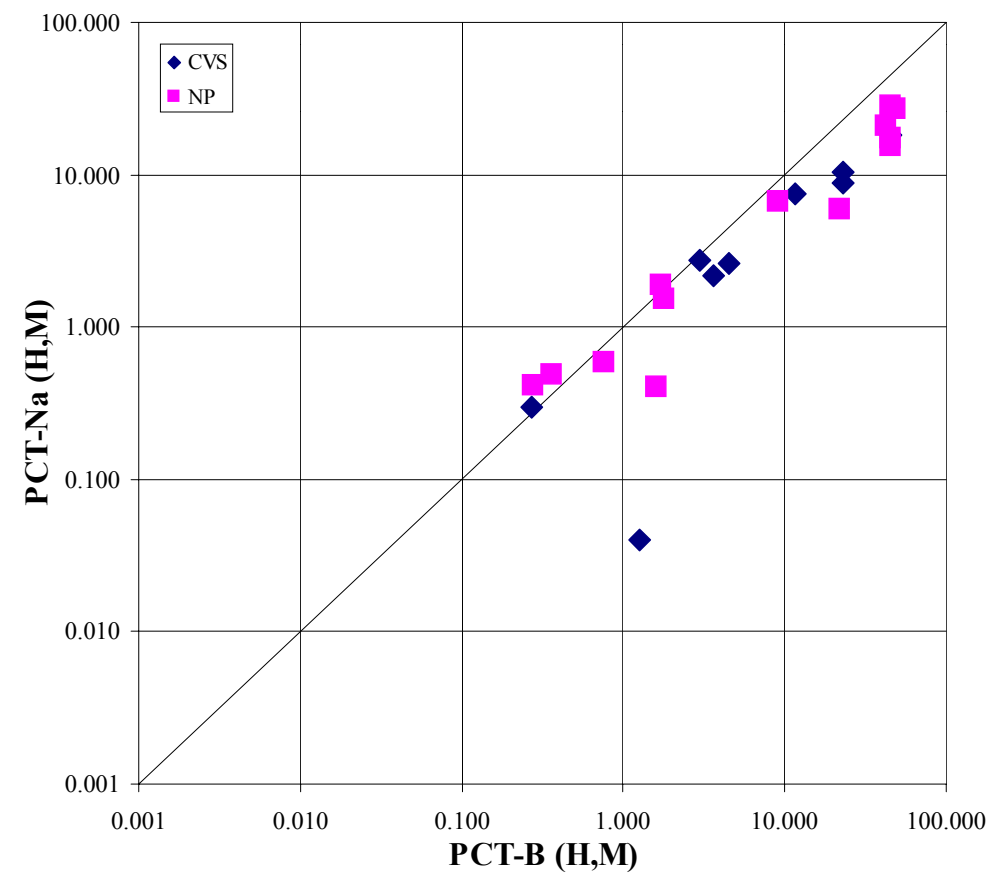

Figure 3-14. Glasses Precipitating NP: $r_{h N a}$ vs. $r_{h B}$ 


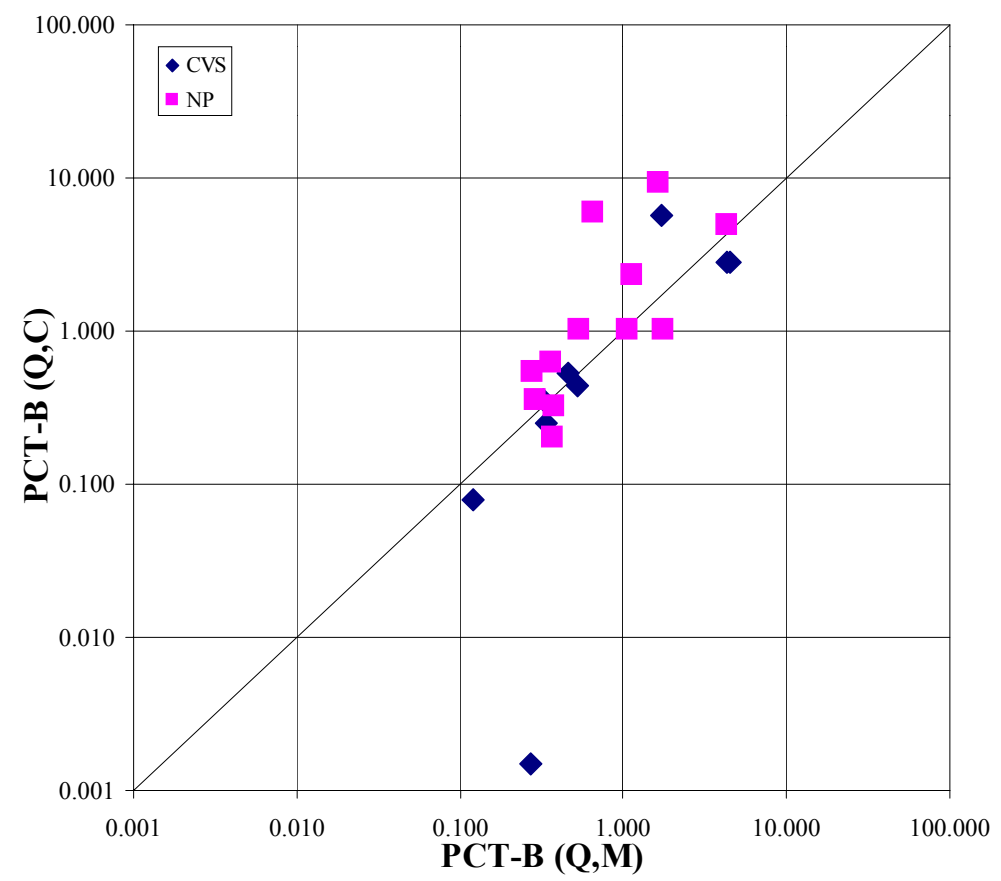

Figure 3-15. Glasses Precipitating NP: $r_{B}$, Calculated vs. Measured

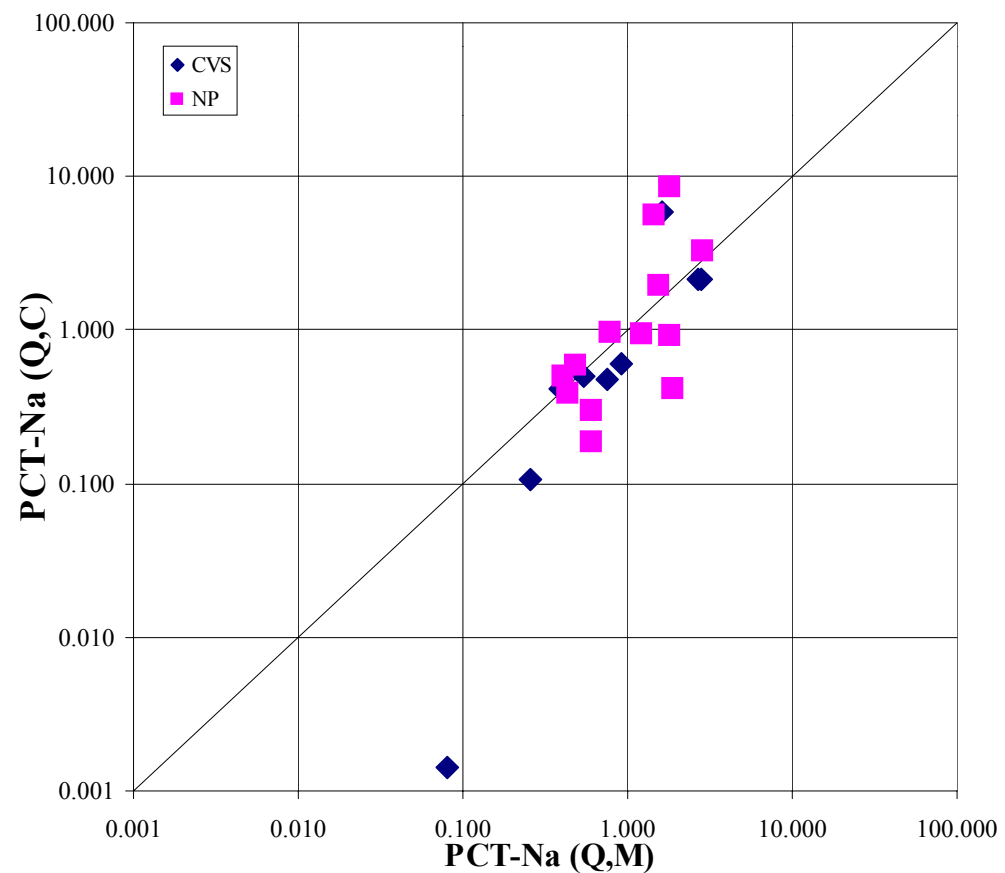

Figure 3-16. Glasses Precipitating NP: $r_{\mathrm{Na}}$, Calculated vs. Measured 


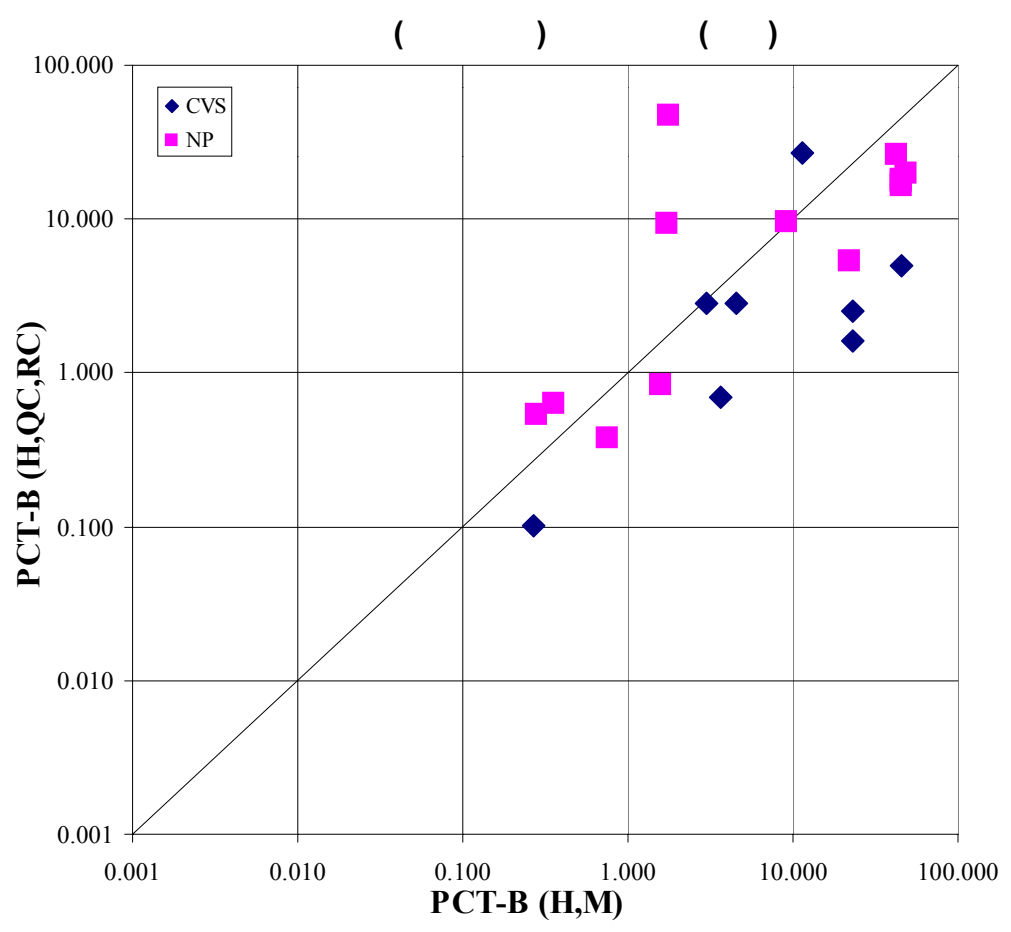

Figure 3-17. Glasses Precipitating NP: $r_{r B}$ (calculated) vs. $r_{h B}$ (measured)

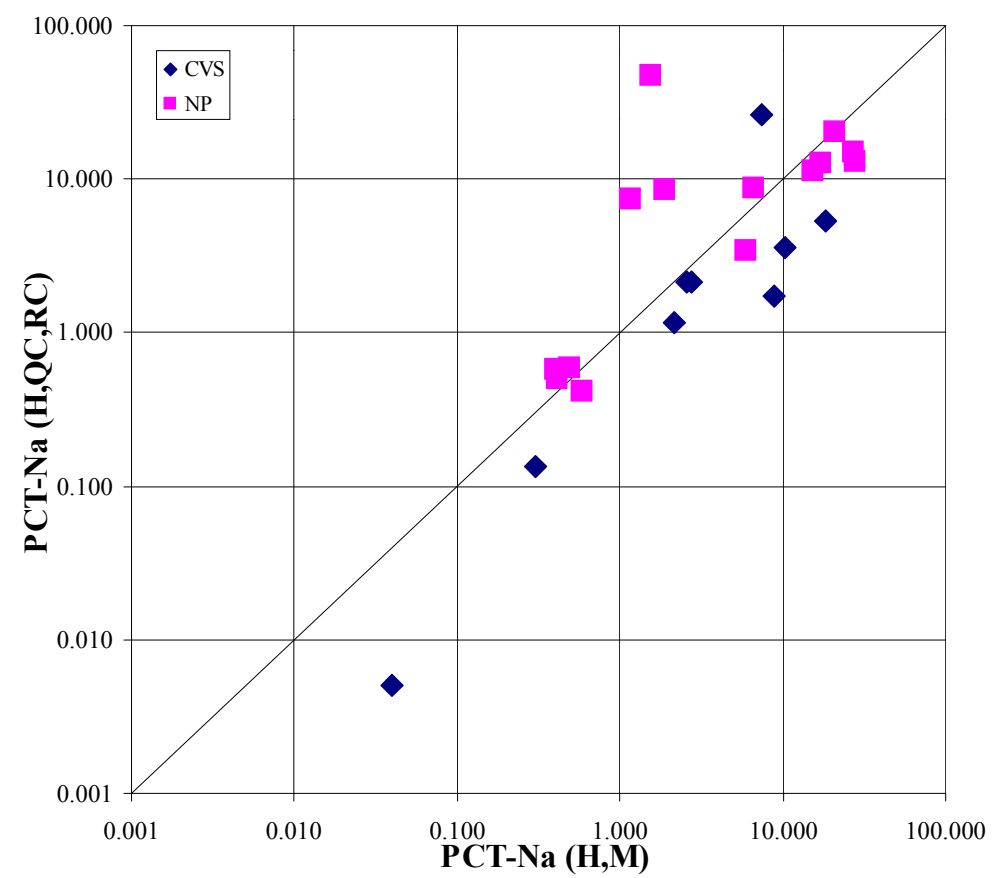

Figure 3-18. Glasses Precipitating NP: $r_{r N a}$ (calculated) vs. $r_{h N a}$ 
(measured) 


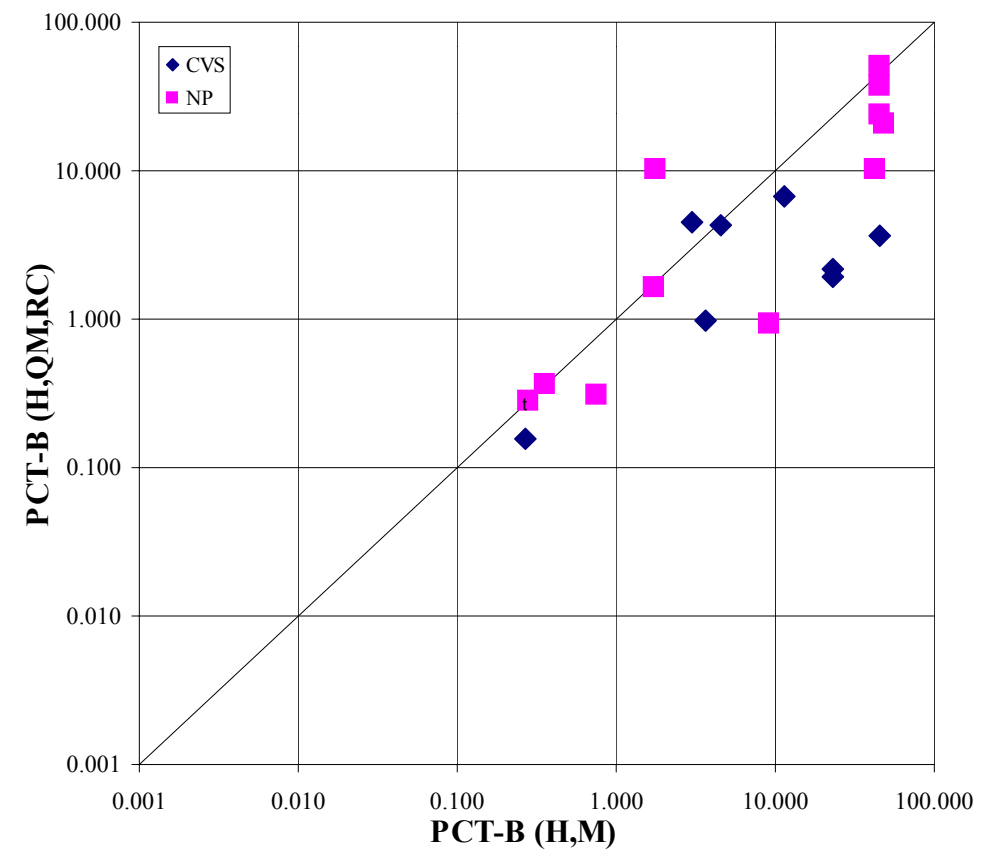

Figure 3-19. Glasses Precipitating NP: $r_{x B}$ (calculated using measured $r_{B}$ ) vs. $r_{h B}$ (measured)

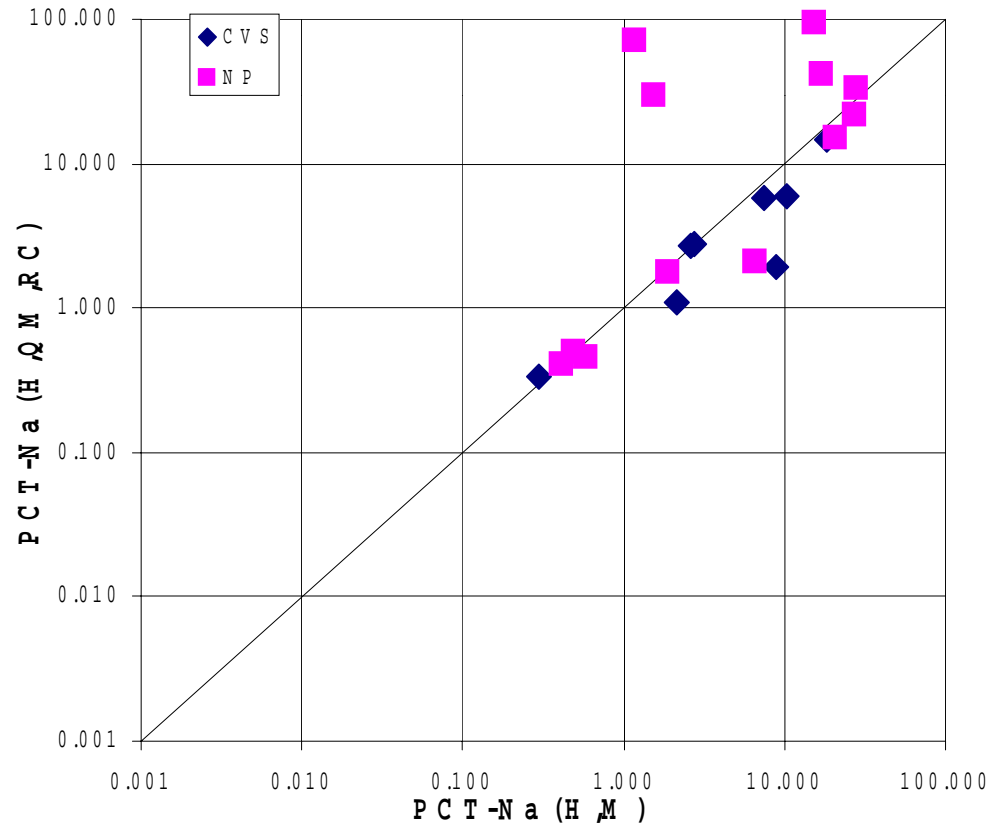

Figure 3-20. Glasses Precipitating NP: $r_{r N a}$ (calculated using measured $\left.r_{N a}\right)$

vs. $r_{h N a}$ (measured) 


\subsection{Glasses Precipitating other Crystalline Phases}

Some glasses precipitated major crystalline phases other than clinopyroxene or NP upon CCC or IHT. The compositions of these glasses are listed in Table 3.12 to Table 3.15. Table 3.12 contains a glass, CVS2-70, with $\mathrm{Al}_{2} \mathrm{O}_{3}$ exceeding the 0.17 limit listed in Table 2.2. Two high-temperature glasses melting above $1200^{\circ} \mathrm{C}, \mathrm{CVS} 3-20$ and CVS3-21, are also outside the composition region shown in Table 2.2. These glasses have zero B content and somewhat higher content of Others. In addition, CVS3-20 has a high $\mathrm{Zr}$ content.

Most glasses listed in Table 3.12 to Table 3.15 precipitated more than one crystalline phase, as seen in Table 3.16 to Table 3.19, that classify the glasses into four groups: glasses with lithium silicate, glasses with cristobalite, glasses with hematite, and glasses with zircon. The classification is somewhat fuzzy, allowing some glasses to belong to more than one category. Two glasses, CVS2-78 and CVS2-80, precipitated large fractions of both cristobalite and zircon and are listed in both tables. Similarly, the CVS2-79 glass precipitated a large fraction of cristobalite and hematite and was listed in two tables. Some glasses that precipitated substantial fractions of lithium-silicate and zircon are dealt with in Section 3-1 (CVS1-8, 1-15, 1-22, 2-25, 2-36 and, 2-42) and Section 3-2 (NP-Li-2). These glasses are not included in this section. Two glasses, CVS1-18 and CVS1-6, precipitated baddeleyite.

Residual glass compositions are listed in Table 3.20 to Table 3.23. When the quenched glass composition was at the boundary of the composition region defined in Table 2.2, crystallization often resulted in residual glasses positioned slightly outside the boundary. We believe that the component coefficients listed in Table 2.1 are still applicable to these glasses. One residual glass, CVS2-70, had over 20 mass $\% \mathrm{Al}_{2} \mathrm{O}_{3}, 3$ mass $\%$ above the upper limit. The original composition of this glass was already above the upper limit in $\mathrm{Al}_{2} \mathrm{O}_{3}$ content.

Table 3.24 to Table 3.27 list and Figure 3-21 to Figure 3-24 display the measured $r_{B}$ and $r_{N a}$ for each group of glasses. As Figure 3-21 and Figure 3-22 show, CCC increased $r_{B}$ and $r_{N a}$ by more than an order of magnitude for five glasses: CVS2-68, CVS2-70, CVS2-78, CVS2-79, and CVS2-80. Three of these glasses, CVS2-78, CVS2-79, and CVS2-80, precipitated cristobalite (15 to 22 mass\%) plus 3 to 9 mass\% zircon or 1 mass $\%$ hematite. The remaining two glasses (CVS2-68, CVS2-70) precipitated 8 to 10 mass $\%$ lithium silicate. Interestingly, lithium silicate appears to have a negative effect on glass durability, which is at variance with its calculated effects shown in Table 2.3. Moreover, glasses that precipitated $\leq 7 \mathrm{mass} \%$ lithium silicates did not exhibit any major change in $r_{B}$ and $r_{N a}$ after CCC-treatment.

From quenched glasses, $r_{N a}$ values appear somewhat lower than $r_{B}$ values (Figure 3-23). This is as expected because $\mathrm{Na}$ can form or be absorbed on secondary mineral phases that precipitate from the solution, whereas B does not participate in secondary reaction products under PCT conditions. ${ }^{\text {a }}$ For CCC

\footnotetext{
${ }^{a}$ No obvious relationship exists between minerals that precipitate from glass and those that precipitate from aqueous medium upon corrosion.
} 
glasses, the comparison of $r_{h B}$ and $r_{h N a}$ reveals a more complex behavior (Figure 3-24). CVS1-18 glass stands out: the $r_{h N a}$ from this glass is nearly twice as high as the $r_{h B}$.

Figure 3-25 and Figure 3-26 compare measured and calculated of $r_{h B}$ and $r_{h N a}$ from quenched glasses (see also Table 3.28 to Table 3.31). Considering that some compositions are on or outside the boundary of the composition region on which the PCT coefficients were validated, agreement between calculated and measured values appears reasonable.

Figure 3-27 to Figure 3-30 clearly show that $r_{h B}$ and $r_{h N a}$ were grossly underpredicted for several compositions, whether measured or calculated of $r_{B}$ and $r_{N a}$ were used. Five glasses stand out: CVS2-68, CVS2-70, CVS2-78, CVS2-79, and CVS2-80 (see also Table 3.28 to Table 3.31), the same glasses whose releases were strongly affected by the CCC treatment.

Three of these glasses, CVS2-78, CVS2-79, and CVS2-80, precipitated large fractions of cristobalite. These three glasses had a low content of alkalis and thus were prone to phase separation. It is likely that these glasses separated into a continuous low-silica phase and a dispersed high-silica phase that subsequently crystallized, forming cristobalite. It is conceivable that the dispersed phase contained silica that did not crystallize and hence the continuous phase was more impoverished of silica than estimated in Table 3.21. This may explain why the calculated $r_{r B}$ and $r_{r N a}$, though several times higher than $r_{B}$ and $r_{N a}$, were still not high enough to match the measured values.

CVS2-68 and CVS2-70 glasses precipitated lithium silicate. Calculations yield $r_{r B}<r_{B}$ and $r_{r N a}<r_{N a}$ from glasses with lithium silicate (compare the values in Table 3.24 and Table 3.28), but measurements revealed the opposite effect $\left(r_{h B}>r_{B}\right.$ and $\left.r_{h N a}>r_{N a}\right)$. This discrepancy between the measured and calculated values is seen in Figure 3-27 to Figure 3-30. The measured results indicate that the precipitation of large fractions of lithium silicate produces secondary effects with deteriorating impact on glass durability. However, such strong secondary effects are unlikely. Alternatively, it is possible that the precipitation of lithium silicate could decrease the alkali content in the residual glass to the point at which phase separation occurred, decreasing its silica content and thus leading to an inferior product. Such a decrease would have to be localized around the crystals because the average residual-glass composition is outside the immiscibility region.

Table 3.12. Composition of Glasses Precipitating Lithium-Silicates

\begin{tabular}{|l|c|c|c|c|c|c|c|c|c|c|c|}
\hline \hline Glass & $\mathbf{A l}_{2} \mathbf{O}_{3}$ & $\mathbf{B}_{2} \mathbf{O}_{3}$ & $\mathbf{C a O}$ & $\mathbf{F e}_{2} \mathbf{O}_{3}$ & $\mathbf{L i}_{2} \mathbf{O}$ & $\mathbf{M g O}$ & $\mathbf{N a}_{2} \mathbf{O}$ & $\mathbf{S i O}_{2}$ & $\mathbf{Z r O}_{2}$ & Others & Sum \\
\hline CVS1-5 & 0.080 & 0.050 & 0.000 & 0.150 & 0.070 & 0.000 & 0.070 & 0.570 & 0.000 & 0.010 & 1.000 \\
\hline CVS1-18 & 0.000 & 0.050 & 0.020 & 0.020 & 0.070 & 0.080 & 0.119 & 0.422 & 0.130 & 0.090 & 1.000 \\
\hline CVS2-22 & 0.037 & 0.050 & 0.000 & 0.020 & 0.070 & 0.080 & 0.074 & 0.570 & 0.000 & 0.100 & 1.000 \\
\hline CVS2-39 & 0.026 & 0.050 & 0.000 & 0.074 & 0.070 & 0.080 & 0.136 & 0.484 & 0.070 & 0.010 & 1.000 \\
\hline CVS2-68 & 0.164 & 0.136 & 0.001 & 0.005 & 0.070 & 0.000 & 0.080 & 0.504 & 0.000 & 0.041 & 1.000 \\
\hline CVS2-70 & 0.182 & 0.142 & 0.001 & 0.008 & 0.069 & 0.001 & 0.081 & 0.486 & 0.001 & 0.030 & 1.000 \\
\hline CVS2-76 & 0.020 & 0.060 & 0.040 & 0.105 & 0.063 & 0.005 & 0.180 & 0.502 & 0.005 & 0.020 & 1.000 \\
\hline
\end{tabular}




\begin{tabular}{|l|c|c|c|c|c|c|c|c|c|c|c||}
\hline Glass & $\mathbf{A l}_{2} \mathbf{O}_{\mathbf{3}}$ & $\mathbf{B}_{\mathbf{2}} \mathbf{O}_{\mathbf{3}}$ & $\mathbf{C a O}$ & $\mathbf{F e}_{2} \mathbf{O}_{3}$ & $\mathbf{L i}_{2} \mathbf{O}$ & $\mathbf{M g O}$ & $\mathbf{N a}_{2} \mathbf{O}$ & $\mathbf{S i O}_{2}$ & $\mathbf{Z r O}_{2}$ & Others & Sum \\
\hline CVS2-81 & 0.020 & 0.060 & 0.005 & 0.105 & 0.070 & 0.040 & 0.174 & 0.440 & 0.005 & 0.081 & 1.000 \\
\hline CVS2-82 & 0.020 & 0.095 & 0.005 & 0.005 & 0.070 & 0.040 & 0.180 & 0.560 & 0.005 & 0.020 & 1.000 \\
\hline CVS2-84 & 0.020 & 0.060 & 0.005 & 0.105 & 0.070 & 0.005 & 0.180 & 0.455 & 0.080 & 0.020 & 1.000 \\
\hline CVS2-90 & 0.020 & 0.160 & 0.005 & 0.101 & 0.070 & 0.005 & 0.054 & 0.560 & 0.005 & 0.020 & 1.000 \\
\hline
\end{tabular}

Table 3.13. Composition of Glasses Precipitating Cristobalite

\begin{tabular}{|l|l|l|l|l|l|l|l|l|l|l|l|}
\hline Glass & $\mathbf{A l}_{2} \mathbf{O}_{\mathbf{3}}$ & $\mathbf{B}_{\mathbf{2}} \mathbf{O}_{\mathbf{3}}$ & $\mathbf{C a O}$ & $\mathbf{F e}_{2} \mathbf{O}_{\mathbf{3}}$ & $\mathbf{L i}_{2} \mathbf{O}$ & $\mathbf{M g O}$ & $\mathbf{N a}_{2} \mathbf{O}$ & $\mathbf{S i O}_{2}$ & $\mathbf{Z r O}_{2}$ & Others & Sum \\
\hline CVS1-9 & 0.000 & 0.196 & 0.000 & 0.140 & 0.010 & 0.080 & 0.054 & 0.420 & 0.000 & 0.100 & 1.000 \\
\hline CVS2-30 & 0.046 & 0.200 & 0.000 & 0.132 & 0.010 & 0.000 & 0.083 & 0.519 & 0.000 & 0.010 & 1.000 \\
\hline CVS2-77 & 0.020 & 0.060 & 0.005 & 0.005 & 0.070 & 0.005 & 0.180 & 0.455 & 0.110 & 0.090 & 1.000 \\
\hline CVS2-78 & 0.020 & 0.160 & 0.005 & 0.070 & 0.025 & 0.040 & 0.050 & 0.560 & 0.050 & 0.020 & 1.000 \\
\hline CVS2-79 & 0.020 & 0.160 & 0.005 & 0.105 & 0.012 & 0.005 & 0.050 & 0.548 & 0.005 & 0.090 & 1.000 \\
\hline CVS2-80 & 0.020 & 0.160 & 0.005 & 0.105 & 0.018 & 0.040 & 0.050 & 0.507 & 0.075 & 0.020 & 1.000 \\
\hline CVS2-90 & 0.020 & 0.160 & 0.005 & 0.101 & 0.070 & 0.005 & 0.054 & 0.560 & 0.005 & 0.020 & 1.000 \\
\hline CVS2-91 & 0.020 & 0.160 & 0.005 & 0.005 & 0.010 & 0.040 & 0.105 & 0.560 & 0.005 & 0.090 & 1.000 \\
\hline
\end{tabular}

Table 3.14. Composition of Glasses Precipitating Hematite

\begin{tabular}{|l|c|c|c|c|c|c|c|c|c|c|c|}
\hline Glass & $\mathbf{A l}_{\mathbf{2}} \mathbf{O}_{\mathbf{3}}$ & $\mathbf{B}_{\mathbf{2}} \mathbf{O}_{\mathbf{3}}$ & $\mathbf{C a O}$ & $\mathbf{F e}_{2} \mathbf{O}_{\mathbf{3}}$ & $\mathbf{L i}_{\mathbf{2}} \mathbf{O}$ & $\mathbf{M g O}$ & $\mathbf{N a}_{2} \mathbf{O}$ & $\mathbf{S i O}_{2}$ & $\mathbf{Z r O}_{2}$ & Others & Sum \\
\hline CVS1-12 & 0.000 & 0.176 & 0.100 & 0.150 & 0.070 & 0.000 & 0.074 & 0.420 & 0.000 & 0.010 & 1.000 \\
\hline CVS2-30 & 0.046 & 0.200 & 0.000 & 0.132 & 0.010 & 0.000 & 0.083 & 0.519 & 0.000 & 0.010 & 1.000 \\
\hline CVS2-79 & 0.020 & 0.160 & 0.005 & 0.105 & 0.012 & 0.005 & 0.050 & 0.548 & 0.005 & 0.090 & 1.000 \\
\hline
\end{tabular}

Table 3.15. Composition of Glasses Precipitating Zircon

\begin{tabular}{|l|c|c|c|c|c|c|c|c|c|c|c|}
\hline \hline Glass & $\mathbf{A l}_{2} \mathbf{O}_{\mathbf{3}}$ & $\mathbf{B}_{2} \mathbf{O}_{\mathbf{3}}$ & $\mathbf{C a O}$ & $\mathbf{F e}_{2} \mathbf{O}_{3}$ & $\mathbf{L i}_{2} \mathbf{O}$ & $\mathbf{M g O}$ & $\mathbf{N a}_{2} \mathbf{O}$ & $\mathbf{S i O}_{2}$ & $\mathbf{Z r O}_{2}$ & Others & Sum \\
\hline CVS1-6 & 0.000 & 0.200 & 0.000 & 0.020 & 0.070 & 0.000 & 0.050 & 0.440 & 0.120 & 0.100 & 1.000 \\
\hline CVS2-78 & 0.020 & 0.160 & 0.005 & 0.070 & 0.025 & 0.040 & 0.050 & 0.560 & 0.050 & 0.020 & 1.000 \\
\hline CVS2-80 & 0.020 & 0.160 & 0.005 & 0.105 & 0.018 & 0.040 & 0.050 & 0.507 & 0.075 & 0.020 & 1.000 \\
\hline CVS3-20 & 0.043 & 0.000 & 0.011 & 0.060 & 0.025 & 0.000 & 0.111 & 0.468 & 0.160 & 0.122 & 1.000 \\
\hline CVS3-21 & 0.045 & 0.000 & 0.012 & 0.063 & 0.026 & 0.000 & 0.116 & 0.491 & 0.120 & 0.127 & 1.000 \\
\hline
\end{tabular}


Table 3.16. Crystallinity (in mass fractions of minerals) of HeatTreated Glasses

with Lithium Silicate

\begin{tabular}{|l|l|l|l|l|l|l|l|l||}
\hline Glass & Baddeleyite & Cristobalite & Enstatite & Eskolaite & Li silicates & Trevorite & Others & Sum \\
\hline CVS1-5 & & & & & 0.01 & $0.03(\mathrm{a})$ & & 0.05 \\
\hline CVS1-18 & 0.13 & & & & 0.03 & & & 0.15 \\
\hline CVS2-22 & & & 0.07 & & 0.07 & & & 0.14 \\
\hline CVS2-39 & & & & & 0.07 & & 0.03 & 0.10 \\
\hline CVS2-68 & & & & 0.02 & 0.08 & & & 0.10 \\
\hline CVS2-70 & & & & 0.01 & 0.10 & & & 0.11 \\
\hline CVS2-76 & & & & & 0.05 & & & 0.05 \\
\hline CVS2-81 & & & & & 0.04 & 0.05 & & 0.09 \\
\hline CVS2-82 & & & & & 0.04 & & & 0.04 \\
\hline CVS2-84 & & & & & 0.05 & & & 0.05 \\
\hline CVS2-90 & & 0.05 & & & 0.01 & 0.01 & & 0.07 \\
\hline
\end{tabular}

(a) The reported fraction of spinel was 0.04 . The fraction of trevorite was estimated at 0.03 to avoid negative Others in the residual glass.

Table 3.17. Crystallinity (in mass fractions of minerals) of HeatTreated Glasses with Cristobalite

\begin{tabular}{|l|c|c|c|c|c|c|}
\hline Glass & Cristobalite & Hematite & Li silicates & Trevorite & Zircon & Sum \\
\hline CVS1-9 & 0.01 & & & 0.07 & & 0.08 \\
\hline CVS2-30 & 0.02 & 0.09 & & & & 0.11 \\
\hline CVS2-77 & 0.02 & & & & & 0.02 \\
\hline CVS2-78 & 0.22 & & & & 0.03 & 0.25 \\
\hline CVS2-79 & 0.20 & 0.01 & & 0.05 & & 0.26 \\
\hline CVS2-80 & 0.15 & & & & 0.09 & 0.24 \\
\hline CVS2-90 & 0.05 & & 0.01 & 0.01 & & 0.07 \\
\hline CVS2-91 & 0.01 & & & & & 0.01 \\
\hline
\end{tabular}

Table 3.18. Crystallinity (in mass fractions of minerals) of HeatTreated Glasses with Hematite

\begin{tabular}{|l|c|c|c|c||}
\hline Glass & Cristobalite & Trevorite & Hematite & Sum \\
\hline CVS1-12 & & & 0.02 & 0.02 \\
\hline CVS2-30 & 0.02 & & 0.09 & 0.11 \\
\hline CVS2-79 & 0.20 & 0.05 & 0.01 & 0.26 \\
\hline
\end{tabular}


Table 3.19. Crystallinity (in mass fractions of minerals) of HeatTreated Glasses with zircon

\begin{tabular}{|l|c|c|c|c||}
\hline Glass & Baddeleyite & Cristobalite & Zircon & Sum \\
\hline CVS1-6 & 0.01 & & 0.09 & 0.10 \\
\hline CVS2-78 & & 0.22 & 0.03 & 0.25 \\
\hline CVS2-80 & & 0.15 & 0.09 & 0.24 \\
\hline CVS3-20 & & & 0.10 & 0.10 \\
\hline CVS3-21 & & & 0.10 & 0.10 \\
\hline
\end{tabular}

Table 3.20. Residual Glass Composition (in mass fractions of oxides): Glasses Precipitating Lithium-Silicate

\begin{tabular}{|l|c|c|c|c|c|c|c|c|c|c||}
\hline Glass & $\mathbf{A l}_{2} \mathbf{O}_{\mathbf{3}}$ & $\mathbf{B}_{2} \mathbf{O}_{\mathbf{3}}$ & $\mathbf{C a O}$ & $\mathbf{F e}_{2} \mathbf{O}_{3}$ & $\mathbf{L i}_{2} \mathbf{O}$ & $\mathbf{M g O}$ & $\mathbf{N a}_{2} \mathbf{O}$ & $\mathbf{S i O}_{2}$ & $\mathbf{Z r O}_{2}$ & Others \\
\hline CVS1-5 & 0.084 & 0.053 & 0.000 & 0.129 & 0.070 & 0.000 & 0.074 & 0.593 & 0.000 & 0.000 \\
\hline CVS1-18 & 0.000 & 0.059 & 0.024 & 0.024 & 0.073 & 0.094 & 0.140 & 0.476 & 0.006 & 0.106 \\
\hline CVS2-22 & 0.042 & 0.058 & 0.000 & 0.023 & 0.054 & 0.060 & 0.085 & 0.560 & 0.000 & 0.116 \\
\hline CVS2-39 & 0.029 & 0.056 & 0.000 & 0.082 & 0.044 & 0.079 & 0.151 & 0.470 & 0.078 & 0.011 \\
\hline CVS2-68 & 0.182 & 0.151 & 0.001 & 0.005 & 0.048 & 0.000 & 0.089 & 0.501 & 0.000 & 0.024 \\
\hline CVS2-70 & 0.204 & 0.159 & 0.001 & 0.009 & 0.040 & 0.001 & 0.091 & 0.471 & 0.001 & 0.022 \\
\hline CVS2-76 & 0.021 & 0.063 & 0.042 & 0.111 & 0.049 & 0.005 & 0.189 & 0.493 & 0.005 & 0.021 \\
\hline CVS2-81 & 0.022 & 0.066 & 0.005 & 0.078 & 0.062 & 0.044 & 0.191 & 0.454 & 0.005 & 0.072 \\
\hline CVS2-82 & 0.021 & 0.099 & 0.005 & 0.005 & 0.059 & 0.042 & 0.188 & 0.556 & 0.005 & 0.021 \\
\hline CVS2-84 & 0.021 & 0.063 & 0.005 & 0.111 & 0.056 & 0.005 & 0.189 & 0.444 & 0.084 & 0.021 \\
\hline CVS2-90 & 0.022 & 0.172 & 0.005 & 0.101 & 0.072 & 0.005 & 0.058 & 0.541 & 0.005 & 0.018 \\
\hline
\end{tabular}

Table 3.21. Residual Glass Composition (in mass fractions of oxides): Glasses Precipitating Cristobalite

\begin{tabular}{|l|c|c|c|c|c|c|c|c|c|c|}
\hline \hline Glass & $\mathbf{A l}_{\mathbf{2}} \mathbf{O}_{\mathbf{3}}$ & $\mathbf{B}_{2} \mathbf{O}_{\mathbf{3}}$ & $\mathbf{C a O}$ & $\mathbf{F e}_{2} \mathbf{O}_{\mathbf{3}}$ & $\mathbf{L i}_{2} \mathbf{O}$ & $\mathbf{M g O}$ & $\mathbf{N a}_{2} \mathbf{O}$ & $\mathbf{S i O}_{2}$ & $\mathbf{Z r O}_{2}$ & Others \\
\hline CVS1-9 & 0.000 & 0.213 & 0.000 & 0.100 & 0.011 & 0.087 & 0.058 & 0.446 & 0.000 & 0.084 \\
\hline CVS2-30 & 0.051 & 0.225 & 0.000 & 0.047 & 0.011 & 0.000 & 0.093 & 0.561 & 0.000 & 0.011 \\
\hline CVS2-77 & 0.020 & 0.061 & 0.005 & 0.005 & 0.071 & 0.005 & 0.184 & 0.444 & 0.112 & 0.092 \\
\hline CVS2-78 & 0.027 & 0.213 & 0.007 & 0.093 & 0.034 & 0.053 & 0.067 & 0.438 & 0.042 & 0.027 \\
\hline CVS2-79 & 0.027 & 0.216 & 0.007 & 0.082 & 0.016 & 0.007 & 0.068 & 0.470 & 0.007 & 0.100 \\
\hline CVS2-80 & 0.026 & 0.211 & 0.007 & 0.138 & 0.023 & 0.053 & 0.066 & 0.423 & 0.027 & 0.026 \\
\hline CVS2-90 & 0.022 & 0.172 & 0.005 & 0.101 & 0.072 & 0.005 & 0.058 & 0.541 & 0.005 & 0.018 \\
\hline CVS2-91 & 0.020 & 0.162 & 0.005 & 0.005 & 0.010 & 0.040 & 0.106 & 0.556 & 0.005 & 0.091 \\
\hline
\end{tabular}


Table 3.22. Residual Glass Composition (in mass fractions of oxides): Glasses Precipitating Hematite

\begin{tabular}{|l|c|c|c|c|c|c|c|c|c|c||}
\hline Glass & $\mathbf{A l}_{2} \mathbf{O}_{\mathbf{3}}$ & $\mathbf{B}_{2} \mathbf{O}_{\mathbf{3}}$ & $\mathbf{C a O}$ & $\mathbf{F e}_{2} \mathbf{O}_{3}$ & $\mathbf{L i}_{2} \mathbf{O}$ & $\mathbf{M g O}$ & $\mathbf{N a}_{2} \mathbf{O}$ & $\mathbf{S i O}_{2}$ & $\mathbf{Z r O}_{2}$ & Others \\
\hline CVS1-12 & 0.000 & 0.179 & 0.102 & 0.137 & 0.071 & 0.000 & 0.075 & 0.426 & 0.000 & 0.010 \\
\hline CVS2-30 & 0.051 & 0.225 & 0.000 & 0.047 & 0.011 & 0.000 & 0.093 & 0.561 & 0.000 & 0.011 \\
\hline CVS2-79 & 0.027 & 0.216 & 0.007 & 0.082 & 0.016 & 0.007 & 0.068 & 0.470 & 0.007 & 0.100 \\
\hline
\end{tabular}

Table 3.23. Residual Glass Composition (in mass fractions of oxides): Glasses Precipitating Zircon

\begin{tabular}{|l|l|l|l|l|l|l|l|l|l|l|}
\hline Glass & $\mathbf{A l}_{2} \mathbf{O}_{3}$ & $\mathbf{B}_{2} \mathbf{O}_{3}$ & $\mathbf{C a O}$ & $\mathbf{F e}_{2} \mathbf{O}_{3}$ & $\mathbf{L i}_{2} \mathbf{O}$ & $\mathbf{M g O}$ & $\mathbf{N a}_{2} \mathbf{O}$ & $\mathbf{S i O}_{2}$ & $\mathbf{Z r O}_{2}$ & Others \\
\hline CVS1-6 & 0.000 & 0.222 & 0.000 & 0.022 & 0.078 & 0.000 & 0.056 & 0.449 & 0.062 & 0.111 \\
\hline CVS2-78 & 0.027 & 0.213 & 0.007 & 0.093 & 0.034 & 0.053 & 0.067 & 0.438 & 0.042 & 0.027 \\
\hline CVS2-80 & 0.026 & 0.211 & 0.007 & 0.138 & 0.023 & 0.053 & 0.066 & 0.423 & 0.027 & 0.026 \\
\hline CVS3-20 & 0.047 & 0.000 & 0.012 & 0.066 & 0.028 & 0.000 & 0.123 & 0.476 & 0.111 & 0.135 \\
\hline CVS3-21 & 0.050 & 0.000 & 0.013 & 0.069 & 0.029 & 0.000 & 0.129 & 0.501 & 0.066 & 0.142 \\
\hline
\end{tabular}

Table 3.24. Measured $r_{B}$ and $r_{N a}$ (in $\mathrm{g} / \mathrm{m}^{2}$ ) from Quenched and Heat-Treated Glasses with Lithium-Silicate

\begin{tabular}{|l|r|r|r|r||}
\hline Glass & \multicolumn{1}{|c|}{$\mathbf{Q}, \mathbf{M}$} & $\mathbf{Q}, \mathbf{M}$ & $\mathbf{H}, \mathbf{M}$ & $\mathbf{H}, \mathbf{M}$ \\
\hline & \multicolumn{1}{c|}{$\boldsymbol{r}_{\boldsymbol{B}}$} & \multicolumn{1}{|c|}{$\boldsymbol{r}_{\boldsymbol{N} \boldsymbol{a}}$} & \multicolumn{1}{c|}{$\boldsymbol{r}_{\boldsymbol{h} \boldsymbol{B}}$} & $\boldsymbol{r}_{\boldsymbol{h N a}}$ \\
\hline CVS1-5 & 0.36 & 0.19 & 0.89 & 0.50 \\
\hline CVS1-18 & 11.24 & 8.04 & 22.19 & 12.22 \\
\hline CVS2-22 & 9.65 & 6.75 & 5.93 & 3.50 \\
\hline CVS2-39 & 12.60 & 7.67 & 15.39 & 8.32 \\
\hline CVS2-68 & 0.31 & 0.13 & 11.31 & 2.94 \\
\hline CVS2-70 & 0.32 & 0.13 & 13.15 & 3.64 \\
\hline CVS2-76 & 14.87 & 12.54 & 13.13 & 9.55 \\
\hline CVS2-81 & 16.62 & 12.83 & 20.35 & 15.14 \\
\hline CVS2-82 & 44.01 & 35.38 & - & - \\
\hline CVS2-84 & 12.46 & 8.24 & 20.36 & 12.73 \\
\hline CVS2-90 & 5.58 & 3.49 & 6.13 & 3.92 \\
\hline
\end{tabular}


Table 3.25. Measured $r_{B}$ and $r_{N a}$ (in $\mathrm{g} / \mathrm{m}^{2}$ ) from Quenched and Heat-Treated Glasses with Cristobalite

\begin{tabular}{|l|c|c|c|c|}
\hline Glass & $\mathbf{Q}, \mathbf{M}$ & $\mathbf{Q}, \mathbf{M}$ & $\mathbf{H}, \mathbf{M}$ & $\mathbf{H}, \mathbf{M}$ \\
\hline & $\boldsymbol{r}_{\boldsymbol{B}}$ & $\boldsymbol{r}_{\boldsymbol{N} \boldsymbol{a}}$ & $\boldsymbol{r}_{\boldsymbol{h} \boldsymbol{B}}$ & $\boldsymbol{r}_{\boldsymbol{h N a}}$ \\
\hline CVS1-9 & 1.235 & 0.81 & 4.18 & 1.66 \\
\hline CVS2-30 & 6.51 & 2.55 & 18.005 & 8.685 \\
\hline CVS2-77 & 9.515 & 6.45 & 8.04 & 4.16 \\
\hline CVS2-78 & 0.935 & 0.59 & 19.09 & 14.83 \\
\hline CVS2-79 & 0.74 & 0.66 & 43.105 & 35.715 \\
\hline CVS2-80 & 0.76 & 0.6 & 25.775 & 21.16 \\
\hline CVS2-90 & 5.58 & 3.485 & 6.13 & 3.92 \\
\hline CVS2-91 & 8.64 & 6.74 & 8.24 & 6.42 \\
\hline \hline
\end{tabular}

Table 3.26. Measured $r_{B}$ and $r_{N a}\left(\right.$ in $\left.\mathrm{g} / \mathrm{m}^{2}\right)$ from Quenched and Heat-Treated Glasses with Hematite

\begin{tabular}{|l|c|c|c|c||}
\hline Glass & $\mathbf{Q}, \mathbf{M}$ & $\mathbf{Q}, \mathbf{M}$ & $\mathbf{H}, \mathbf{M}$ & $\mathbf{H}, \mathbf{M}$ \\
\hline & $\boldsymbol{r}_{\boldsymbol{B}}$ & $\boldsymbol{r}_{\boldsymbol{N} \boldsymbol{a}}$ & $\boldsymbol{r}_{\boldsymbol{h} \boldsymbol{B}}$ & $\boldsymbol{r}_{\boldsymbol{h N a} \boldsymbol{a}}$ \\
\hline CVS1-12 & 4.66 & 4.38 & 2.99 & 4.08 \\
\hline CVS2-30 & 6.51 & 2.55 & 18.01 & 8.69 \\
\hline CVS2-79 & 0.74 & 0.66 & 43.11 & 35.72 \\
\hline
\end{tabular}

Table 3.27. Measured $r_{B}$ and $r_{N a}\left(\right.$ in $\left.\mathrm{g} / \mathrm{m}^{2}\right)$ from Quenched and Heat-Treated Glasses with zircon

\begin{tabular}{|l|c|c|r|r||}
\hline & \multicolumn{2}{|c|}{ Quenched } & \multicolumn{2}{c|}{ CCC } \\
\hline Glass & $\mathbf{Q}, \mathbf{M}$ & $\mathbf{Q}, \mathbf{M}$ & $\mathbf{H}, \mathbf{M}$ & $\mathbf{H}, \mathbf{M}$ \\
\hline & $\boldsymbol{r}_{\boldsymbol{B}}$ & $\boldsymbol{r}_{\boldsymbol{N} \boldsymbol{a}}$ & \multicolumn{1}{c|}{$\boldsymbol{r}_{\boldsymbol{h} \boldsymbol{B}}$} & $\boldsymbol{r}_{\boldsymbol{h N a}}$ \\
\hline CVS1-6 & 6.11 & 3.05 & 5.04 & 2.80 \\
\hline CVS2-78 & 0.94 & 0.59 & 19.09 & 14.83 \\
\hline CVS2-80 & 0.76 & 0.60 & 25.78 & 21.16 \\
\hline CVS3-20 & 0.18 & 0.30 & 0.80 & 0.36 \\
\hline CVS3-21 & 0.20 & 0.25 & 0.53 & 0.32 \\
\hline
\end{tabular}


Table 3.28. Calculated $r_{B}$ and $r_{N a}$ (in $\mathrm{g} / \mathrm{m}^{2}$ ) from Quenched and Heat-Treated Glasses with Lithium Silicate

\begin{tabular}{||l|r|r|c|c|c|c||}
\hline Glass & $\mathbf{Q}, \mathbf{C}$ & $\mathbf{Q}, \mathbf{C}$ & $\mathbf{H}, \mathbf{Q C}, \mathbf{R C}$ & $\mathbf{H}, \mathbf{Q C}, \mathbf{R C}$ & $\mathbf{H}, \mathbf{Q M}, \mathbf{R C}$ & $\mathbf{H}, \mathbf{Q M}, \mathbf{R C}$ \\
\hline & \multicolumn{1}{|c|}{$\boldsymbol{r}_{\boldsymbol{B}}$} & $\boldsymbol{r}_{\boldsymbol{N} \boldsymbol{a}}$ & \multicolumn{1}{c|}{$\boldsymbol{r}_{\boldsymbol{r} \boldsymbol{B}}$} & $\boldsymbol{r}_{\boldsymbol{r} \boldsymbol{N} \boldsymbol{a}}$ & $\boldsymbol{r}_{\boldsymbol{r} \boldsymbol{B}}$ & $\boldsymbol{r}_{\boldsymbol{r} \boldsymbol{N} \boldsymbol{a}}$ \\
\hline & \multicolumn{1}{|c|}{} & \multicolumn{2}{c|}{ with $\boldsymbol{r}_{\boldsymbol{j}}$ calculated } & with $\boldsymbol{r}_{\boldsymbol{j}}$ measured \\
\hline \hline CVS1-5 & 0.21 & 0.13 & 0.20 & 0.13 & 0.35 & 0.19 \\
\hline CVS1-18 & 5.60 & 4.60 & 31.29 & 29.25 & 71.06 & 56.36 \\
\hline CVS2-22 & 2.48 & 1.80 & 1.71 & 1.26 & 8.28 & 5.89 \\
\hline CVS2-39 & 5.64 & 4.45 & 3.84 & 3.31 & 9.37 & 6.05 \\
\hline CVS2-68 & 0.17 & 0.10 & 0.09 & 0.06 & 0.18 & 0.08 \\
\hline CVS2-70 & 0.13 & 0.08 & 0.06 & 0.04 & 0.16 & 0.07 \\
\hline CVS2-76 & 7.11 & 7.55 & 6.18 & 7.03 & 13.43 & 11.98 \\
\hline CVS2-81 & 19.34 & 15.46 & 23.79 & 20.60 & 20.13 & 16.77 \\
\hline CVS2-82 & 26.82 & 22.55 & 25.37 & 22.29 & 42.50 & 35.63 \\
\hline CVS2-84 & 6.23 & 4.81 & 5.38 & 4.37 & 11.16 & 7.70 \\
\hline CVS2-90 & 3.21 & 1.62 & 4.31 & 2.12 & 7.80 & 4.83 \\
\hline
\end{tabular}

Table 3.29. Calculated $r_{B}$ and $r_{N a}$ (in $\mathrm{g} / \mathrm{m}^{2}$ ) from Quenched and Heat-Treated Glasses with Cristobalite

\begin{tabular}{|l|c|c|c|c|c|c||}
\hline Glass & $\mathbf{Q}, \mathbf{C}$ & $\mathbf{Q}, \mathbf{C}$ & $\mathbf{H}, \mathbf{Q C}, \mathbf{R C}$ & $\mathbf{H}, \mathbf{Q C}, \mathbf{R C}$ & $\mathbf{H}, \mathbf{Q M}, \mathbf{R C}$ & $\mathbf{H}, \mathbf{Q M}, \mathbf{R C}$ \\
\hline & $\boldsymbol{r}_{\boldsymbol{B}}$ & $\boldsymbol{r}_{\boldsymbol{N} \boldsymbol{a}}$ & \multicolumn{2}{|c|}{$\boldsymbol{r}_{\boldsymbol{r} \boldsymbol{B}}$} & $\boldsymbol{r}_{\boldsymbol{r} \boldsymbol{N} \boldsymbol{a}}$ & \multicolumn{2}{c|}{$\boldsymbol{r}_{\boldsymbol{r} \boldsymbol{B}}$} & $\boldsymbol{r}_{\boldsymbol{r} \boldsymbol{a} \boldsymbol{a}}$ \\
\hline & \multicolumn{7}{|c|}{ with $\boldsymbol{r}_{\boldsymbol{j}}$ calculated } & \multicolumn{2}{c|}{ with $\boldsymbol{r}_{\boldsymbol{j}}$ measured } \\
\hline CVS1-9 & 8.61 & 4.63 & 12.79 & 6.98 & 1.55 & 1.05 \\
\hline CVS2-30 & 1.30 & 0.73 & 2.05 & 1.17 & 12.50 & 4.79 \\
\hline CVS2-77 & 6.33 & 4.92 & 7.18 & 5.56 & 10.88 & 7.33 \\
\hline CVS2-78 & 1.10 & 0.66 & 5.56 & 2.93 & 4.49 & 2.55 \\
\hline CVS2-79 & 0.85 & 0.49 & 3.10 & 1.62 & 2.58 & 2.43 \\
\hline CVS2-80 & 0.79 & 0.46 & 4.49 & 2.40 & 4.26 & 3.39 \\
\hline CVS2-90 & 3.21 & 1.62 & 4.31 & 2.12 & 7.80 & 4.83 \\
\hline CVS2-91 & 4.08 & 2.95 & 4.32 & 3.12 & 9.22 & 7.18 \\
\hline \hline
\end{tabular}


Table 3.30. Calculated $r_{B}$ and $r_{N a}$ (in $\mathrm{g} / \mathrm{m}^{2}$ ) from Quenched and Heat-Treated Glasses with Hematite

\begin{tabular}{|l|c|c|c|c|c|c|}
\hline Glass & $\mathbf{Q}, \mathbf{C}$ & $\mathbf{Q}, \mathbf{C}$ & $\mathbf{H}, \mathbf{Q C}, \mathbf{R C}$ & $\mathbf{H}, \mathbf{Q C}, \mathbf{R C}$ & $\mathrm{H}, \mathbf{Q M}, \mathbf{R C}$ & H, QM, RC \\
\hline & $\boldsymbol{r}_{\boldsymbol{B}}$ & $\boldsymbol{r}_{\boldsymbol{N} \boldsymbol{a}}$ & \multicolumn{2}{c|}{$\boldsymbol{r}_{\boldsymbol{r} \boldsymbol{B}}$} & $\boldsymbol{r}_{\boldsymbol{r} \boldsymbol{a}}$ & \multicolumn{2}{c|}{$\boldsymbol{r}_{\boldsymbol{r} \boldsymbol{B}}$} & $\boldsymbol{r}_{\boldsymbol{r} \boldsymbol{a} \boldsymbol{a}}$ \\
\hline & \multicolumn{2}{|c|}{} & \multicolumn{2}{c|}{ with $\boldsymbol{r}_{\boldsymbol{j}}$ calculated } & \multicolumn{2}{c|}{ with $\boldsymbol{r}_{\boldsymbol{j}}$ measured } \\
\hline CVS1-12 & 6.24 & 5.78 & 6.73 & 6.32 & 5.01 & 4.77 \\
\hline CVS2-30 & 1.30 & 0.73 & 2.05 & 1.17 & 12.50 & 4.79 \\
\hline CVS2-79 & 0.85 & 0.49 & 3.10 & 1.62 & 2.58 & 2.43 \\
\hline \hline
\end{tabular}

Table 3.31. Calculated $r_{B}$ and $r_{N a}$ (in $\mathrm{g} / \mathrm{m}^{2}$ ) from Quenched and Heat-Treated Glasses with Zircon

\begin{tabular}{|l|c|c|c|c|c|c||}
\hline Glass & Q, C & Q, C & H, QC, RC & H, QC, RC & H, QM, RC & H, QM, RC \\
\hline & $\boldsymbol{r}_{\boldsymbol{B}}$ & $\boldsymbol{r}_{\boldsymbol{N} \boldsymbol{a}}$ & \multicolumn{2}{|c|}{$\boldsymbol{r}_{\boldsymbol{r} \boldsymbol{B}}$} & $\boldsymbol{r}_{\boldsymbol{r} \boldsymbol{a}}$ & \multicolumn{2}{c|}{$\boldsymbol{r}_{\boldsymbol{r} \boldsymbol{B}}$} & $\boldsymbol{r}_{\boldsymbol{r} \boldsymbol{a} \boldsymbol{a}}$ \\
\hline & \multicolumn{7}{|c|}{} & \multicolumn{2}{c|}{ with $\boldsymbol{r}_{\boldsymbol{j}}$ calculated } & \multicolumn{2}{c||}{ with $\boldsymbol{r}_{\boldsymbol{j}}$ measured } \\
\hline \hline CVS1-6 & 5.18 & 2.07 & 15.70 & 6.06 & 18.85 & 9.28 \\
\hline CVS2-78 & 1.10 & 0.66 & 5.56 & 2.93 & 4.49 & 2.55 \\
\hline CVS2-80 & 0.79 & 0.46 & 4.49 & 2.40 & 4.26 & 3.39 \\
\hline CVS3-20 & 0.08 & 0.07 & 0.15 & 0.13 & 0.37 & 0.69 \\
\hline CVS3-21 & 0.12 & 0.10 & 0.22 & 0.21 & 0.41 & 0.56 \\
\hline \hline
\end{tabular}

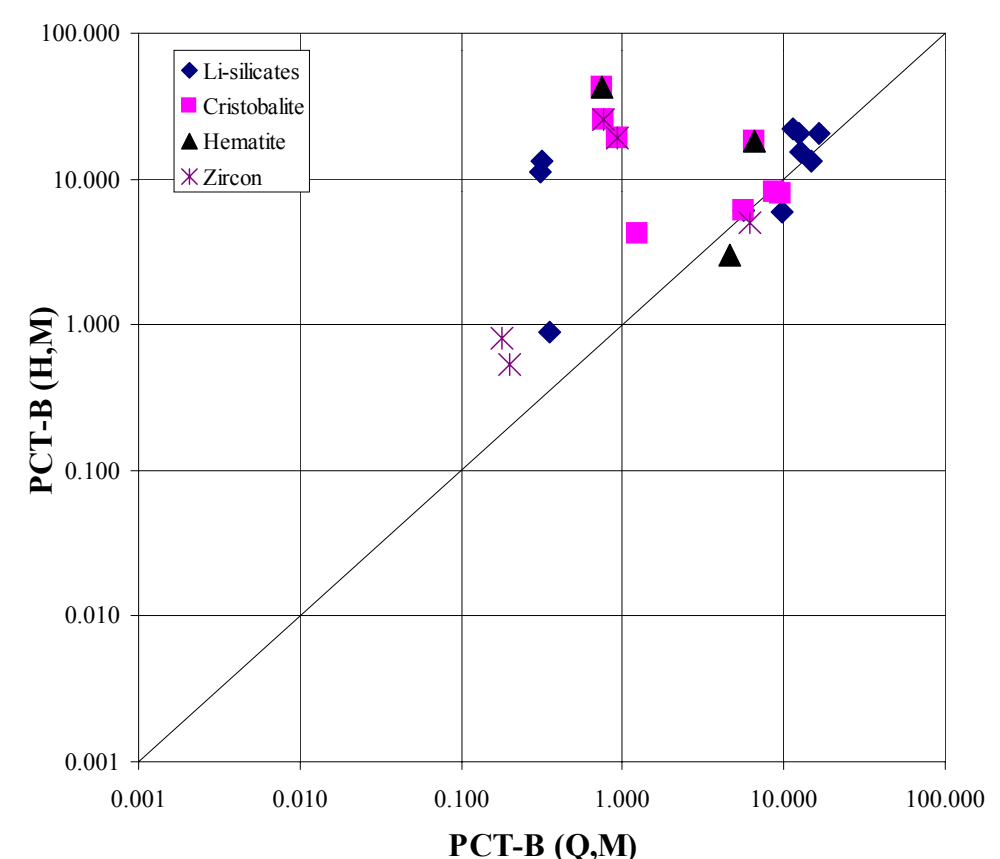

Figure 3-21. Glasses Precipitating other Crystalline Phases: $r_{h B}$ vs. $r_{B}$ 


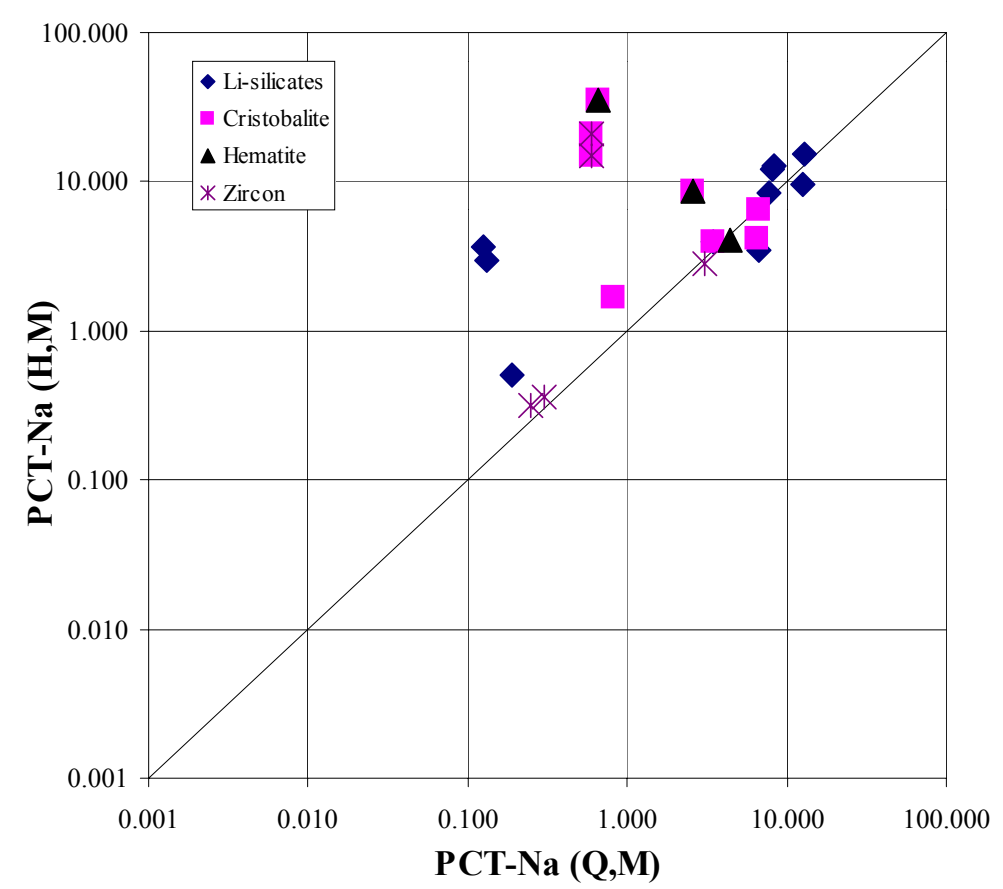

Figure 3-22. Glasses Precipitating other Crystalline Phases: $\boldsymbol{r}_{h N a}$ vs. $\boldsymbol{r}_{N a}$

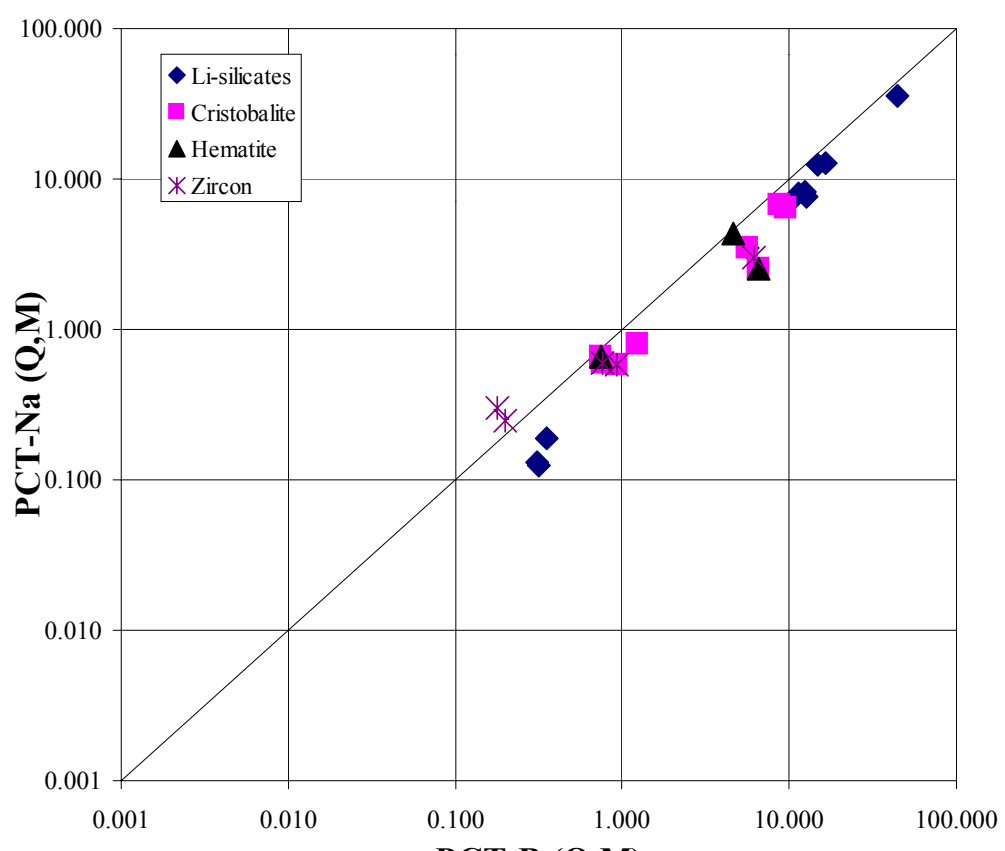

PCT-B (Q,M)

Figure 3-23. Glasses Precipitating other Crystalline Phases: $r_{N a}$ vs. $r_{B}$ 


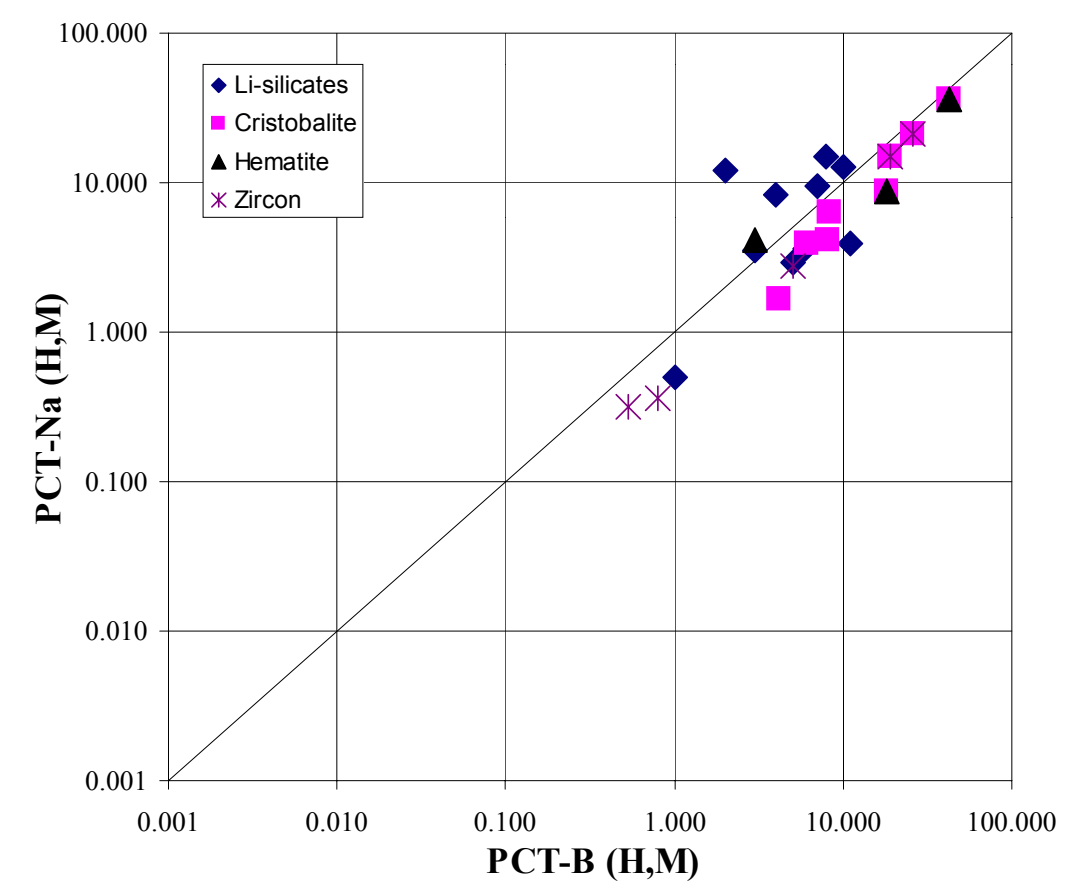

Figure 3-24. Glasses Precipitating other Crystalline Phases: $r_{h N a}$ vs. $r_{h B}$

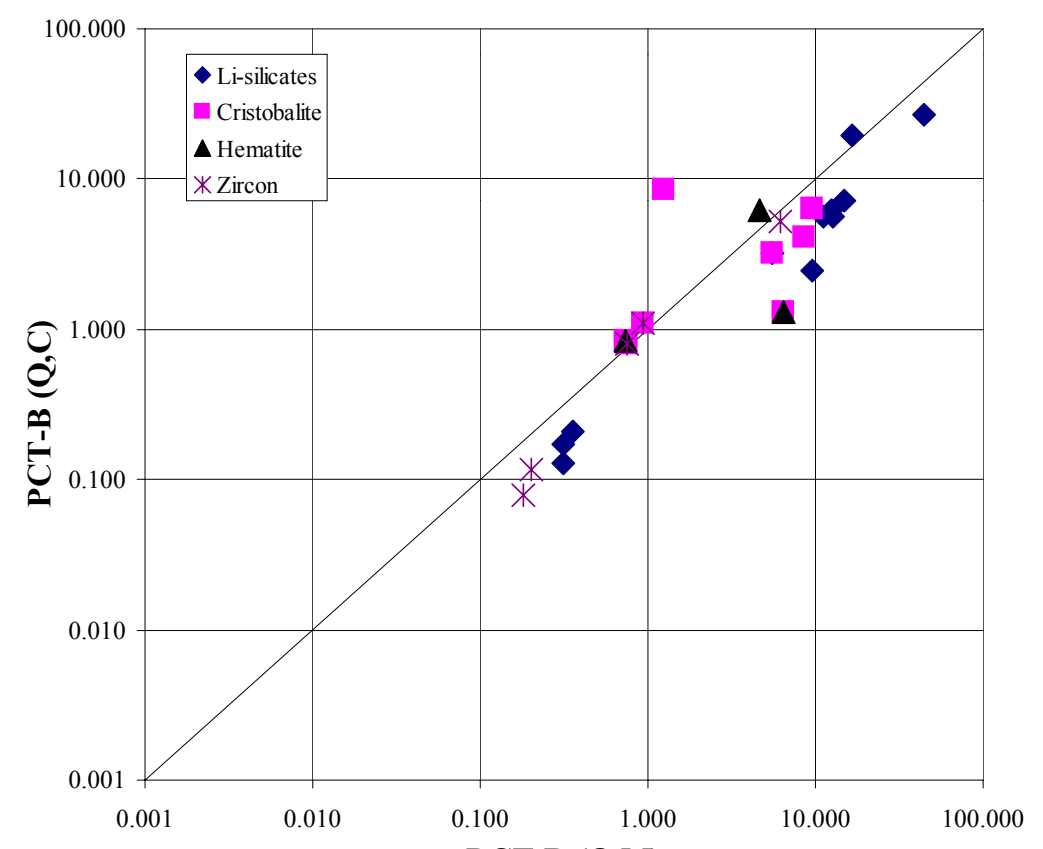

PCT-B (Q,M)

Figure 3-25. Glasses Precipitating other Crystalline Phases: $r_{B}$, Calculated vs. Measured 


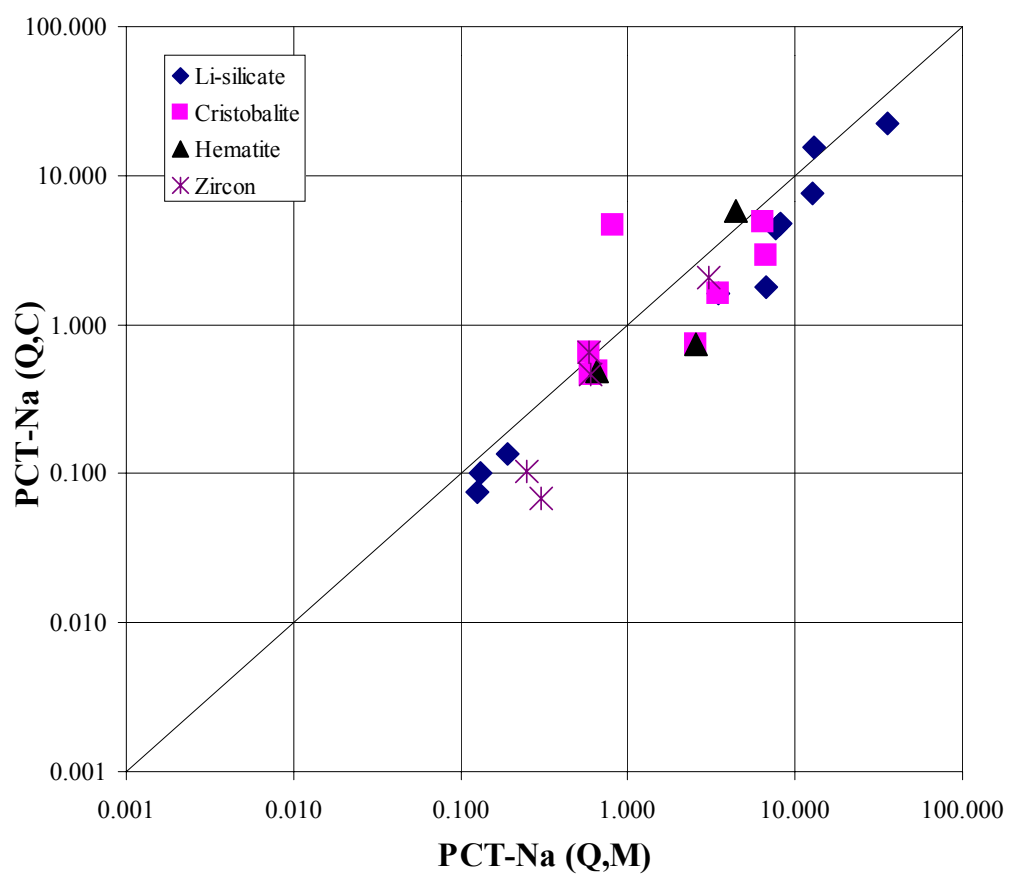

Figure 3-26. Glasses Precipitating other Crystalline Phases: $r_{\mathrm{Na}}$ Calculated vs. Measured

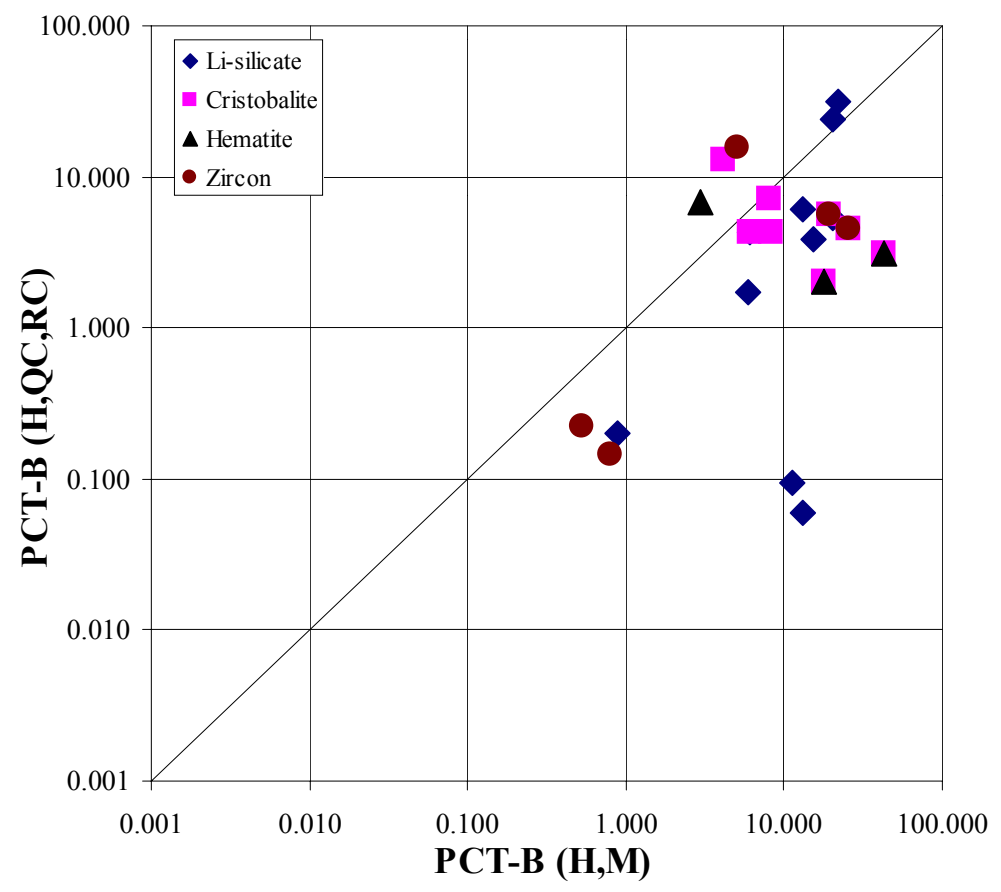

Figure 3-27. Glasses Precipitating other Crystalline Phases: $r_{r B}$ 
(calculated) vs. $r_{h B}$ (measured) 


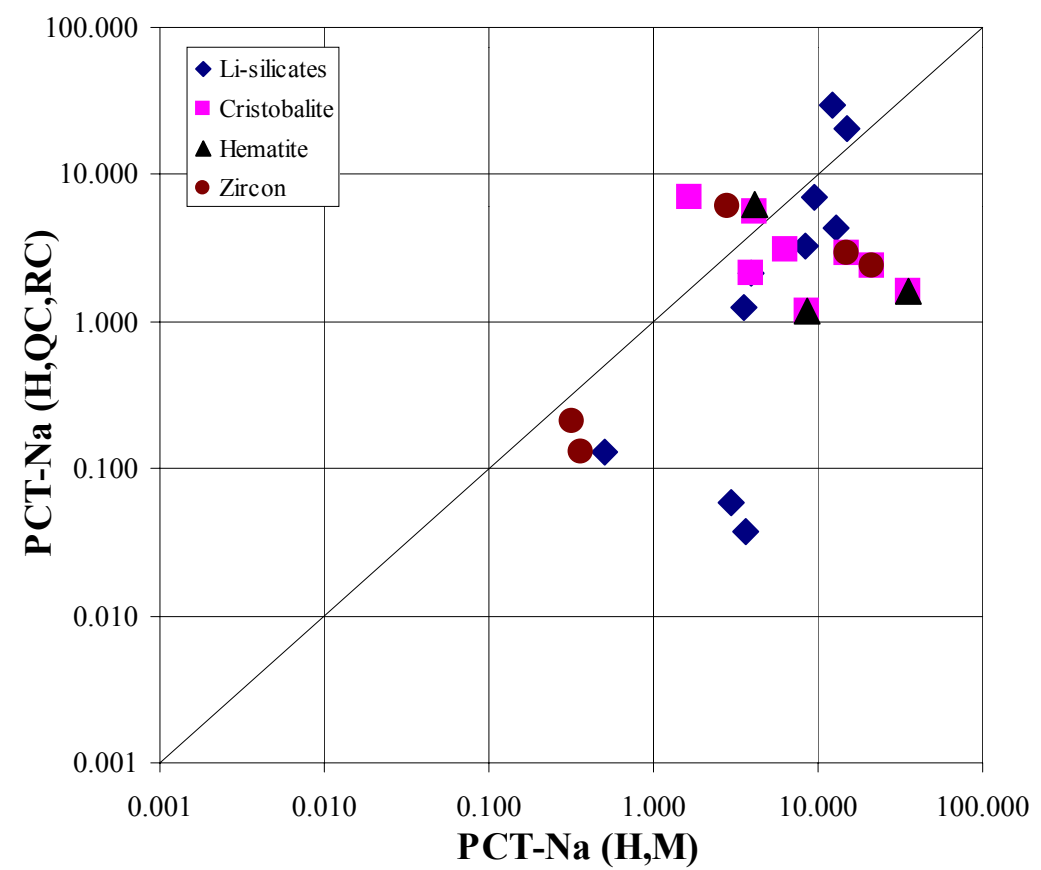

Figure 3-28. Glasses Precipitating other Crystalline Phases: $r_{r N a}$ (calculated) vs. $r_{h N a}$ (measured)

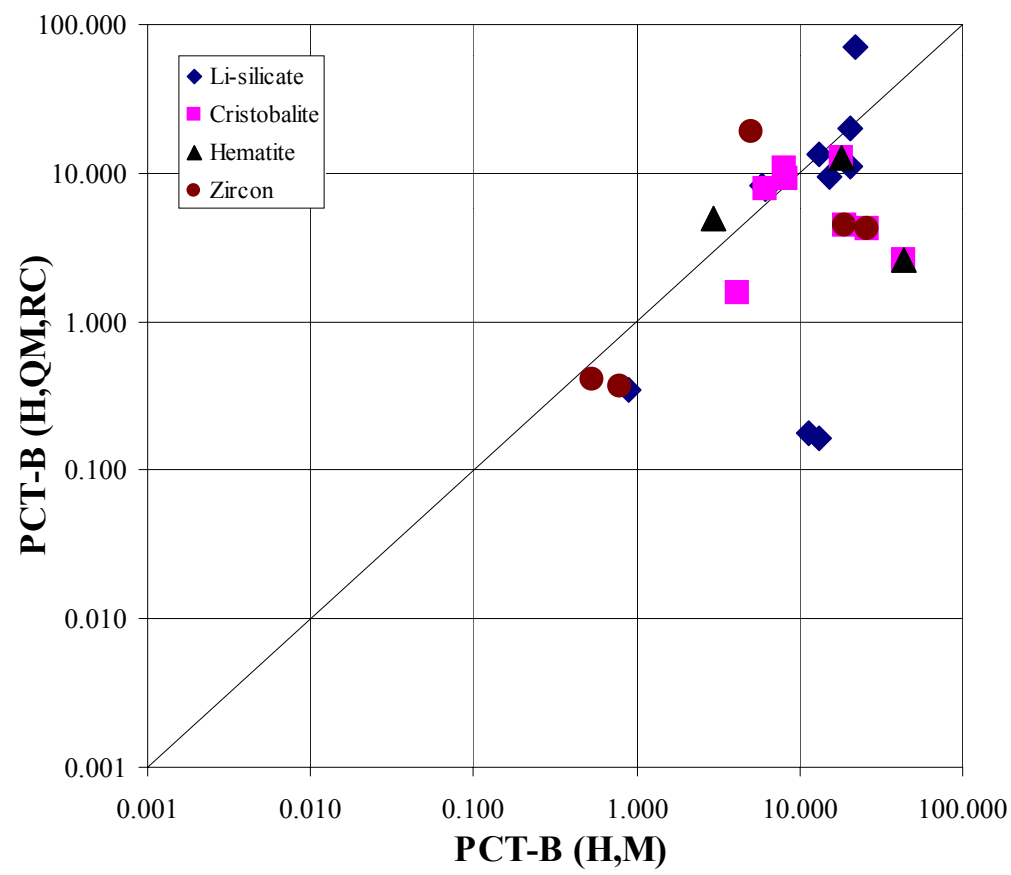

Figure 3-29. Glasses Precipitating other Crystalline Phases: $r_{x B}$ 
(calculated using measured $r_{B}$ ) vs. $r_{h B}$ (measured) 


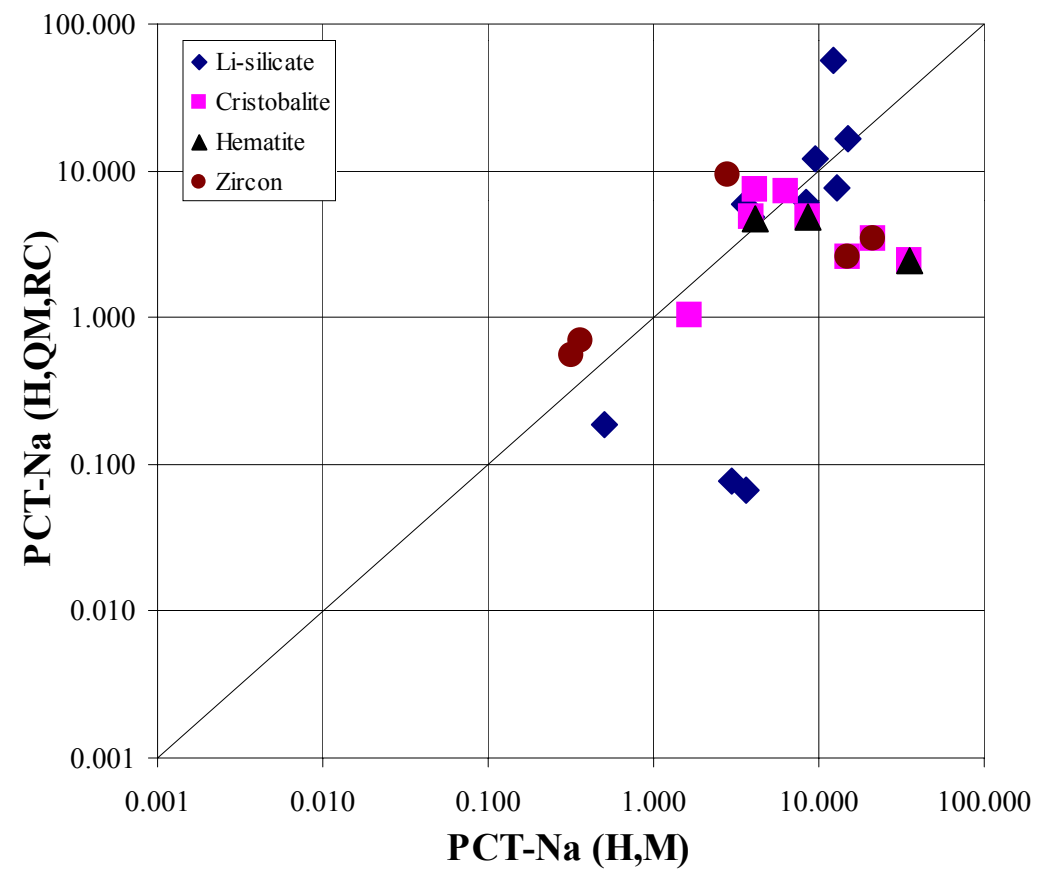

Figure 3-30. Glasses Precipitating other Crystalline Phases: $r_{r N a}$ (calculated using measured $r_{N a}$ ) vs. $r_{h N a}$ (measured) 


\section{0 Conclusions and Recommendations}

Previous studies showed that precipitation of cristobalite and certain aluminosilicates could decrease chemical durability by several orders of magnitude, whereas severe crystallization of other phases has little or even positive impact on glass durability. This behavior can be attributed to changes of the residual glass composition associated with crystallization. This effect can be predicted by computing residual glass composition based on the mass balance of components and estimating PCT releases from the residual glass from first-order models that have been developed for crystal-free glasses. Glasses of Hanford and Savannah River composition regions were sorted out into several groups according to the crystalline phases that precipitated or potentially precipitated upon heat treatment. These phases are clinopyroxene, NP, lithium silicate, cristobalite, hematite, and zircon.

This report provides

1. a summary of compositions of 212 glasses from previous studies conducted at PNNL and SRTC that precipitated crystalline phases upon CCC or IHT, sorted out by major mineral phases

2. crystallinity fractions for individual phases measured by semiquantitative XRD

3. residual glass compositions calculated using mass balances of components

4. measured $r_{B}$ and $r_{N a}$ from quenched and heat-treated glasses

5. calculated $r_{B}$ and $r_{N a}$ from quenched glasses

6. calculated $r_{B}$ and $r_{N a}$ from heat-treated glasses using two methods of calculation.

Reasonable agreement exists between calculated and measured values of PCT B and Na- releases, but numerous outliers were identified in each group of glasses. The likely cause of the discrepancies is that the composition of residual glass is outside the region for which the models were established, but other causes, such as the low accuracy of semiquantitative XRD and secondary effects are also considered.

Recommendations:

1. use quantitative XRD to obtain more accurate values for the crystallinity of the durabilityaffecting phases

2. use SEM/EDS to determine the composition of these crystalline phases

3. generate PCT data for simulated residual glass compositions and extend PCT models to cover the residual glass-composition region

4. develop a method for predicting compositions and concentrations of the durability-affecting phases in CCC glass

5. extend glass formulation methodology to include $r_{B}$ and $r_{N a}$ from glasses subjected to CCC. 


\subsection{References}

American Society for Testing and Materials (ASTM). 1998. "Standard Test Methods for Determining Chemical Durability of Nuclear, Hazardous, and Mixed Waste Glasses: The Product Consistency Test (PCT)," C 1285-97 in 1998 Annual Book of ASTM Standards Vol. 12.01, ASTM, West Conshohocken, Pennsylvania.

Bailey, A. W., and P. Hrma. 1995. "Waste Loading Maximization for Vitrified Hanford HLW Blend," Ceram. Trans. 61, 549-556.

Casler, D. G., and P. Hrma. 1999. "Nonisothermal Kinetics of Spinel Crystallization in a HLW Glass," Mat. Res. Soc. Proc. 556, 255-262.

Cicero, C.A., S. L. Mara, and M. K. Andrews. 1993. Phase Stability Determinations of DWPF Waste Glasses (U), WSRC-TR-93-227, Westinghouse Savannah River Company, Aiken, South Carolina.

Edwards, R.E.. 1987. SGM Run 8 - Canister and Glass Temperature During Filling and Cooldown, DPST-87-801, Savannah River Laboratory, Aiken, South Carolina.

Hrma, P., G. F. Piepel, M. J. Schweiger, D. E. Smith, D-S. Kim, P. E. Redgate, J. D. Vienna, C. A. LoPresti, D. B. Simpson, D. K. Peeler, and M. H. Langowski. 1994. Property/Composition Relationships for Hanford High-Level Waste Glasses Melting at $1150^{\circ} \mathrm{C}$, PNL-10359, Vol. 1 and 2, Pacific Northwest Laboratory, Richland, Washington.

Hrma, P., and A. W. Bailey. 1995. "High Level Waste at Hanford: Potential for Waste Loading Maximization," Proc. 1995 Int. Conf. Nucl. Waste Manag. and Environ. Remediation (ICEM'95), Vol. 1, pp. 447-451.

Hrma, P., J. D. Vienna, J. V. Crum, G. F. Piepel, and M. Mika. 2000. "Liquidus Temperature of HighLevel Waste Borosilicate Glasses with Spinel Primary Phase," Mat. Res. Soc. Proc. 608, 671-676.

Hrma, P., G. F. Piepel, J. D. Vienna, P. E. Redgate, M. J. Schweiger, and D. E. Smith. 1995. "Prediction of Nuclear Waste Glass Dissolution as a Function of Composition," Ceram. Trans. 61, 497-504.

Jantzen, C.M. (1992), in D.E. Clark and K. Ziotos, Corrosion of Glass, Ceramics and Ceramic Superconductors, Noyes, Park Ridge, New Jersey.

Jantzen, C. M., N. E. Bibler, D. C. Beam, C. L. Crawford, and M. A. Pickett. 1993. Characterization of the Defense Waste Processing Facility (DWPF) Environmental Assessment (EA) Glass Standard Reference Material, WSRC-TR-92-346, Rev. 1, Westinghouse Savannah River Company, Aiken, South Carolina. 
Kim, D-S., D. K. Peeler, and P. Hrma. 1995. "Effects of Crystallization on the Chemical Durability of Nuclear Waste Glasses,” Ceram. Trans. 61, 177-185.

Lee, L. 1989. Thermal Analysis of DWPF Canister During Pouring and Cooldown, DPST-89-269- $T_{L}$, Savannah River Laboratory, Aiken, South Carolina.

Li, H., J. D. Vienna, P. Hrma, D. E. Smith, and M. J. Schweiger. 1997. "Nepheline Precipitation in HighLevel Waste Glasses - Compositional Effects and Impact on the Waste Form Acceptability," Mat. Res. Soc. Proc. 465, 261-268.

Marra, S. L., and C. M. Jantzen. 1993. Characterization of projected DWPF glasses heat treated to simulate canister centerline cooling, WSRC-TR-92-142, Rev. 1, Westinghouse Savannah River Company, Aiken, South Carolina.

Menkhaus, T. J., P. Hrma, and H. Li. 2000. "Kinetics of Nepheline Crystallization from High-level Waste Glass," Ceram. Trans. 107, 479-483.

Plaisted, T. J., F. Mo, B. Wilson, and P. Hrma. 2001. Surface Crystallization of Spinel and Acmite in High-Level Waste Glass," Ceram. Trans., in press.

Vienna, J.D., P. Hrma, M. J. Schweiger, M. H. Langowski, P. E. Redgate, D-S. Kim, G. F. Piepel, D. E. Smith, C. Y. Chang, D. E. Rinehart, S. E. Palmer, and H. Li. 1996. Effect of Composition and Temperature on the Properties of High-Level Waste (HLW) Glass Melting above $1200^{\circ} \mathrm{C}$, PNNL-10987, UC-810, Pacific Northwest Laboratory, Richland, Washington. 


\section{Addendum}

\section{Contents}

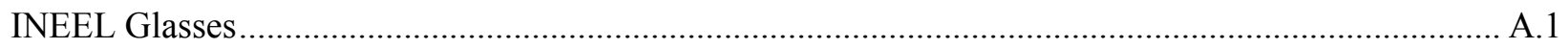

Renormalized Releases from Heat-Treated Glasses ................................................................... A.18

\section{Figures}

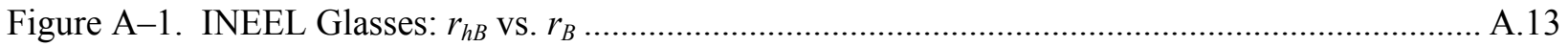

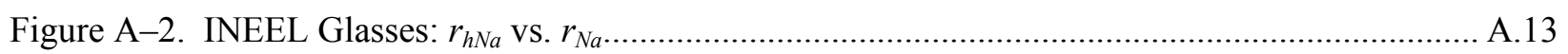

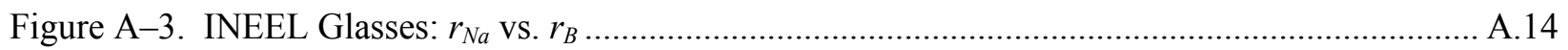

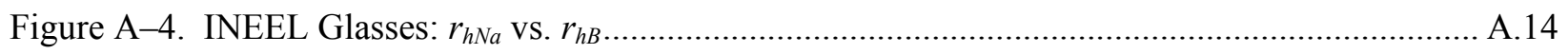

Figure A-5. INEEL Glasses: $r_{B}$, Calculated vs. Measured ............................................................ A.15

Figure A-6. INEEL Glasses: $r_{N a}$, Calculated vs. Measured............................................................. A.15

Figure A-7. INEEL Glasses: $r_{r B}$ (calculated) vs. $r_{h B}$ (measured) ...................................................... A.16

Figure A-8. INEEL Glasses: $r_{r N a}$ (calculated) vs. $r_{h N a}$ (measured) .................................................. A.16

Figure A-9. INEEL Glasses: $r_{r B}$ (calculated using measured $r_{B}$ ) vs. $r_{h B}$ (measured) ......................... A.17

Figure A-10. INEEL Glasses: $r_{r N a}$ (calculated using measured $r_{N a}$ ) vs. $r_{h N a}$ (measured) .................... A.17

Figure A-11. Mass Fraction of $\mathrm{B}_{2} \mathrm{O}_{3}$ in Glasses Precipitating Clinopyroxene, $g_{r B}$ vs. $g_{B} \ldots \ldots \ldots \ldots \ldots \ldots . . . . . .20$

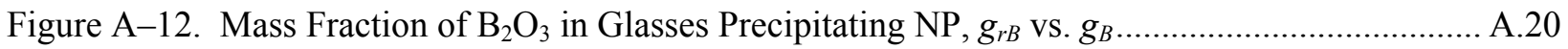

Figure A-13. Mass Fraction of $\mathrm{B}_{2} \mathrm{O}_{3}$ in Glasses Precipitating Phases other than Clinopyroxene

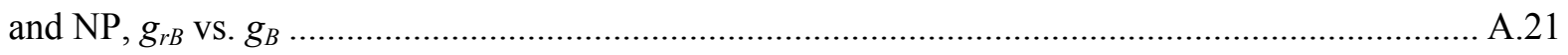

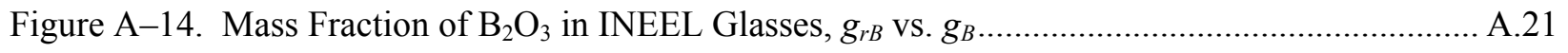

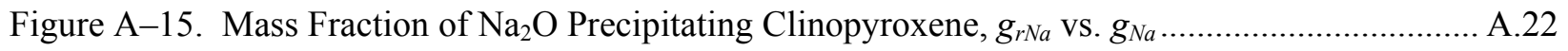

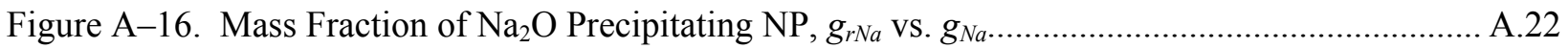

Figure A-17. Mass Fraction of $\mathrm{Na}_{2} \mathrm{O}$ in Glasses Precipitating Phases other than Clinopyroxene

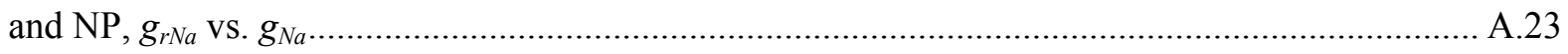

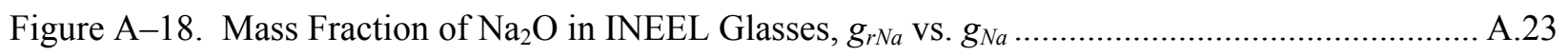

Figure A-19. Glasses Precipitating Clinopyroxene: $r_{h B}$, Residual vs. Original.................................. A.28 
Figure A-20. Glasses Precipitating Clinopyroxene: $r_{h N a}$, Residual vs. Original ............................... A.28

Figure A-21. Glasses Precipitating NP: $r_{h B}$, Residual vs. Original .................................................. A.29

Figure A-22. Glasses Precipitating NP: $r_{h N a}$, Residual vs. Original ................................................. A.29

Figure A-23. INEEL Glasses: $r_{h B}$, Residual vs. Original .............................................................. A.30

Figure A-24. INEEL Glasses: $r_{h N a}$, Residual vs. Original ............................................................... A.30

\section{Tables}

Table A-1. Component Coefficients for $r_{B}$ and $r_{N a}\left(\ln \text { values for } r_{i} \text { in } \mathrm{g} / \mathrm{m}^{2}\right)^{(1)}$ and Waste Glass

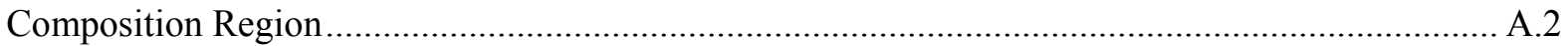

Table A-2. Composition of INEEL Glasses (in mass fractions of oxides)......................................... A.3

Table A-3. Minerals and their Effects on $r_{B}$ and $r_{N a}\left(\ln\right.$ values for $r_{i}$ in $\left.\mathrm{g} / \mathrm{m}^{2}\right)$ from INEEL Glasses ...... A.4

Table A-4. Crystallinity (in mass fractions of minerals) of Heat-Treated INEEL Glasses .................... A.5

Table A-5. Compositions of INEEL Residual Glasses (in mass fractions of oxides) ........................... A.7

Table A-6. Measured $r_{B}$ and $r_{N a}$ (in $\mathrm{g} / \mathrm{m}^{2}$ ) from Quenched and

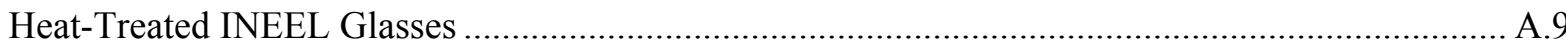

Table A-7. Calculated $r_{B}$ and $r_{N a}$ (in $\mathrm{g} / \mathrm{m}^{2}$ ) from Quenched and Heat-Treated INEEL Glasses........... A.11

Table A-8. Glasses Precipitating Clinopyroxene: $r_{r B}$ and $r_{r N a}$ (in $\mathrm{g} / \mathrm{m}^{2}$ ) Normalized with Respect to Residual Glass............................................................................. A.24

Table A-9. Glasses Precipitating NP: $r_{r B}$ and $r_{r N a}\left(\right.$ in $\mathrm{g} / \mathrm{m}^{2}$ ) Normalized with Respect to Residual Glass.

Table A-10. INEEL Glasses: $r_{r B}$ and $r_{r N a}\left(\right.$ in $\mathrm{g} / \mathrm{m}^{2}$ ) Normalized with Respect to Residual Glass 
PNNL-XXXXX

After this report was finished, a PCT database became available consisting of 51 glasses formulated for Idaho National Environmental Engineering Laboratory (INEEL) high level wastes. Both quenched and heat-treated (CCC) glasses were measured. The crystallinity of CCC glasses was determined using XRD quantitative analysis. This database is analyzed in the section "INEEL Glasses." The section "Renormalized Releases from Heat-Treated Glasses" reinterprets data in terms of PCT releases normalized with respect to calculated compositions of residual glasses.

\section{INEEL Glasses}

The composition region of INEEL glasses differs from those of Savannah River and Hanford in that INEEL glasses have high $\mathrm{CaO}$ and $\mathrm{F}$ concentrations and very low concentrations of $\mathrm{Fe}_{2} \mathrm{O}_{3}$ (Table $\mathrm{A}-1$ ). The PCT component coefficients for this composition region are listed in Table A-1.

The compositions of 51 INEEL glasses are in Table A-2. The minerals detected in INEEL glasses and their effects on PCT are listed in Table A-3 (most of the minerals that precipitated from these glasses are different from those in Table 2.3). Table A-4 lists the mass fractions of minerals in the glasses, and the corresponding compositions of the residual amorphous phase are in Table A-5. Table A-6 and Table A.7 list the measured and calculated PCT releases $\left(r_{B}\right.$ and $\left.r_{N a}\right)$ from quenched and heat-treated glasses. These results are plotted in Figures A-1 to A-10.

The following six out of 51 glasses had more than 10 times higher $r_{h B}$ than $r_{B}$ after quenching (Figure A-1): DZr-CV-2, DZr-CV-5, DP-1, DP-2, DP-6, and DP-23 (Table A-6). These glasses precipitated (Table 2.1) nepheline (NP) with $\ln r_{c B k}=-7.1$ (DZr-CV-2, DP-1, and DP-6), fluorapatite with $\ln r_{c B k}=-4.9$ (DZr-CV-5), baddeleyite with $\ln r_{c B k}=-10.6$ (DP-1), sodium aluminum silicate sulfide with $\ln r_{c B k}=-4.8$ (DZr-CV-2 and DP-2), and calcium fluoride silicate sulfate with $\ln r_{c B k}=-5.7$ (DP-6). Two glasses stand out: DP-2 with most of its mass crystallized and DP-23 with no precipitation that would justify the high $r_{h B}$ and $r_{h N a}$. Two glasses, DZr-CV-4 and DZr-CV-9, displayed substantially lower $r_{h B}$ than $r_{B}$ while precipitating small fractions of minerals that have a negative effect on glass corrosion (cuspidine, fluorapatite, and sodium calcium silicate sulfide). This casts doubt on either PCT-measured values or crystallinity identification, unless other factors are responsible for this aberration. An erroneous value of the $r_{B}$ is likely from DZr-CV-9. Out of 51 glasses, only one, DP-23, exhibited $r_{h N a}$ an order of magnitude higher than $r_{N a}$ (Figure A-2).

Figure A-3 shows good agreement between $r_{B}$ and $r_{N a}$. The only exception is DZr-CV-9 with an unusually high $r_{B}$, probably a measurement error. Slightly higher $r_{N a}$ from most glasses are a consequence of $\mathrm{Na}$ ion exchange at the beginning of the dissolution process (lower values are also possible if $\mathrm{Na}$ is a component of secondary mineral phases). The difference between $r_{h B}$ and $r_{h N a}$ is generally larger than the difference between $r_{B}$ and $r_{N a}$ from quenched glasses (Figure A-4). This may be caused by the change in amorphous phase composition as a result of crystallization (measured $r_{h B}$ and $r_{h N a}$ have not been renormalized to calculated residual glass composition). 
Calculated $r_{B}$ and $r_{N a}$ are in reasonable agreement with measured values (Figure A-5 and Figure A6), DZr-CV-9 being an exception. Calculated $r_{r B}$ and $r_{r N a}$ are displayed in Figures A-7 to A-10. Calculated and measured values are in better agreement when measured $r_{B}$ and $r_{N a}$ are used. Except for two glasses (DP-2 and DP-23), calculated $r_{r B}$ and $\mathrm{r}_{\mathrm{rNa}}$ are in good agreement with measured $r_{h B}$ and $r_{h N a}$ (Figures A-9 and A-10). The very low $\mathrm{r}_{\mathrm{rNa}}$ from heat-treated DP-23 glass is a consequence of a relatively low detected crystallinity fraction of hiortdahlite with $\ln r_{c B k}=-0.3$ and a tiny fraction of lazurite-A (Table A-4). It is possible that crystallinity detection in heat-treated DP-23 glass was in error. Four glasses, DZrCV-2, DZr-CV-5, DP-1, and DP-23, had substantially higher $r_{h B}$ than $r_{r B}$. As discussed above, three of these glasses precipitated minerals that have a negative impact on $r_{B}$, but the crystallinity fraction was not high enough in these glasses to justify the low $r_{h B}$. Two glasses, DZr-CV-4 and DZr-CV-9, had substantially smaller $r_{h B}$ than $r_{r B}$. We suspect that the measured values are in error $\left(r_{h B}\right.$ values were much smaller than $r_{B}$ values, see above).

Table A-1. Component Coefficients for $r_{B}$ and $r_{N a}\left(\ln \text { values for } r_{j} \text { in } \mathbf{g} / \mathbf{m}^{2}\right)^{(1)}$ and Waste Glass Composition Region

\begin{tabular}{|c|c|c|c|c|c|c|c|c|c|c|c||}
\hline & $\mathbf{A l}_{\mathbf{2}} \mathbf{O}_{\mathbf{3}}$ & $\mathbf{B}_{\mathbf{2}} \mathbf{O}_{\mathbf{3}}$ & $\mathbf{C a O}$ & $\mathbf{F}$ & $\mathbf{F e}_{\mathbf{2}} \mathbf{O}_{\mathbf{3}}$ & $\mathbf{L i}_{\mathbf{2}} \mathbf{O}$ & $\mathbf{N a}_{\mathbf{2}} \mathbf{O}$ & $\mathbf{P}_{\mathbf{2}} \mathbf{O}_{\mathbf{5}}$ & $\mathbf{S i O}_{\mathbf{2}}$ & $\mathbf{Z r O}_{\mathbf{2}}$ & Others \\
\hline $\mathrm{b}_{\mathrm{iB}}$ & -25.44 & 11.83 & -9.05 & 0.16 & -3.10 & 22.97 & 17.83 & 0.16 & -4.30 & -10.63 & 0.16 \\
\hline $\mathrm{b}_{\mathrm{iNa}}$ & -25.62 & 9.37 & -2.04 & -0.80 & -4.11 & 19.83 & 19.71 & -0.80 & -4.47 & -11.61 & -0.80 \\
\hline $\max$ & 0.125 & 0.150 & 0.150 & 0.065 & 0.050 & 0.070 & 0.130 & 0.025 & 0.470 & 0.080 & 0.067 \\
\hline $\min$ & 0.070 & 0.047 & 0.095 & 0.040 & 0.000 & 0.045 & 0.080 & 0.000 & 0.360 & 0.040 & 0.010 \\
\hline
\end{tabular}

${ }^{(1)}$ Piepel, G.F., S.K. Cooley, D.K. Peeler, J.D. Vienna, T.B. Edwards. 2000. "Augmenting a Waste Glass Mixture Experiment Study with Additional Glass Components and Experimental Runs," PNNL-SA-33717, Pacific Northwest National Laboratory, Richland, Washington (submitted for publication in Quality Engineering). 
PNNL-XXXXX

Table A-2. Composition of INEEL Glasses (in mass fractions of oxides)

\begin{tabular}{|c|c|c|c|c|c|c|c|c|c|c|c|c|}
\hline Glass & $\overline{\mathrm{I}_{2} \mathrm{O}_{3}}$ & $\mathrm{~B}_{2} \mathbf{O}_{3}$ & $\mathrm{aO}$ & $\mathbf{F}$ & $\mathrm{Fe}_{2} \mathrm{O}_{3}$ & $\mathbf{L i}_{2} \mathbf{O}$ & $\mathrm{Na}_{2} \mathrm{O}$ & $\mid \mathbf{P}_{2} \mathbf{O}_{5}$ & $\mathrm{SiO}_{2}$ & $\mathrm{ZrO}_{2}$ & & \\
\hline DZr-CV-1 & 70 & 52 & 15 & 0. & & 51 & 16 & 12 & 30 & 048 & 18 & 1 \\
\hline $\mathrm{Zr}-\mathrm{CV}-2$ & 25 & 549 & 08 & 0.045 & 0.029 & 0.057 & 0.109 & 0.011 & 0.405 & 0.045 & 017 & 1 \\
\hline-3 & 85 & 660 & 2 & 047 & 330 & 059 & 13 & 12 & 419 & 047 & & \\
\hline-4 & 77 & 50 & 11 & 042 & 27 & 054 & 02 & 10 & 379 & 0.042 & 16 & \\
\hline Zr-CV-5 & 088 & 0.052 & 095 & 0.048 & 031 & 0.061 & 0.116 & 0.012 & 432 & 0.048 & 018 & 1 \\
\hline$T-6$ & 882 & 049 & 150 & 0.045 & 029 & 0.058 & 109 & 0.011 & 405 & 0.045 & U & \\
\hline-7 & 87 & 551 & 4 & 0.040 & 030 & 0.060 & 115 & 012 & 426 & 0.047 & 18 & \\
\hline $\mathrm{Zr}-\mathrm{C}$ & 84 & 550 & 11 & 065 & 029 & 059 & 112 & 011 & 415 & 0.046 & 017 & \\
\hline-9 & 889 & 0.052 & 17 & 0.048 & 0.000 & 0.062 & 0.117 & 0.012 & 0.436 & 0.049 & 018 & \\
\hline-10 & 84 & 50 & & 0.046 & 050 & 0.059 & 111 & 0.011 & 0.414 & 0.046 & 017 & \\
\hline 11 & 84 & 550 & & 046 & 29 & 059 & 111 & 011 & 412 & 046 & 42 & \\
\hline DZr-C & 082 & 0.048 & 0.108 & 0.045 & 028 & 0.057 & 0.108 & 0.011 & 0.402 & 0.045 & .067 & \\
\hline-13 & 887 & 0.052 & 5 & 0.048 & 30 & 0.045 & 0.116 & 0.012 & 0.430 & 0.048 & .018 & \\
\hline 14 & $\pi$ & 50 & & +7 & 0 & 70 & 13 & 12 & 18 & $4 /$ & 11 & \\
\hline $\mathrm{DZr}$ & 39 & 053 & 8 & 0.049 & 31 & 0.062 & 80 & 12 & 0.439 & 0.049 & 18 & \\
\hline-16 & 084 & 0.050 & 111 & 0.046 & 29 & 0.059 & 130 & 0.012 & 0.415 & 0.046 & .017 & 1 \\
\hline 17 & 087 & 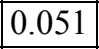 & & ( & 0 & 1 & 5 & 00 & 28 & 0 & .018 & \\
\hline $\mathrm{DZr}$ & 5 & 50 & 2 & 0.046 & 0 & 059 & 12 & 25 & 0.417 & 0.046 & 017 & \\
\hline DZr-C & 086 & 0.051 & 114 & 0.047 & 030 & 0.060 & 114 & 0.012 & 0.425 & 0.047 & .012 & \\
\hline-20 & 6 & 0.051 & 3 & 0.047 & 0 & 0.060 & 13 & 0.012 & 0.421 & 0.047 & 22 & 1 \\
\hline $\mathrm{Zr}-$ & $\tau$ & 66 & 6 & 052 & 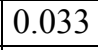 & 0.067 & 126 & 13 & 0.360 & 0.052 & .020 & \\
\hline-22 & 979 & 047 & 04 & 0.043 & 28 & 055 & 104 & 0.011 & 0.470 & 0.043 & 016 & 1 \\
\hline $\mathrm{DZ}$ & 87 & 0.051 & 4 & 0.047 & 30 & 0.060 & 115 & 12 & 0.426 & 0.040 & 018 & 1 \\
\hline $\mathrm{DZ}$ & 33 & 49 & 9 & 0.045 & 29 & 0.058 & 10 & 0.011 & 0.408 & 0.080 & 017 & \\
\hline DP-C & 89 & 0.082 & 14 & 0.051 & 0.018 & 056 & 098 & 0.011 & 0.380 & 0.055 & 046 & \\
\hline DP- & 991 & 991 & 21 & 0. & 5 & 50 & 0.106 & 15 & 0.390 & 0.050 & 10 & 1 \\
\hline DP-BI & 087 & 0.051 & 4 & 0.047 & 0. & 0.060 & 0.115 & 0.012 & 0.426 & 0.047 & 11 & \\
\hline DP-1 & 125 & 060 & 0 & 0.040 & 000 & 0.070 & 0.080 & 0.000 & 0.365 & 0.080 & .030 & \\
\hline DP-2 & 125 & 0.060 & 50 & 0.040 & 10 & 0.045 & 0.130 & 0.000 & 0.360 & 0.040 & .040 & 1 \\
\hline DP-3 & 25 & 0.060 & 5 & 0.065 & 0.050 & 0.070 & 0.080 & 0.000 & 0.375 & 0.040 & 040 & 1 \\
\hline DP-4 & 25 & 060 & 5 & 0.065 & 0.000 & 0.045 & 0.080 & 0.025 & 0.360 & 0.080 & .065 & \\
\hline DP-5 & 125 & 0.060 & 095 & 0.040 & 0.050 & 0.045 & 0.080 & 0.025 & 0.360 & 0.080 & .040 & 1 \\
\hline DP-6 & 125 & 060 & 095 & 0.040 & 0.000 & 0.070 & 0.130 & 0.025 & 0.360 & 0.040 & .055 & 1 \\
\hline DP-7 & 25 & 135 & 095 & 0.040 & 0.000 & 0.070 & 0.080 & 0.000 & 0.360 & 0.040 & .055 & 1 \\
\hline DP-8 & 070 & 0.150 & 35 & 0.065 & 0.000 & 0.045 & 0.080 & 0.025 & 0.360 & 0.080 & \begin{tabular}{|l|}
0.030 \\
\end{tabular} & 1 \\
\hline DP-9 & .070 & 0.150 & 095 & 0.040 & 0.000 & 0.045 & 0.130 & 0.025 & 0.365 & 0.040 & .040 & 1 \\
\hline DP-1C & 970 & 0.150 & 0.100 & 0.065 & 0.000 & 0.070 & 0.080 & 0.000 & 0.360 & 0.040 & .065 & 1 \\
\hline & 70 & 0.150 & 0 & 0.040 & 0.050 & 0.070 & 0.080 & 0.000 & 0.360 & 0.040 & .040 & \\
\hline DP-12 & 0.070 & 0.060 & 0.150 & 0.065 & 0.050 & 0.045 & 0.080 & 0.025 & 0.360 & 0.040 & 0.055 & 1 \\
\hline DP-13 & 70 & 0.060 & 50 & 0.065 & 0.000 & 0.070 & 0.080 & 0.025 & 0.360 & 0.080 & .040 & 1 \\
\hline DP-14 & 0.070 & 0.060 & 0.150 & 0.040 & 0.050 & 0.070 & 0.080 & 0.025 & 0.360 & 0.040 & 0.055 & 1 \\
\hline
\end{tabular}

A. 3 
Table A-2 (Contd)

\begin{tabular}{|c|c|c|c|c|c|c|c|c|c|c|c|c|}
\hline Glass & $\overline{\mathrm{I}_{2} \mathrm{O}_{3}}$ & $\mathrm{~B}_{2} \mathrm{O}_{3}$ & CaO & $\bar{F}$ & $\mathrm{Fe}_{2} \mathrm{O}_{3}$ & \begin{tabular}{|l}
$\mathbf{L i}_{2} \mathbf{O}$ \\
\end{tabular} & $\mathrm{Na}_{2} \mathbf{O}$ & 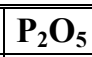 & $\mathbf{S i O}_{2}$ & $\mathrm{ZrO}_{2}$ & Othe & Sum \\
\hline DP-15 & 070 & 0.060 & \begin{tabular}{|l|}
0.150 \\
\end{tabular} & \begin{tabular}{|l|}
0.040 \\
\end{tabular} & 0.000 & 0.045 & \begin{tabular}{|l|}
0.080 \\
\end{tabular} & 0.000 & 0.450 & 0.040 & 0.065 & 1 \\
\hline DP-16 & .070 & 0.060 & 0.095 & 0.065 & 0.050 & 0.045 & 0.080 & 0.000 & 0.465 & 0.040 & 0.030 & 1 \\
\hline DP-17 & .070 & 0.060 & \begin{tabular}{|l|l|}
0.095 \\
\end{tabular} & \begin{tabular}{|l|}
0.065 \\
\end{tabular} & $\mid 0.000$ & $\mid 0.070$ & \begin{tabular}{|l|l|}
0.130 \\
\end{tabular} & 0.025 & \begin{tabular}{|l|}
0.415 \\
\end{tabular} & 0.040 & 0.030 & 1 \\
\hline DP-18 & .070 & 0.060 & 0.095 & 0.040 & 0.050 & 0.045 & \begin{tabular}{|l|}
0.130 \\
\end{tabular} & 0.000 & 0.365 & 0.080 & 0.065 & 1 \\
\hline DP-19 & 0.070 & 0.060 & 0.095 & 0.040 & 0.050 & 0.070 & 0.130 & 0.000 & 0.375 & 0.080 & 0.030 & 1 \\
\hline DP-20 & 0.070 & 0.060 & 0.100 & 0.040 & 0.000 & 0.045 & \begin{tabular}{|l|}
0.080 \\
\end{tabular} & 0.000 & 0.470 & 0.080 & 0.055 & 1 \\
\hline DP-21 & .070 & 0.145 & 0.150 & \begin{tabular}{|l|}
0.040 \\
\end{tabular} & 0.000 & 0.045 & \begin{tabular}{|l|}
0.080 \\
\end{tabular} & 0.000 & 0.360 & 0.080 & 0.030 & 1 \\
\hline DP-22 & \begin{tabular}{|l|l|}
0.070 \\
\end{tabular} & 0.065 & 0.095 & 0.065 & 0.000 & $\mid 0.070$ & \begin{tabular}{|l|}
0.130 \\
\end{tabular} & 0.000 & \begin{tabular}{|l|}
0.360 \\
\end{tabular} & 0.080 & 0.065 & 1 \\
\hline DP-23 & 0.120 & 0.060 & 0.150 & 0.065 & 0.000 & 0.045 & \begin{tabular}{|l|}
0.130 \\
\end{tabular} & 0.000 & 0.360 & 0.040 & 0.030 & 1 \\
\hline DP-24 & \begin{tabular}{|l|}
0.080 \\
\end{tabular} & 0.060 & \begin{tabular}{|l|}
0.095 \\
\end{tabular} & \begin{tabular}{|l|}
0.040 \\
\end{tabular} & 0.000 & \begin{tabular}{|l|}
0.070 \\
\end{tabular} & \begin{tabular}{|l|}
0.080 \\
\end{tabular} & 0.025 & \begin{tabular}{|l}
0.470 \\
\end{tabular} & \begin{tabular}{|l|}
0.040 \\
\end{tabular} & 0.040 & 1 \\
\hline
\end{tabular}

Table A-3. Minerals and their Effects on $r_{B}$ and $r_{N a}$ (ln values for $r_{j}$ in $g / \mathbf{m}^{2}$ ) from INEEL Glasses

\begin{tabular}{|c|c|c|c|c|c|c|c|c|c|c|c|}
\hline Mineral & $\mathbf{A l}_{\mathbf{2}} \mathbf{O}_{3}$ & $\mathrm{CaO}$ & $\mathbf{F}$ & $\mathbf{L i}_{2} \mathbf{O}$ & $\mathrm{Na}_{2} \mathrm{O}$ & $\mathbf{P}_{2} \mathrm{O}_{5}$ & $\mathrm{SiO}_{2}$ & $\mathrm{ZrO}_{2}$ & Others & $\ln r_{\mathrm{cBk}}$ & $\ln r_{\mathrm{cNak}}$ \\
\hline Apatite & & 0.547 & 0.037 & & & 0.416 & & & & -4.88 & -1.48 \\
\hline $\begin{array}{l}\text { Baddeleyite } \\
\text { (Zirconium Oxide) }\end{array}$ & & & & & & & & 1.000 & & -10.63 & -11.61 \\
\hline $\begin{array}{l}\text { Calcium Fluoride } \\
\text { Silicate Sulfate }\end{array}$ & & 0.550 & 0.037 & & & & 0.177 & & 0.236 & -5.69 & -2.13 \\
\hline Cuspidine & & 0.507 & 0.086 & & & & 0.407 & & & -6.32 & -2.92 \\
\hline Fluorapatite & & 0.547 & 0.037 & & & 0.416 & & & & -4.88 & -1.48 \\
\hline Fluorellestadite & & 0.550 & 0.037 & & & & 0.177 & & 0.236 & -5.69 & -2.13 \\
\hline $\begin{array}{l}\text { Fluorite } \\
\text { (Calcium Fluoride) }\end{array}$ & & 0.596 & 0.404 & & & & & & & -5.33 & -1.54 \\
\hline Hiortdahlite & & 0.140 & 0.105 & & 0.180 & & 0.332 & 0.177 & 0.066 & -1.33 & -0.41 \\
\hline Lazurite-A & 0.272 & 0.100 & & & 0.165 & & 0.321 & & 0.142 & -6.23 & -5.46 \\
\hline Lithium Silicate & & & & 0.332 & & & 0.668 & & & 4.76 & 3.60 \\
\hline Nepheline & 0.359 & & & & 0.218 & & 0.423 & & & -7.06 & -6.79 \\
\hline Nosean & 0.308 & & & & 0.249 & & 0.363 & & 0.081 & -4.93 & -4.65 \\
\hline Sodalite & 0.321 & & 0.040 & & 0.260 & & 0.379 & & & -5.15 & -4.82 \\
\hline $\begin{array}{l}\text { Sodium Aluminum } \\
\text { Silicate Sulfide }\end{array}$ & 0.272 & & & & 0.193 & & 0.321 & & 0.214 & -4.83 & -4.78 \\
\hline $\begin{array}{l}\text { Sodium Calcium } \\
\text { Fluoride Sulfate }\end{array}$ & & 0.277 & 0.047 & & 0.230 & & 0.446 & & & -0.32 & 1.94 \\
\hline $\begin{array}{l}\text { Sodium Calcium } \\
\text { Oxide Fluoride } \\
\text { Phosphate }\end{array}$ & & 0.336 & 0.114 & & 0.124 & 0.426 & & & & -0.75 & 1.32 \\
\hline Zircon & & & & & & & 0.397 & 0.603 & & -8.12 & -8.77 \\
\hline Zirconium Titanium Oxide & & & & & & & & 0.533 & 0.467 & -5.59 & -6.56 \\
\hline
\end{tabular}


PNNL-XXXXX

Table A-4. Crystallinity (in mass fractions of minerals) of Heat-Treated INEEL Glasses

\begin{tabular}{|c|c|c|c|c|c|c|c|c|c|c|}
\hline $\begin{array}{l}\frac{\omega}{\omega} \\
\frac{\omega}{\omega}\end{array}$ & 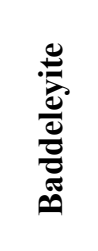 & שֶ: & 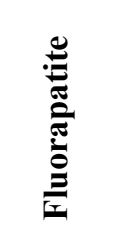 & 苛 & 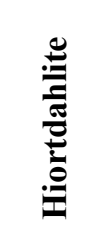 & 芯 & 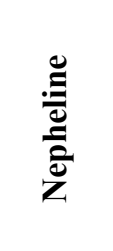 & 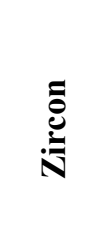 & 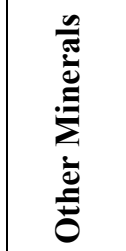 & है \\
\hline DZr-CV-1 & & & & & & & & & & 0.000 \\
\hline DZr-CV-2 & & & & & 0.204 & & 0.035 & & $0.131^{(7)}$ & 0.370 \\
\hline DZr-CV-3 & & & 0.008 & 0.004 & & & & & & 0.012 \\
\hline DZr-CV-4 & & 0.062 & & & & & & & $0.021^{(7)}$ & 0.083 \\
\hline DZr-CV-5 & & & 0.017 & & & & & & & 0.017 \\
\hline DZr-CV-6 & & & & & & & & & & 0.000 \\
\hline DZr-CV-7 & & & & & & & & & & 0.000 \\
\hline DZr-CV-8 & & & 0.003 & 0.038 & & & & & & 0.042 \\
\hline DZr-CV-9 & & & 0.021 & & & & & & & 0.021 \\
\hline DZr-CV-10 & & 0.030 & & & & & & & & 0.030 \\
\hline DZr-CV-11 & & 0.016 & & & & & & & & 0.016 \\
\hline DZr-CV-12 & & & & & & & & & $0.055^{(1)}$ & 0.055 \\
\hline DZr-CV-13 & & & & 0.014 & & & & & $0.062^{(9)}$ & 0.077 \\
\hline DZr-CV-14 & & & & & & & & & & 0.000 \\
\hline DZr-CV-15 & & & & 0.024 & & & & & & 0.024 \\
\hline DZr-CV-16 & & & & & & & & & & 0.000 \\
\hline DZr-CV-17 & & 0.013 & & & & & & & & 0.013 \\
\hline DZr-CV-18 & & & 0.048 & & & & & & & 0.048 \\
\hline DZr-CV-19 & & & & & & & & & & 0.000 \\
\hline DZr-CV-20 & & & & & & & & & $0.087^{(6)}$ & 0.087 \\
\hline DZr-CV-21 & & 0.037 & & & & & & & $0.121^{(5)}$ & 0.157 \\
\hline DZr-CV-22 & & & 0.006 & 0.006 & & & & & & 0.013 \\
\hline DZr-CV-23 & & 0.010 & & & & & & & & 0.010 \\
\hline DZr-CV-24 & & & & 0.006 & 0.153 & & & & & 0.159 \\
\hline DP-Centroid & 0.007 & & & 0.006 & & & & & & 0.013 \\
\hline DP-BL1 & & & & & & & & & $0.053^{(8)}$ & 0.053 \\
\hline DP-BL2 & & & & & & & & & & 0.000 \\
\hline DP-1 & 0.048 & & & & & & 0.229 & & $0.055^{(4)}$ & 0.332 \\
\hline DP-2 & & & & & 0.333 & & & & $0.372^{(7)}$ & 0.704 \\
\hline DP-3 & & & & 0.066 & & & & & $0.170^{(7)}$ & 0.236 \\
\hline DP-4 & 0.085 & & & 0.046 & & & & 0.101 & & 0.232 \\
\hline DP-5 & 0.104 & & 0.089 & 0.050 & & & 0.049 & & & 0.294 \\
\hline DP-6 & & & & & & & 0.028 & & $0.182^{(2)}$ & 0.209 \\
\hline DP-7 & & & & & & & & & & 0.000 \\
\hline
\end{tabular}


Table A-4 (Contd)

\begin{tabular}{|l|l|l|l|l|l|l|l|l|l||}
\hline & & & & & & & & & \\
\hline
\end{tabular}

${ }^{(1)}$ Apatite

${ }^{(2)}$ Calcium Fluoride Silicate Sulfate

${ }^{(3)}$ Fluorellestadite

${ }^{(4)}$ Lithium Silicate

${ }^{(5)}$ Nosean

${ }^{(6)}$ Sodalite

${ }^{(7)}$ Sodium Aluminum Silicate Sulfide

${ }^{(8)}$ Sodium Calcium Fluoride Sulfate

${ }^{(9)}$ Sodium Calcium Oxide Fluoride Phosphate

${ }^{(10)}$ Zirconium Titanium Oxide 
PNNL-XXXXX

Table A-5. Compositions of INEEL Residual Glasses (in mass fractions of oxides)

\begin{tabular}{|c|c|c|c|c|c|c|c|c|c|c|c|c|}
\hline Glass & $\mathrm{I}_{2} \mathrm{O}_{3}$ & $\mathbf{B}_{2} \mathbf{O}_{3}$ & $\mathrm{CaO}$ & $\mathbf{F}$ & $\overline{\mathrm{Fe}_{2} \mathrm{O}_{3}}$ & $\mathbf{L i}_{2} \mathbf{O}$ & $\mathrm{Na}_{2} \mathrm{O}$ & $\mathbf{P}_{2} \mathbf{O}_{5}$ & $\mathrm{SiO}_{2}$ & $\mathrm{ZrO}_{2}$ & & \\
\hline DZr-CV-1 & 070 & 052 & 115 & 0.048 & 030 & 0.061 & 116 & 012 & 430 & .048 & 0.018 & 1 \\
\hline DZr-CV-2 & 122 & 077 & 127 & 0.037 & 0.046 & 0.091 & 062 & .018 & 0.445 & 0.014 & ת & \\
\hline $\mathrm{DZr}$ & 86 & 060 & & 047 & 30 & 0.060 & 14 & 08 & 422 & 047 & & \\
\hline DZr-CV-4 & 978 & 164 & 76 & 0.040 & 0.029 & 0.059 & 107 & 011 & 378 & 0.046 & & \\
\hline DZr-CV-5 & .089 & 0.053 & 087 & 0.048 & 0.031 & 0.062 & 0.118 & 0.005 & 0.439 & 0.049 & .018 & 1 \\
\hline$\overline{\mathrm{Zr}-}$ & 082 & 049 & 150 & 0.045 & 029 & 0.058 & 109 & 011 & .405 & .045 & & \\
\hline DZr- & 887 & 051 & 114 & 0.040 & 030 & 0.060 & 115 & 012 & 0.426 & 0.047 & 18 & \\
\hline DZr-CV-8 & 088 & 052 & 092 & 0.051 & 031 & 0.061 & 116 & 012 & 431 & .048 & 018 & \\
\hline DZr-CV-9 & 090 & 054 & 108 & $\mid 0.049$ & 0.000 & 0.063 & 120 & 0.004 & .445 & .050 & & 1 \\
\hline DZr-CV-10 & 887 & 051 & 099 & 0.045 & 052 & 0.061 & 115 & 012 & 0.414 & .048 & & \\
\hline DZr-C & 085 & 050 & 04 & 0.045 & 030 & 0.059 & 113 & 012 & 0.412 & .047 & & \\
\hline DZr-CV-12 & 0.086 & 0.051 & 082 & 0.045 & 0.030 & 0.060 & 0.114 & -0.013 & 0.425 & 0.047 & & 1 \\
\hline DZr-CV-13 & 0.095 & 056 & 093 & 0.038 & 0.033 & 0.049 & \begin{tabular}{|l|}
0.117 \\
\end{tabular} & -0.016 & 0.465 & 0.052 & 19 & 1 \\
\hline DZr- & 85 & 50 & 12 & 0.047 & 30 & 0.070 & 13 & 12 & 418 & 47 & & \\
\hline$\overline{D Z r-C}$ & 093 & 055 & 097 & 0.040 & 33 & 0.065 & 084 & 004 & 459 & .051 & & 1 \\
\hline DZr-CV-16 & 0.084 & 0.050 & 111 & 0.046 & 0.029 & 0.059 & 0.130 & 0.012 & 0.415 & 0.046 & 17 & 1 \\
\hline DZr- & 088 & 52 & 09 & 0.047 & 1 & 0.061 & 117 & 00 & 28 & 48 & & \\
\hline DZr- & 089 & 953 & 090 & 0.047 & 31 & 0.062 & 118 & 005 & .438 & 0.049 & & 1 \\
\hline DZr-CV-19 & 0.086 & 0.051 & 114 & 0.047 & 0.030 & 0.060 & 114 & .012 & 0.425 & .047 & & 1 \\
\hline DZr-CV-20 & 0.063 & 055 & 123 & \begin{tabular}{|l|}
0.047 \\
\end{tabular} & 0.033 & 0.065 & 099 & 13 & 0.425 & 0.051 & 0.024 & 1 \\
\hline DZr-C & .069 & 067 & 127 & 0.058 & 0.039 & 0.079 & 0.114 & 015 & 0.358 & 0.062 & & \\
\hline DZr-CV-22 & .080 & \begin{tabular}{|l|}
0.047 \\
\end{tabular} & 098 & $\mid 0.041$ & 0.028 & 0.056 & 0.106 & 008 & 0.476 & .044 & & 1 \\
\hline $\mathrm{DZr}-$ & 0.087 & 052 & 110 & 0.047 & 0.031 & 0.061 & 116 & 12 & 0.426 & 0.040 & & 1 \\
\hline DZr-CV-24 & .099 & 58 & 101 & 0.032 & 0.034 & 0.069 & 0.098 & 0.013 & 0.425 & \begin{tabular}{|l|}
0.063 \\
\end{tabular} & 0.008 & 1 \\
\hline DP-Centroid & 090 & 0.083 & 112 & 0.049 & 0.018 & 0.057 & 0.100 & 0.011 & 0.385 & 0.049 & ro & 1 \\
\hline DP-BL1 & .096 & 0.096 & 112 & 0.051 & 0.027 & 0.053 & 0.099 & .016 & 0.387 & .053 & & 1 \\
\hline DP-BL2 & \begin{tabular}{|l|}
0.087 \\
\end{tabular} & 0.051 & 114 & 0.047 & 0.030 & 0.060 & 0.115 & 0.012 & 0.426 & 0.047 & 1 & 1 \\
\hline DP-1 & 064 & 0.090 & 225 & 0.060 & 0.000 & 0.077 & 0.045 & 0.000 & 0.346 & \begin{tabular}{|l|}
0.048 \\
\end{tabular} & J & 1 \\
\hline DP-2 & .080 & 0.203 & 350 & \begin{tabular}{|l|}
0.017 \\
\end{tabular} & 0.034 & 0.152 & -0.006 & 0.000 & 0.440 & -0.063 & .208 & 1 \\
\hline DP-3 & 0.103 & 0.079 & 0.073 & 0.050 & 0.065 & 0.092 & 0.062 & \begin{tabular}{|l|l|}
0.000 \\
\end{tabular} & 0.419 & \begin{tabular}{|l|}
0.052 \\
\end{tabular} & & 1 \\
\hline DP-4 & 144 & 0.069 & 078 & 0.054 & 0.000 & 0.052 & \begin{tabular}{|l|}
0.092 \\
\end{tabular} & \begin{tabular}{|l|}
0.029 \\
\end{tabular} & 0.414 & -0.006 & & 1 \\
\hline DP-5 & 152 & 0.085 & 0.023 & 0.023 & 0.071 & 0.064 & \begin{tabular}{|l|}
0.098 \\
\end{tabular} & -0.017 & 0.480 & -0.034 & 0. & 1 \\
\hline DP-6 & 0.146 & 0.076 & -0.006 & 0.042 & 0.000 & 0.089 & 0.157 & 0.032 & 0.400 & 0.051 & 15 & 1 \\
\hline DP-7 & 125 & 0.135 & 0.095 & 0.040 & 0.000 & 0.070 & 0.080 & $\mid 0.000$ & 0.360 & 0.040 & 55 & 1 \\
\hline DP-8 & 081 & 0.174 & 0.029 & 0.047 & 0.000 & 0.052 & \begin{tabular}{|l|}
0.093 \\
\end{tabular} & -0.004 & 0.410 & \begin{tabular}{|l|}
0.082 \\
\end{tabular} & 35 & 1 \\
\hline DP-9 & 0.075 & 0.162 & 0.060 & 0.040 & 0.000 & 0.049 & 0.140 & -0.005 & 0.393 & 0.043 & .043 & 1 \\
\hline DP-10 & 0.081 & 0.173 & 026 & 0.017 & 0.000 & 0.081 & 0.092 & -0.002 & 0.416 & \begin{tabular}{|l|}
0.043 \\
\end{tabular} & 072 & 1 \\
\hline DP-11 & 070 & 0.150 & 0.100 & 0.040 & 0.050 & 0.070 & \begin{tabular}{|l|}
0.080 \\
\end{tabular} & 0.000 & 0.360 & 0.040 & 0.040 & 1 \\
\hline DP-12 & .070 & 0.060 & 0.150 & 0.065 & 0.050 & 0.045 & \begin{tabular}{|l|}
0.080 \\
\end{tabular} & 0.025 & 0.360 & 0.040 & .055 & 1 \\
\hline DP-13 & 82 & 0.087 & 0.144 & 0.069 & 0.000 & 0.102 & 0.100 & 0.003 & 0.396 & -0.041 & .058 & 1 \\
\hline DP-14 & 0.085 & 0.073 & 0.064 & 0.041 & 0.061 & 0.085 & 0.097 & \begin{tabular}{|l|l|}
0.030 \\
\end{tabular} & 0.399 & \begin{tabular}{|l|}
0.049 \\
\end{tabular} & 0.016 & 1 \\
\hline
\end{tabular}


PNNL-XXXXX

Table A-5 (Contd)

\begin{tabular}{|l|c|c|c|c|c|c|c|c|c|c|c|c||}
\hline \hline Glass & $\mathbf{A l}_{2} \mathbf{O}_{3}$ & $\mathbf{B}_{2} \mathbf{O}_{3}$ & $\mathbf{C a O}$ & $\mathbf{F}$ & $\mathbf{F e}_{2} \mathbf{O}_{3}$ & $\mathbf{L i}_{2} \mathbf{O}$ & $\mathbf{N a}_{2} \mathbf{O}$ & $\mathbf{P}_{\mathbf{2}} \mathbf{O}_{\mathbf{5}}$ & $\mathbf{S i O}_{2}$ & $\mathbf{Z r O}_{2}$ & Others & $\mathbf{S u m}$ \\
\hline DP-15 & 0.072 & 0.062 & 0.138 & 0.039 & 0.000 & 0.046 & 0.083 & 0.000 & 0.451 & 0.041 & 0.067 & 1 \\
\hline DP-16 & 0.075 & 0.065 & 0.057 & 0.039 & 0.054 & 0.048 & 0.086 & 0.000 & 0.500 & 0.043 & 0.032 & 1 \\
\hline DP-17 & 0.075 & 0.064 & 0.062 & 0.067 & 0.000 & 0.075 & 0.140 & -0.004 & 0.446 & 0.043 & 0.032 & 1 \\
\hline DP-18 & 0.074 & 0.064 & 0.101 & 0.042 & 0.053 & 0.048 & 0.138 & 0.000 & 0.387 & 0.025 & 0.069 & 1 \\
\hline DP-19 & 0.073 & 0.063 & 0.100 & 0.042 & 0.052 & 0.073 & 0.136 & 0.000 & 0.393 & 0.035 & 0.031 & 1 \\
\hline DP-20 & 0.076 & 0.065 & 0.108 & 0.043 & 0.000 & 0.049 & 0.086 & 0.000 & 0.476 & 0.038 & 0.059 & 1 \\
\hline DP-21 & 0.070 & 0.145 & 0.150 & 0.040 & 0.000 & 0.045 & 0.080 & 0.000 & 0.360 & 0.080 & 0.030 & 1 \\
\hline DP-22 & 0.048 & 0.078 & 0.100 & 0.078 & 0.000 & 0.076 & 0.134 & 0.000 & 0.374 & 0.053 & 0.059 & 1 \\
\hline DP-23 & 0.144 & 0.073 & 0.153 & 0.057 & 0.000 & 0.055 & 0.119 & 0.000 & 0.366 & 0.011 & 0.021 & 1 \\
\hline DP-24 & 0.088 & 0.066 & 0.051 & 0.040 & 0.000 & 0.077 & 0.088 & -0.013 & 0.516 & 0.044 & 0.044 & 1 \\
\hline
\end{tabular}


PNNL-XXXXX

Table A-6. Measured $r_{B}$ and $r_{N a}$ (in $\left.\mathrm{g} / \mathrm{m}^{2}\right)$ from Quenched and Heat-Treated INEEL Glasses

\begin{tabular}{|l|c|c|c|c|}
\hline Glass & $\mathbf{Q}, \mathbf{M}$ & $\mathbf{Q}, \mathbf{M}$ & $\mathbf{H}, \mathbf{M}$ & $\mathbf{H}, \mathbf{M}$ \\
\hline & $\boldsymbol{r}_{\boldsymbol{B}}$ & $\boldsymbol{r}_{\boldsymbol{N} \boldsymbol{a}}$ & $\boldsymbol{r}_{\boldsymbol{h} \boldsymbol{B}}$ & $\boldsymbol{r}_{\boldsymbol{h N a}}$ \\
\hline DZr-CV-1 & 0.309 & 0.387 & 0.218 & 0.311 \\
\hline DZr-CV-2 & 0.107 & 0.216 & 3.119 & 0.977 \\
\hline DZr-CV-3 & 0.207 & 0.303 & 0.165 & 0.282 \\
\hline DZr-CV-4 & 0.896 & 0.842 & 0.059 & 0.308 \\
\hline DZr-CV-5 & 0.210 & 0.321 & 2.243 & 0.601 \\
\hline DZr-CV-6 & 0.131 & 0.263 & 0.177 & 0.242 \\
\hline DZr-CV-7 & 0.172 & 0.300 & 0.125 & 0.237 \\
\hline DZr-CV-8 & 0.183 & 0.294 & 0.233 & 0.318 \\
\hline DZr-CV-9 & 6.561 & 0.338 & 0.164 & 0.285 \\
\hline DZr-CV-10 & 0.215 & 0.337 & 0.156 & 0.263 \\
\hline DZr-CV-11 & 0.206 & 0.337 & 0.134 & 0.251 \\
\hline DZr-CV-12 & 0.206 & 0.319 & 0.106 & 0.244 \\
\hline DZr-CV-13 & 0.098 & 0.213 & 0.118 & 0.216 \\
\hline DZr-CV-14 & 0.237 & 0.396 & 0.182 & 0.327 \\
\hline DZr-CV-15 & 0.102 & 0.164 & 0.096 & 0.143 \\
\hline DZr-CV-16 & 0.217 & 0.398 & 0.151 & 0.322 \\
\hline DZr-CV-17 & 0.110 & 0.261 & 0.077 & 0.228 \\
\hline DZr-CV-18 & 0.236 & 0.375 & 0.178 & 0.269 \\
\hline DZr-CV-19 & 0.125 & 0.266 & 0.096 & 0.219 \\
\hline DZr-CV-20 & 0.062 & 0.133 & 0.057 & 0.239 \\
\hline DZr-CV-21 & 0.310 & 0.483 & 0.659 & 0.574 \\
\hline DZr-CV-22 & 0.118 & 0.234 & 0.105 & 0.204 \\
\hline DZr-CV-23 & 0.140 & 0.288 & 0.126 & 0.255 \\
\hline DZr-CV-24 & 0.141 & 0.251 & 0.130 & 0.218 \\
\hline DP-Centroid & 0.183 & 0.250 & 0.200 & 0.222 \\
\hline DP-BL1 & 0.162 & 0.246 & 0.268 & 0.225 \\
\hline DP-BL2 & 0.161 & 0.274 & 0.219 & 0.245 \\
\hline DP-1 & 0.089 & 0.178 & 7.382 & 0.908 \\
\hline DP-2 & 0.127 & 0.290 & 2.597 & 0.744 \\
\hline DP-3 & 0.102 & 0.154 & 0.355 & 0.270 \\
\hline DP-4 & 0.215 & 0.147 & 0.659 & 0.513 \\
\hline DP-5 & 0.116 & 0.116 & 0.308 & 0.151 \\
\hline DP-6 & 0.413 & 0.560 & 1.049 & 0.804 \\
\hline DP-7 & 0.217 & 0.217 & 0.214 & 0.199 \\
\hline DP-8 & 1.447 & 0.840 & 1.293 & 0.933 \\
\hline DP-9 & 2.033 & 1.795 & 1.923 & 1.766 \\
\hline DP-10 & 0.541 & 0.409 & 0.794 & 0.523 \\
\hline DP-11 & 1.011 & 0.904 & 0.992 & 0.856 \\
\hline
\end{tabular}


PNNL-XXXXX

Table A-6 (Contd)

\begin{tabular}{|l|c|c|c|c||}
\hline \hline Glass & $\mathbf{Q}, \mathbf{M}$ & $\mathbf{Q}, \mathbf{M}$ & $\mathbf{H}, \mathbf{M}$ & $\mathbf{H}, \mathbf{M}$ \\
\hline & $\boldsymbol{r}_{\boldsymbol{B}}$ & $\boldsymbol{r}_{\boldsymbol{N} \boldsymbol{a}}$ & $\boldsymbol{r}_{\boldsymbol{h} \boldsymbol{B}}$ & $\boldsymbol{r}_{\boldsymbol{h N a}}$ \\
\hline DP-12 & 0.128 & 0.218 & 0.218 & 0.228 \\
\hline DP-13 & 0.240 & 0.296 & 0.473 & 0.661 \\
\hline DP-14 & 0.261 & 0.354 & 0.245 & 0.281 \\
\hline DP-15 & 0.104 & 0.198 & - & 0.189 \\
\hline DP-16 & 0.164 & 0.141 & 0.181 & 0.141 \\
\hline DP-17 & 0.735 & 0.854 & 0.559 & 0.730 \\
\hline DP-18 & 0.232 & 0.387 & 0.206 & 0.346 \\
\hline DP-19 & 0.525 & 0.746 & 0.622 & 0.807 \\
\hline DP-20 & 0.123 & 0.203 & - & 0.166 \\
\hline DP-21 & 0.252 & 0.276 & 0.229 & 0.243 \\
\hline DP-22 & 0.421 & 0.607 & 0.833 & 0.767 \\
\hline DP-23 & 0.128 & 0.277 & 7.973 & 2.731 \\
\hline DP-24 & 0.133 & 0.156 & 0.351 & 0.170 \\
\hline
\end{tabular}

A. 10 
PNNL-XXXXX

Table A-7. Calculated $r_{B}$ and $r_{N a}\left(\right.$ in $\left.\mathbf{g} / \mathrm{m}^{2}\right)$ from Quenched and Heat-Treated INEEL Glasses

\begin{tabular}{|c|c|c|c|c|c|c|}
\hline Glass & $\mathbf{Q}, \mathbf{C}$ & $\mathbf{Q}, \mathbf{C}$ & H, QC, RC & H, QC, RC & $\begin{array}{c}\text { H, QM, } \\
\text { RC }\end{array}$ & $\begin{array}{c}\text { H, QM, } \\
\text { RC }\end{array}$ \\
\hline & $r_{B}$ & $\boldsymbol{r}_{\mathrm{Na}}$ & $\boldsymbol{r}_{r B}$ & $\boldsymbol{r}_{r N a}$ & $\boldsymbol{r}_{r B}$ & $r_{r N a}$ \\
\hline & & & \multicolumn{2}{|c|}{ with $r_{j}$ calculated } & \multicolumn{2}{|c|}{ with $r_{j}$ measured } \\
\hline DZr-CV-1 & 0.305 & 0.488 & 0.305 & 0.488 & 0.309 & 0.387 \\
\hline DZr-CV-2 & 0.073 & 0.112 & 0.098 & 0.193 & 0.181 & 0.551 \\
\hline DZr-CV-3 & 0.229 & 0.352 & 0.235 & 0.363 & 0.213 & 0.313 \\
\hline DZr-CV-4 & 0.818 & 0.954 & 1.377 & 1.628 & 1.520 & 1.419 \\
\hline DZr-CV-5 & 0.235 & 0.324 & 0.249 & 0.346 & 0.222 & 0.343 \\
\hline DZr-CV-6 & 0.148 & 0.307 & 0.148 & 0.307 & 0.131 & 0.263 \\
\hline DZr-CV-7 & 0.199 & 0.317 & 0.199 & 0.317 & 0.172 & 0.300 \\
\hline DZr-CV-8 & 0.208 & 0.320 & 0.242 & 0.379 & 0.212 & 0.346 \\
\hline DZr-CV-9 & 0.211 & 0.349 & 0.226 & 0.378 & 7.572 & 0.366 \\
\hline DZr-CV-10 & 0.195 & 0.299 & 0.226 & 0.351 & 0.250 & 0.396 \\
\hline DZr-CV-11 & 0.210 & 0.321 & 0.227 & 0.349 & 0.223 & 0.366 \\
\hline DZr-CV-12 & 0.220 & 0.324 & 0.268 & 0.403 & 0.250 & 0.398 \\
\hline DZr-CV-13 & 0.136 & 0.228 & 0.132 & 0.230 & 0.092 & 0.215 \\
\hline DZr-CV-14 & 0.261 & 0.398 & 0.261 & 0.398 & 0.237 & 0.396 \\
\hline DZr-CV-15 & 0.096 & 0.144 & 0.109 & 0.166 & 0.116 & 0.191 \\
\hline DZr-CV-16 & 0.287 & 0.466 & 0.287 & 0.466 & 0.217 & 0.398 \\
\hline DZr-CV-17 & 0.197 & 0.317 & 0.209 & 0.339 & 0.116 & 0.278 \\
\hline DZr-CV-18 & 0.206 & 0.320 & 0.243 & 0.385 & 0.280 & 0.456 \\
\hline DZr-CV-19 & 0.199 & 0.318 & 0.199 & 0.318 & 0.125 & 0.266 \\
\hline DZr-CV-20 & 0.203 & 0.319 & 0.285 & 0.467 & 0.078 & 0.179 \\
\hline DZr-CV-21 & 0.270 & 0.457 & 0.564 & 1.053 & 0.664 & 1.126 \\
\hline DZr-CV-22 & 0.161 & 0.242 & 0.168 & 0.254 & 0.123 & 0.245 \\
\hline DZr-CV-23 & 0.215 & 0.344 & 0.226 & 0.363 & 0.146 & 0.304 \\
\hline DZr-CV-24 & 0.147 & 0.222 & 0.136 & 0.220 & 0.129 & 0.255 \\
\hline DP-Centroid & 0.211 & 0.299 & 0.230 & 0.328 & 0.199 & 0.273 \\
\hline DP-BL1 & 0.209 & 0.323 & 0.195 & 0.309 & 0.149 & 0.232 \\
\hline DP-BL2 & 0.199 & 0.317 & 0.199 & 0.317 & 0.161 & 0.274 \\
\hline DP-1 & 0.041 & 0.074 & 0.135 & 0.334 & 0.438 & 1.231 \\
\hline DP-2 & 0.085 & 0.188 & 0.461 & 6.866 & 1.815 & 29.521 \\
\hline DP-3 & 0.084 & 0.100 & 0.183 & 0.229 & 0.235 & 0.400 \\
\hline DP-4 & 0.039 & 0.048 & 0.090 & 0.116 & 0.641 & 0.415 \\
\hline DP-5 & 0.033 & 0.041 & 0.171 & 0.232 & 1.010 & 1.010 \\
\hline DP-6 & 0.257 & 0.349 & 0.849 & 1.250 & 1.549 & 2.274 \\
\hline DP-7 & 0.255 & 0.268 & 0.255 & 0.268 & 0.217 & 0.217 \\
\hline DP-8 & 0.456 & 0.474 & 0.955 & 1.000 & 3.647 & 1.942 \\
\hline DP-9 & 1.660 & 2.000 & 2.523 & 3.085 & 3.140 & 2.745 \\
\hline DP-10 & 1.185 & 1.216 & 2.794 & 2.878 & 1.127 & 0.816 \\
\hline
\end{tabular}


PNNL-XXXXX

Table A-7 (Contd)

\begin{tabular}{|c|c|c|c|c|c|c|}
\hline Glass & $\mathbf{Q}, \mathbf{C}$ & $\mathbf{Q}, \mathbf{C}$ & H, QC, RC & $\mathbf{H}, \mathbf{Q C}, \mathbf{R C}$ & $\begin{array}{c}\text { H, QM, } \\
\text { RC } \\
\end{array}$ & $\begin{array}{c}\text { H, QM, } \\
\text { RC }\end{array}$ \\
\hline & $r_{B}$ & $r_{N a}$ & $r_{r B}$ & $\boldsymbol{r}_{r N a}$ & $r_{r B}$ & $\boldsymbol{r}_{r N a}$ \\
\hline & & & \multicolumn{2}{|c|}{ with $r_{j}$ calculated } & \multicolumn{2}{|c|}{ with $r_{j}$ measured } \\
\hline DP-11 & 1.007 & 1.031 & 1.007 & 1.031 & 1.011 & 0.904 \\
\hline DP-12 & 0.126 & 0.232 & 0.126 & 0.232 & 0.128 & 0.218 \\
\hline DP-13 & 0.170 & 0.297 & 1.663 & 3.739 & 2.749 & 3.716 \\
\hline DP-14 & 0.222 & 0.388 & 0.547 & 1.076 & 0.665 & 0.962 \\
\hline DP-15 & 0.099 & 0.196 & 0.113 & 0.229 & 0.118 & 0.231 \\
\hline DP-16 & 0.130 & 0.169 & 0.168 & 0.221 & 0.215 & 0.182 \\
\hline DP-17 & 0.822 & 1.116 & 1.162 & 1.614 & 1.031 & 1.212 \\
\hline DP-18 & 0.320 & 0.440 & 0.566 & 0.792 & 0.403 & 0.691 \\
\hline DP-19 & 0.542 & 0.711 & 0.887 & 1.180 & 0.859 & 1.241 \\
\hline DP-20 & 0.093 & 0.126 & 0.147 & 0.204 & 0.198 & 0.342 \\
\hline DP-21 & 0.259 & 0.421 & 0.259 & 0.421 & 0.252 & 0.276 \\
\hline DP-22 & 0.723 & 0.933 & 2.166 & 2.936 & 1.135 & 1.757 \\
\hline DP-23 & 0.099 & 0.220 & 0.084 & 0.221 & 0.113 & 0.292 \\
\hline DP-24 & 0.206 & 0.255 & 0.284 & 0.360 & 0.176 & 0.210 \\
\hline
\end{tabular}


PNNL-XXXXX

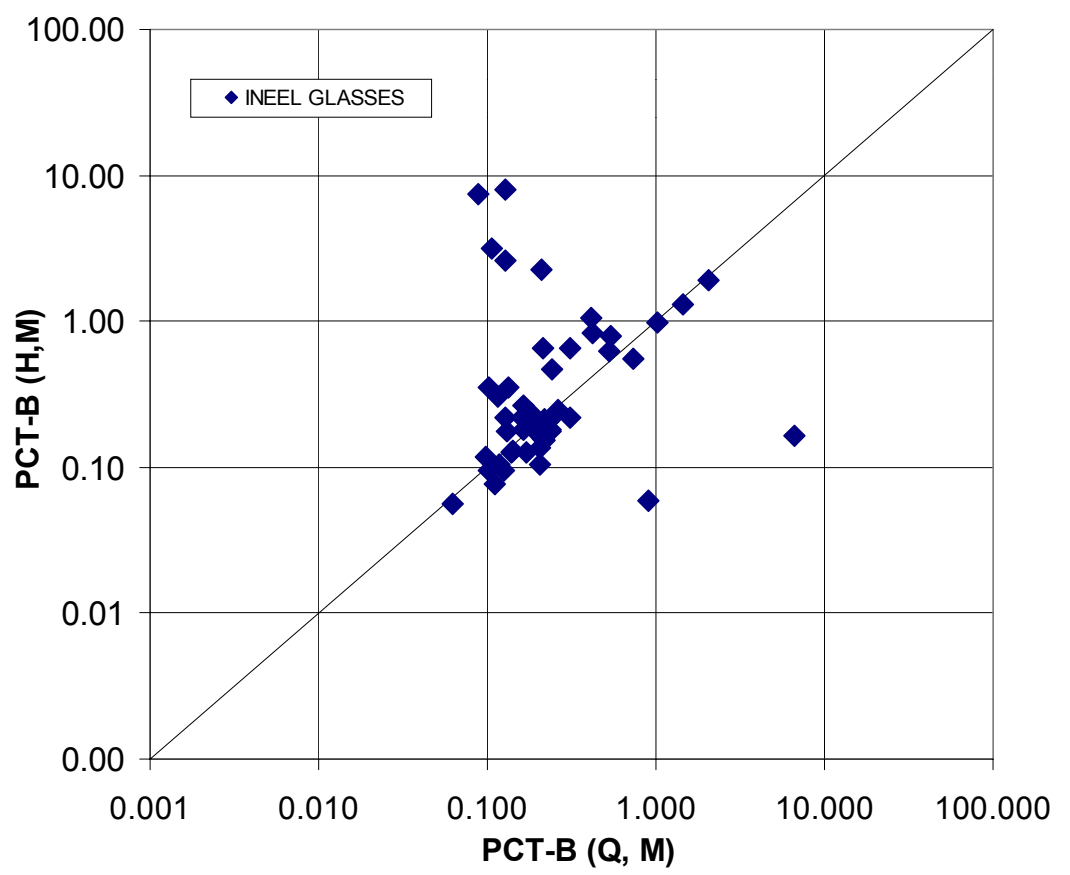

Figure A-1. INEEL Glasses: $r_{h B}$ vs. $r_{B}$

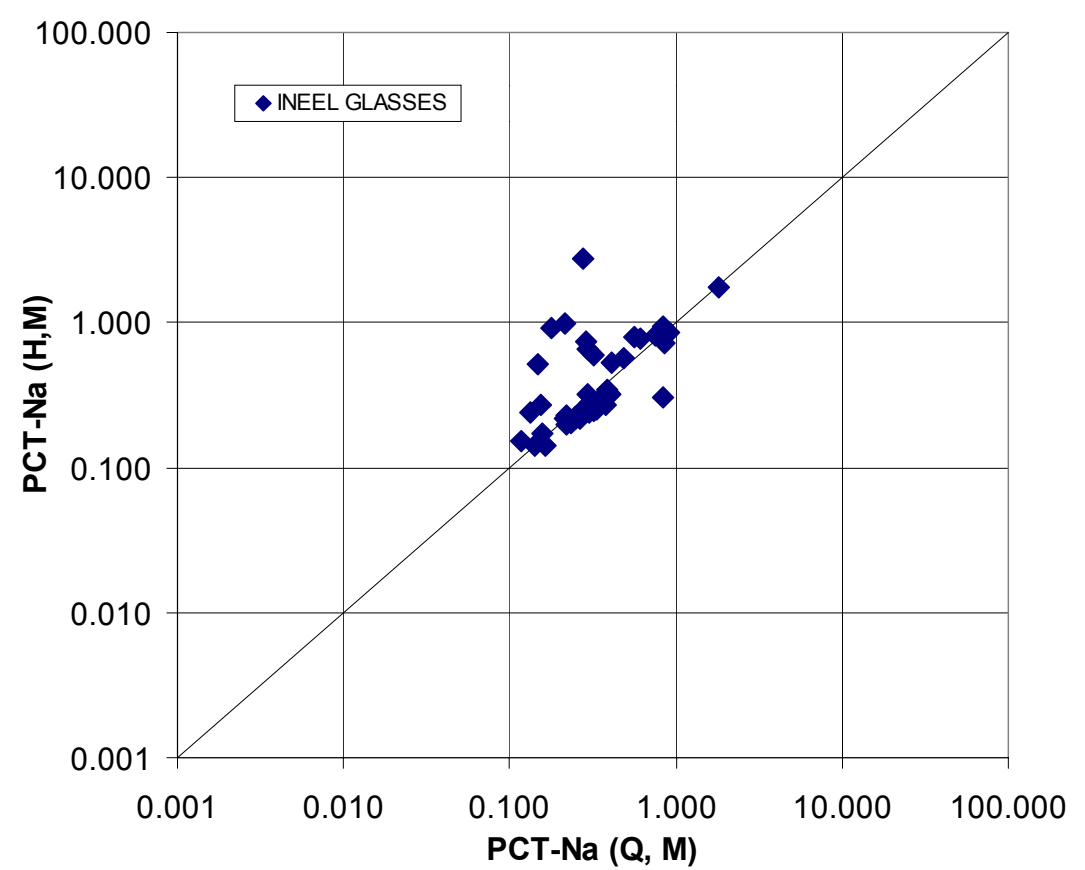

Figure A-2. INEEL Glasses: $r_{h N a}$ vs. $r_{N a}$
A.13 
PNNL-XXXXX

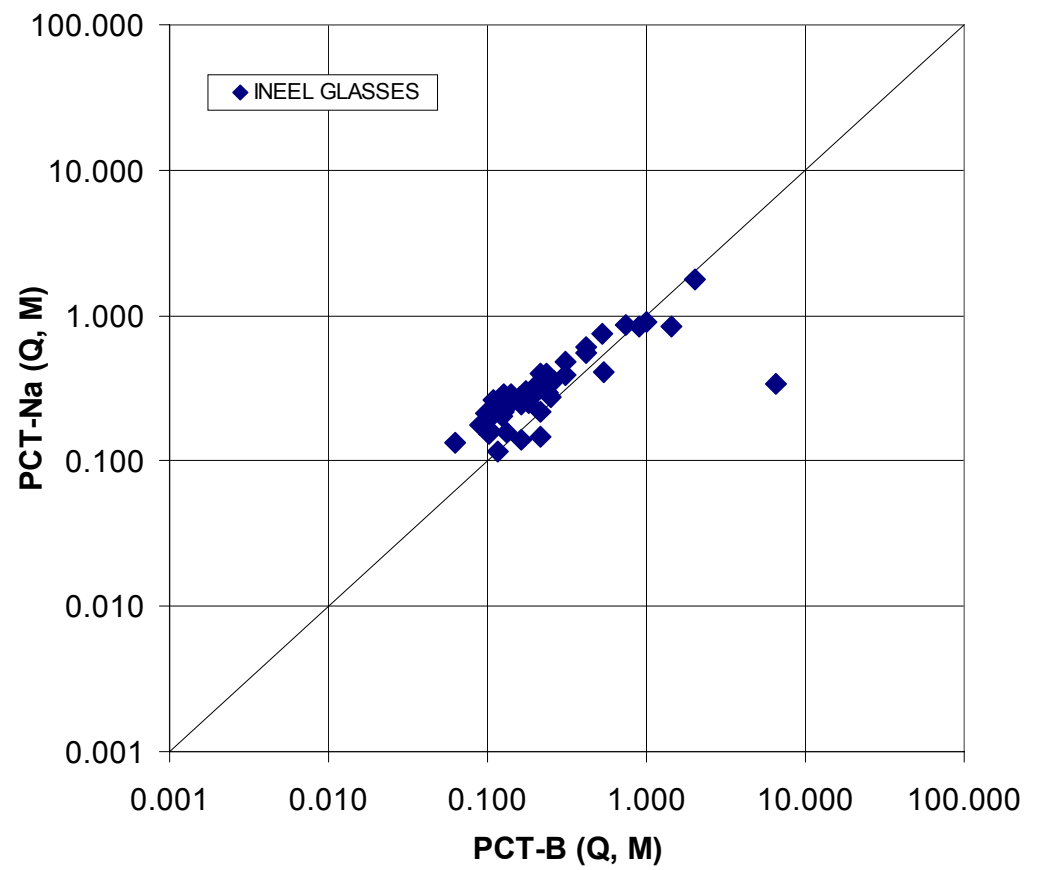

Figure A-3. INEEL Glasses: $\boldsymbol{r}_{N a}$ vs. $\boldsymbol{r}_{B}$

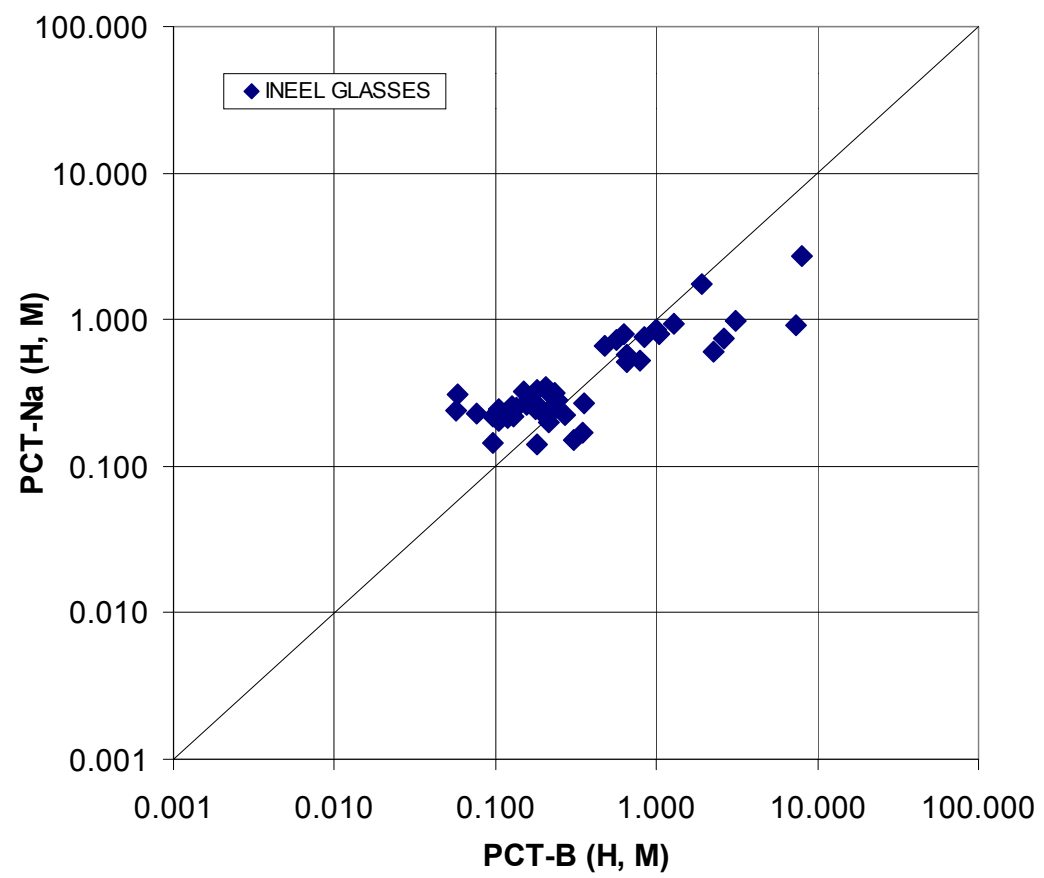

Figure A-4. INEEL Glasses: $r_{h N a}$ vs. $r_{h B}$

A. 14 
PNNL-XXXXX

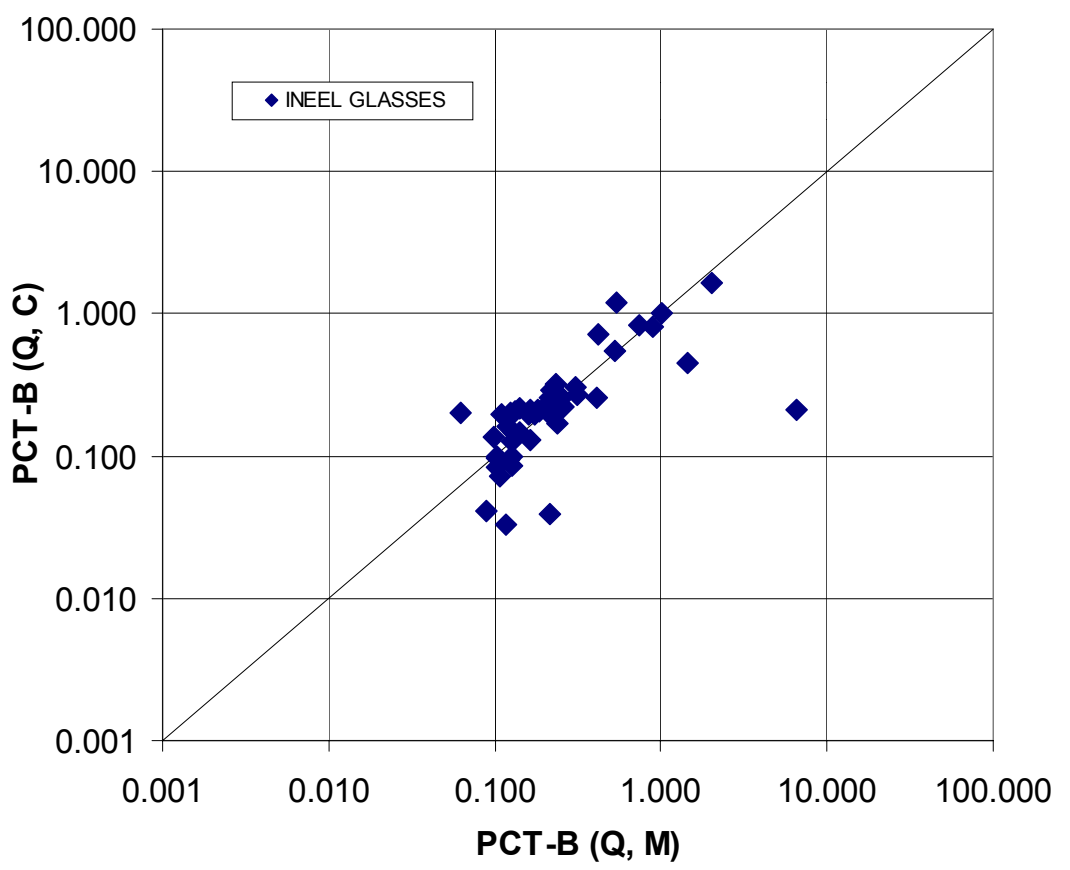

Figure A-5. INEEL Glasses: $r_{B}$, Calculated vs. Measured

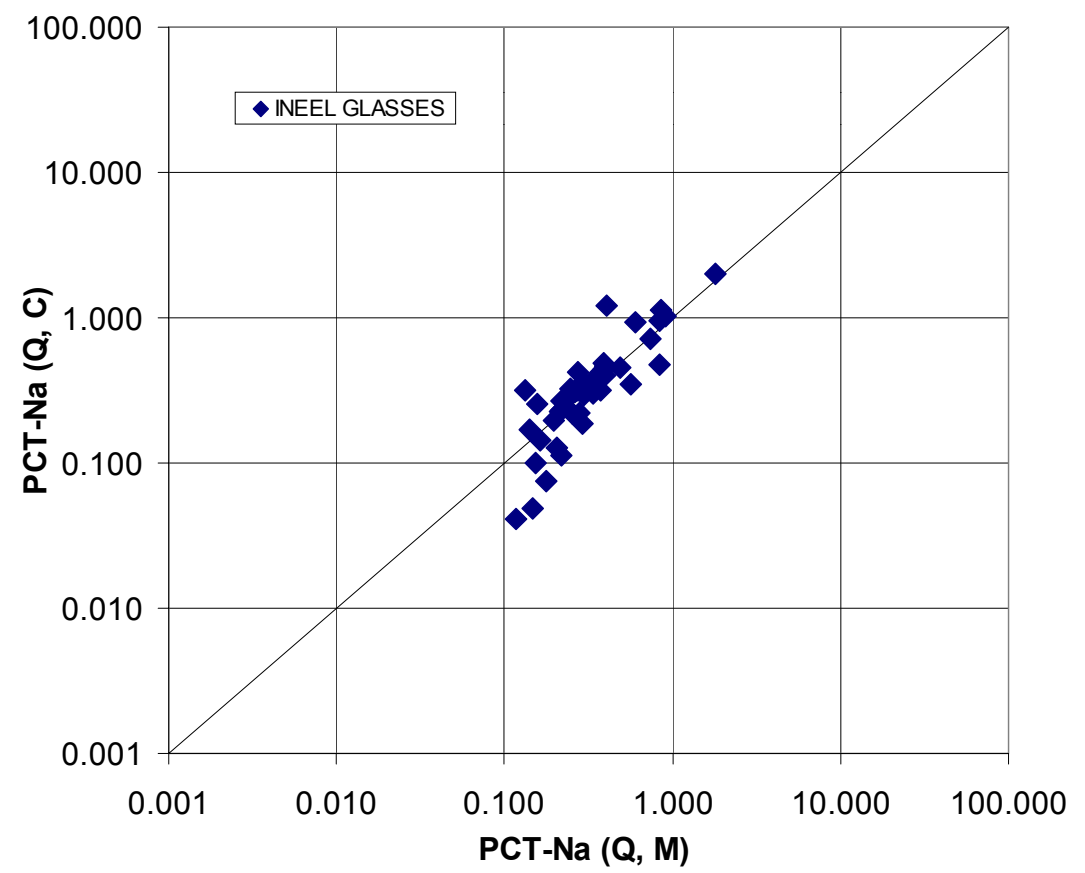

Figure A-6. INEEL Glasses: $r_{N a}$, Calculated vs. Measured

A. 15 
PNNL-XXXXX

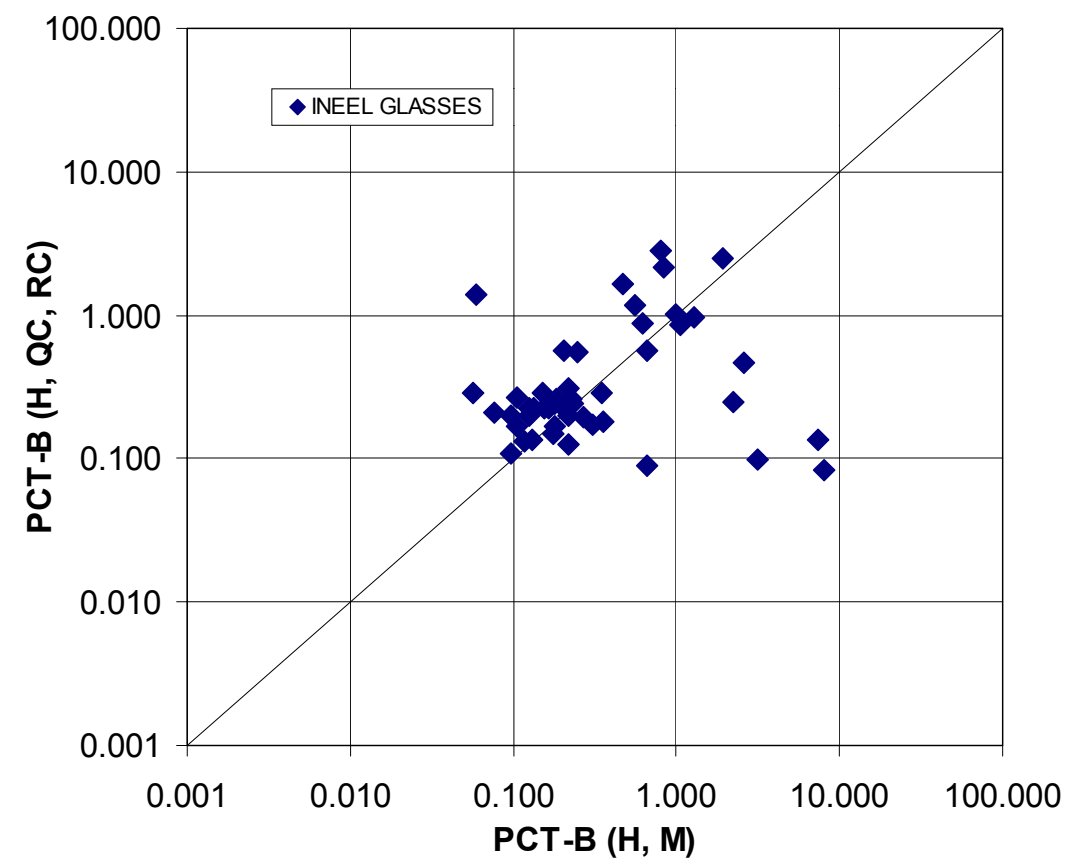

Figure A-7. INEEL Glasses: $r_{r B}$ (calculated) vs. $r_{h B}$ (measured)

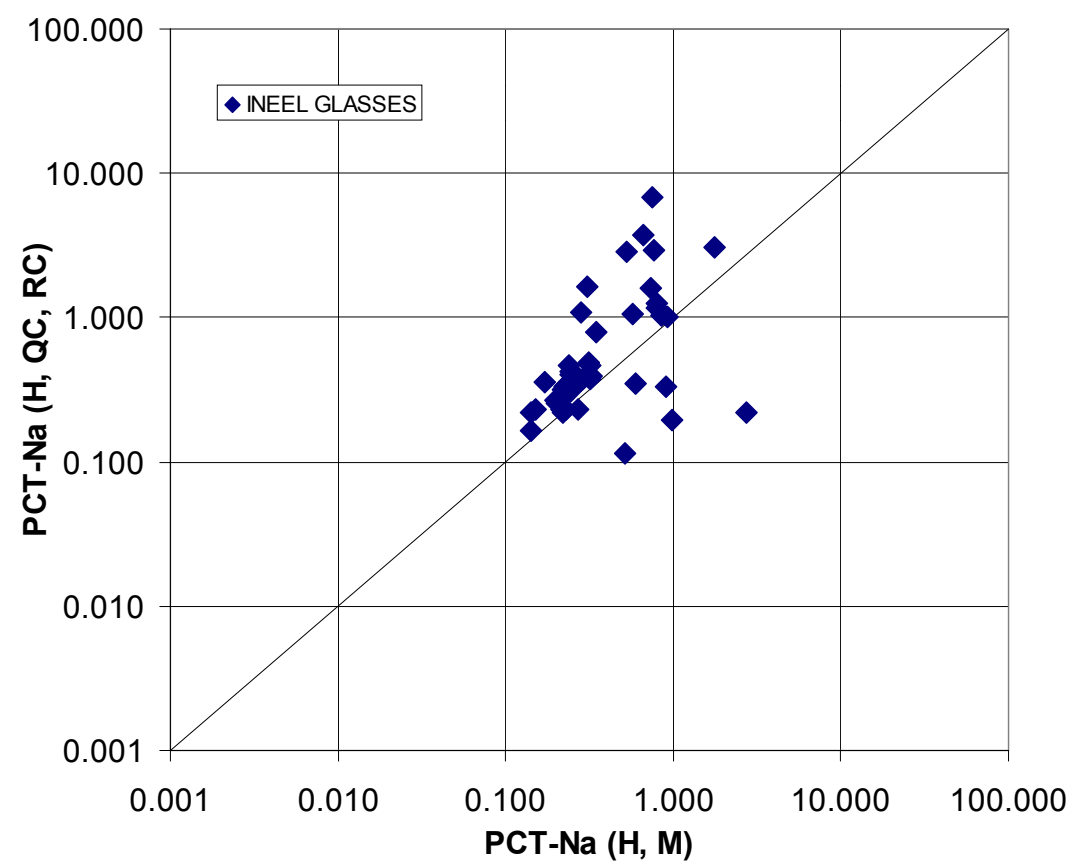

Figure A-8. INEEL Glasses: $r_{r N a}$ (calculated) vs. $r_{h N a}$ (measured)
A. 16 
PNNL-XXXXX

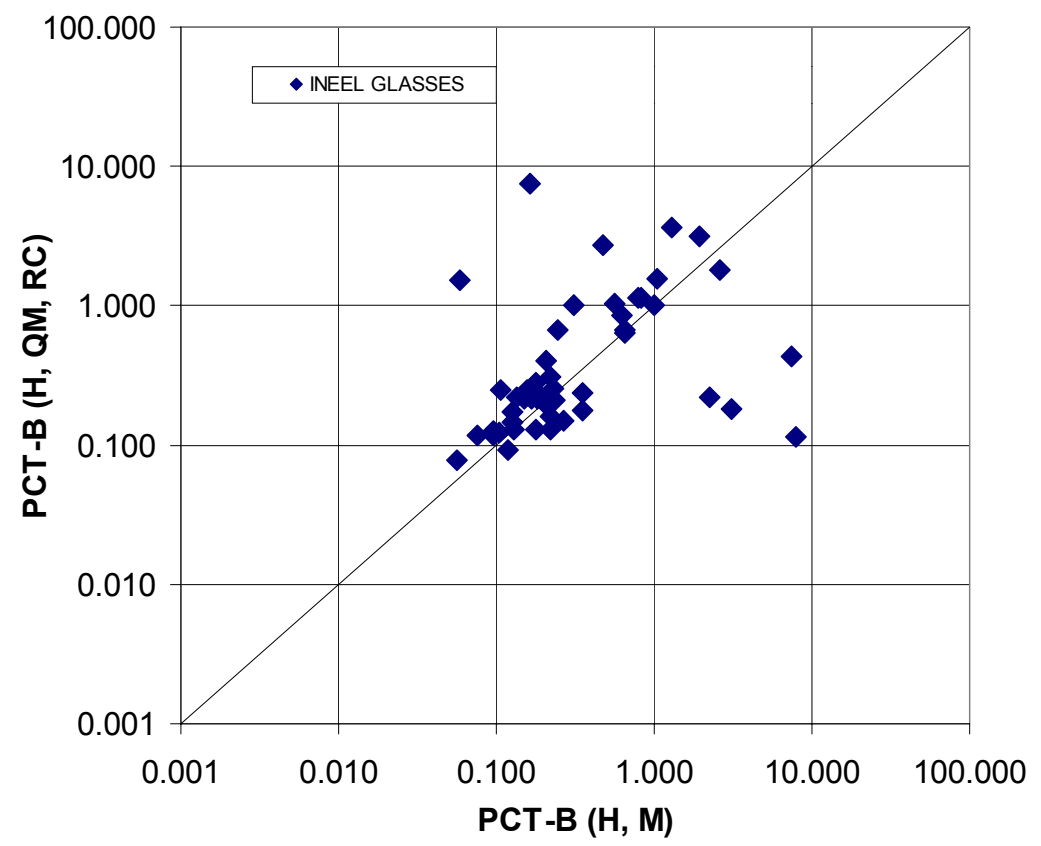

Figure A-9. INEEL Glasses: $r_{r B}$ (calculated using measured $r_{B}$ ) vs. $r_{h B}$ (measured)

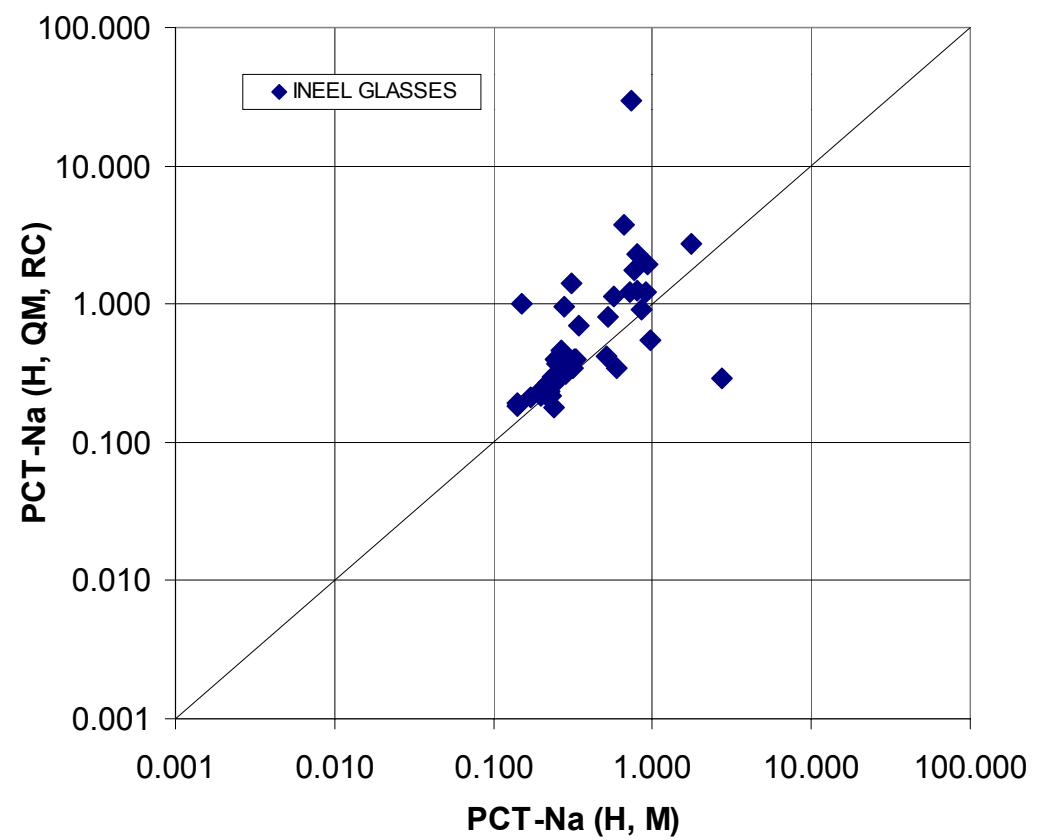

Figure A-10. INEEL Glasses: $r_{r N a}$ (calculated using measured $\left.r_{N a}\right)$ vs. $r_{h N a}$ (measured) 
PNNL-XXXXX

\section{Renormalized Releases from Heat-Treated Glasses}

The acceptability requirement is expressed in terms of the 7-day PCT, whether from quenched or CCC glass. From the theoretical point of view, it seems more appropriate to determine the $i$-th element normalized release by the formula

$$
r_{r i}=\frac{c_{i} V}{g_{r i} A_{r}}
$$

where $c_{i}$ is the $i$-th element concentration in solution, $g_{r i}$ is $i$-th element mass fraction in residual glass, $V$ is the solution volume, and $A_{r}$ is the residual glass-solution interface area. The $g_{r i}$ values can be calculated using Equation (3) and are tabulated in this report for each glass (Table 3.3, Table 3.8, Table 3.9, Table 3.20 to Table 3.23, and Table A-5). Elements that do not enter into crystalline phases accumulate in the amorphous phase. Generally, if $c_{i k}>g_{j}$, the precipitation of a mineral tends to increase $g_{j}$ in the glass (thus $g_{r i}>g_{j}$ for these elements) and if $c_{i k}<g_{j}$, then $g_{r i}<g_{j}$.

The $A_{r}$ value is difficult to assess because when the glass is crushed, the fractured surfaces may go through crystalline inclusions or around them according to the distribution of stress around inclusions. If fractures pass through inclusions, then $A_{r}<A$, where $A$ is the initial sample-solution interface area. Then it can be argued that the $g_{r i} A_{r}$ product does not significantly change as a result of crystallization for elements that do not enter into crystalline phases. For these elements, replacing $g_{r i} A_{r}$ with $g_{i} A$ in Equation (A1) would cause a negligible error in estimating $r_{r B}$ and $r_{r N a}$ by Equation (5). It is uncertain without further investigation whether $A_{r}$ is smaller than, equal to, or larger than $A$. In this report, we calculated $r_{r B}$ and $r_{r N a}$ using the formula

$$
r_{r i}=\frac{c_{i} V}{g_{r i} A}
$$

In this section, we compare $\mathrm{B}_{2} \mathrm{O}_{3}$ and $\mathrm{Na}_{2} \mathrm{O}$ mass fractions in the original and residual glasses for each major group of compositions (glasses precipitating clinopyroxene, NP, other phases, and INEEL glasses). Then we normalized the B and Na PCT releases from heat-treated glasses using calculated residual glass compositions. Finally, we compared $r_{h N a}$ and $r_{h B}$ based on the residual and original glass compositions.

Compositions of original (quenched) glasses are in Table 3.1, Table 3.6, Table 3.12 to Table 3.15, and Table A-2. Compositions of residual glasses are in Table 3.3, Table 3.8 and Table 3.9, Table 3.20 to Table 3.23, and Table A-5. Mass fractions of $\mathrm{B}_{2} \mathrm{O}_{3}$ and $\mathrm{Na}_{2} \mathrm{O}$ in residual and original glasses are compared in Figures A-11 to A-18. The $\mathrm{B}_{2} \mathrm{O}_{3}$ content is always increased in the residual glass because $\mathrm{B}_{2} \mathrm{O}_{3}$ does not participate in the formation of any crystalline phase listed in Table 2.3 and Table A-3. The 
excess of $\mathrm{B}_{2} \mathrm{O}_{3}$ in residual glass over that in the corresponding original glass is proportional to the fraction of total crystallinity in the heat-treated glass - see Equation (3).

Unlike $\mathrm{B}_{2} \mathrm{O}_{3}, \mathrm{Na}_{2} \mathrm{O}$ participates in some crystalline phases, such as clinopyroxene and NP. If the $\mathrm{Na}_{2} \mathrm{O}$ fraction is higher or lower in the mineral than in the glass, then the $\mathrm{Na}_{2} \mathrm{O}$ content will decrease or increase as the mineral precipitates. If the $\mathrm{Na}_{2} \mathrm{O}$ fraction is approximately the same in the mineral as in the glass, mineral precipitation has little effect on the $\mathrm{Na}_{2} \mathrm{O}$ content in the amorphous phase. This is the case of glasses precipitating clinopyroxene, as seen in Figure A-15. In the case of glasses precipitating $\mathrm{NP}$, residual glass is somewhat impoverished of $\mathrm{Na}_{2} \mathrm{O}$ with the exception of four compositions, as seen in Figure A-16. Figure A-17 shows a slight increase in $\mathrm{Na}_{2} \mathrm{O}$ in glasses precipitating major phases other than clinopyroxene and NP. As Table 2.3 shows, mineral phases precipitating from these glasses have zero $\mathrm{Na}_{2} \mathrm{O}$ content. The $\mathrm{Na}_{2} \mathrm{O}$ content in minerals that precipitated in INEEL glasses was either zero or comparable to that of glass (Table A-3). As a result, some residual glasses had excess and others lack of $\mathrm{Na}_{2} \mathrm{O}$ as compared to their quenched counterparts (Figure A-18).

Releases renormalized with respect to residual glass are listed in Tables A-8 to A-10. As Figures A19 to A-24 show, renormalization had little effect on $r_{h N a}$ and $r_{h B}$ values. Exceptions are only releases from glasses with massive precipitation of crystals. These glasses are extreme and unsuitable for HLW vitrification. Releases of $\mathrm{Si}, \mathrm{Al}$, and other glass-forming elements common in silicate minerals may be more affected by the way of normalization. However, these elements are even more affected by precipitation of minerals in the solution. 
PNNL-XXXXX

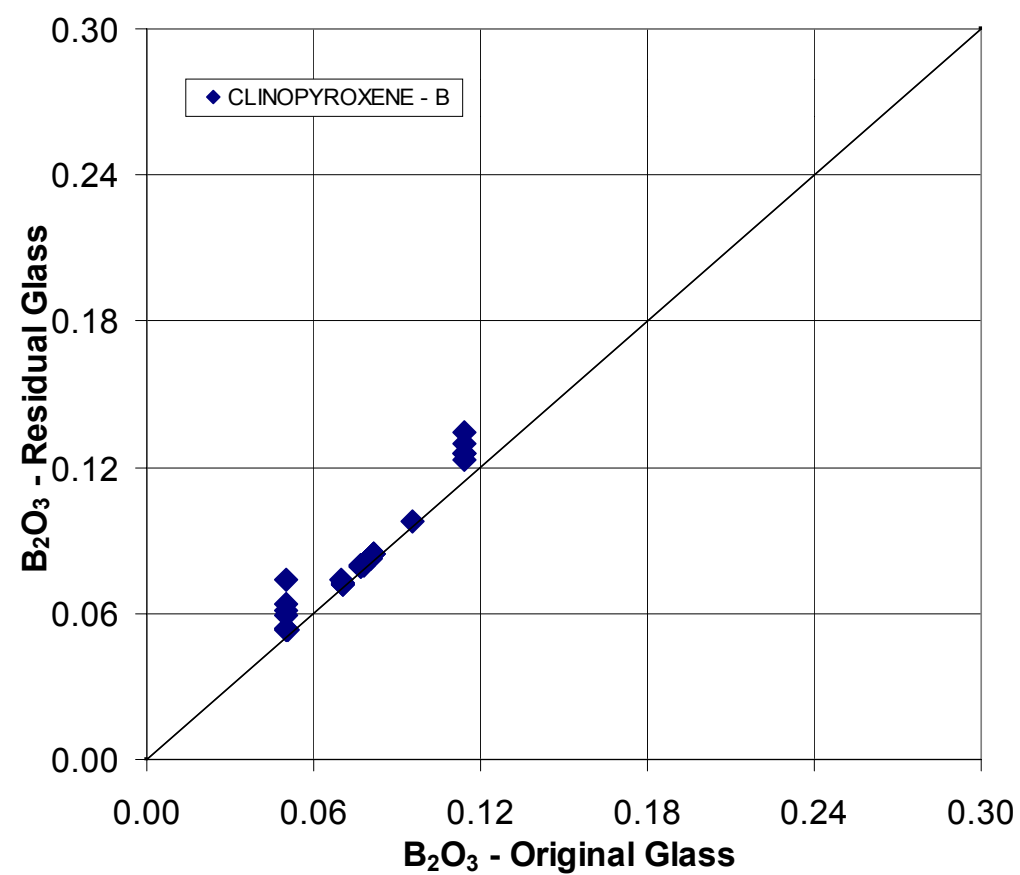

Figure A-11. Mass Fraction of $\mathrm{B}_{2} \mathrm{O}_{3}$ in Glasses Precipitating Clinopyroxene, $g_{r B}$ vs. $g_{B}$

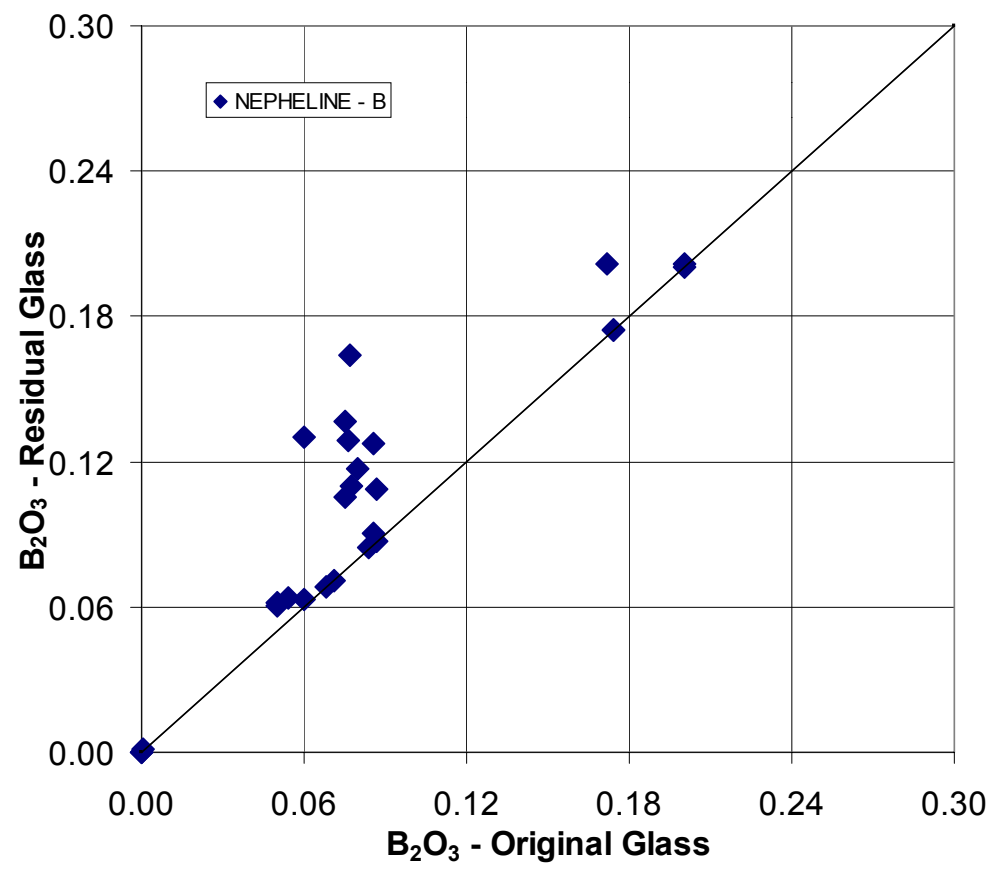

Figure A-12. Mass Fraction of $\mathrm{B}_{2} \mathrm{O}_{3}$ in Glasses Precipitating NP, $g_{r b}$ vs. $g_{B}$ 
PNNL-XXXXX

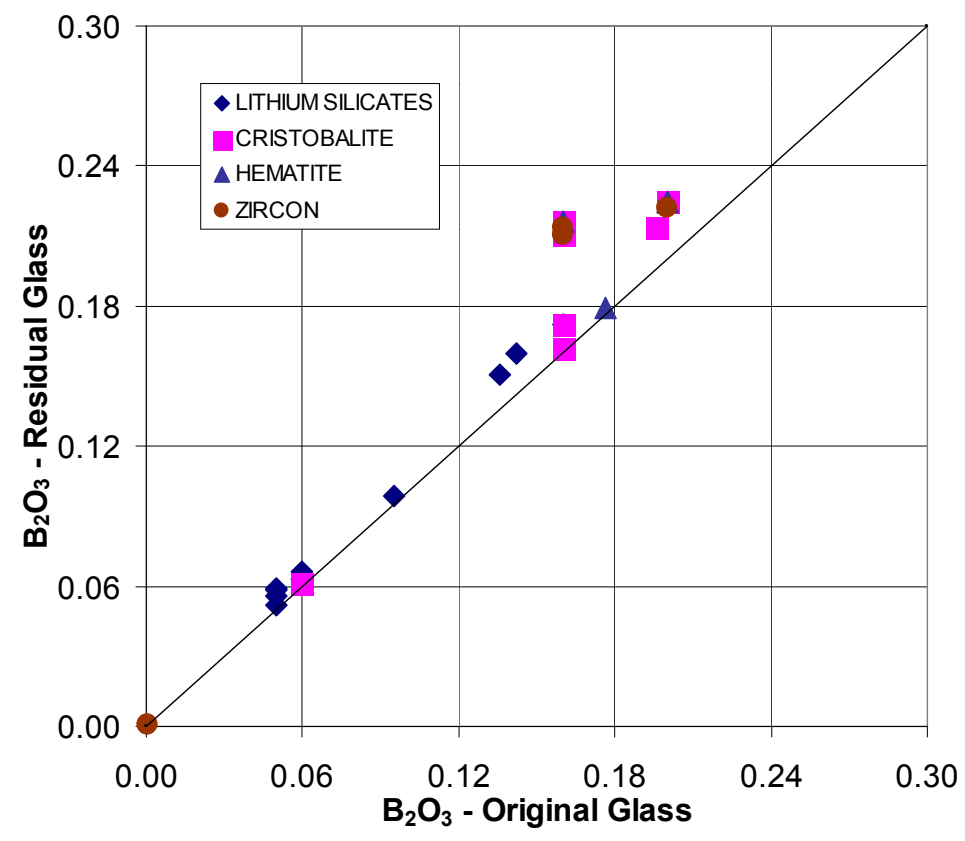

Figure A-13. Mass Fraction of $\mathrm{B}_{2} \mathrm{O}_{3}$ in Glasses Precipitating Phases other than Clinopyroxene and NP, $g_{r B}$ vs. $g_{B}$

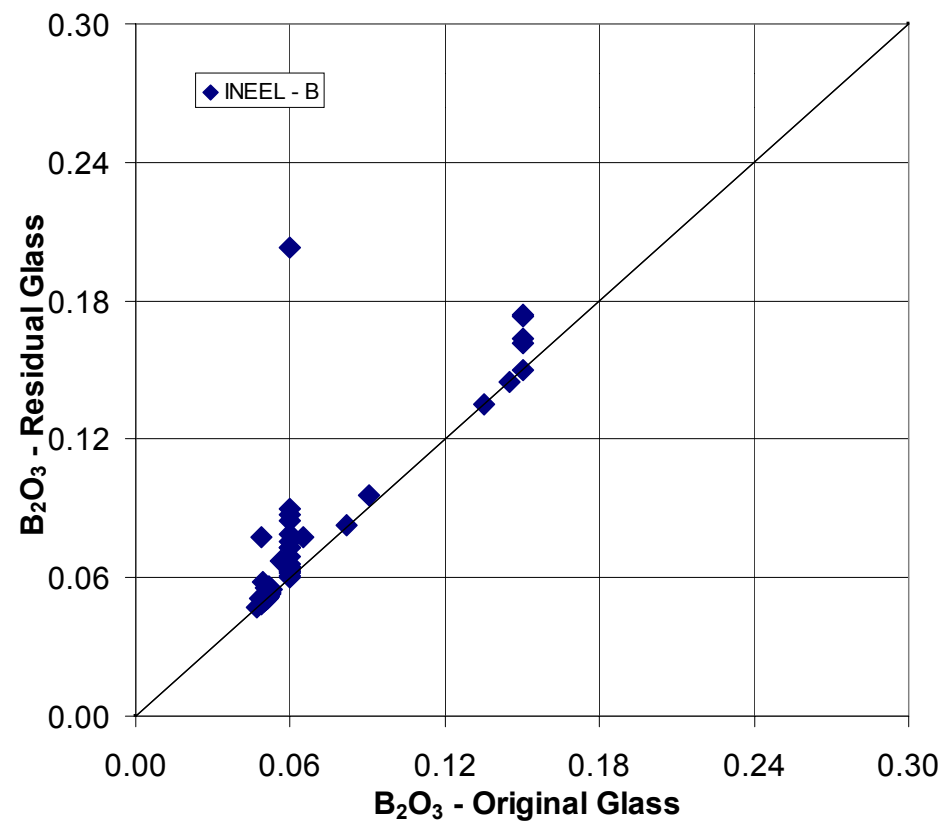

Figure A-14. Mass Fraction of $\mathrm{B}_{2} \mathrm{O}_{3}$ in INEEL Glasses, $g_{r B}$ vs. $g_{B}$ 
PNNL-XXXXX

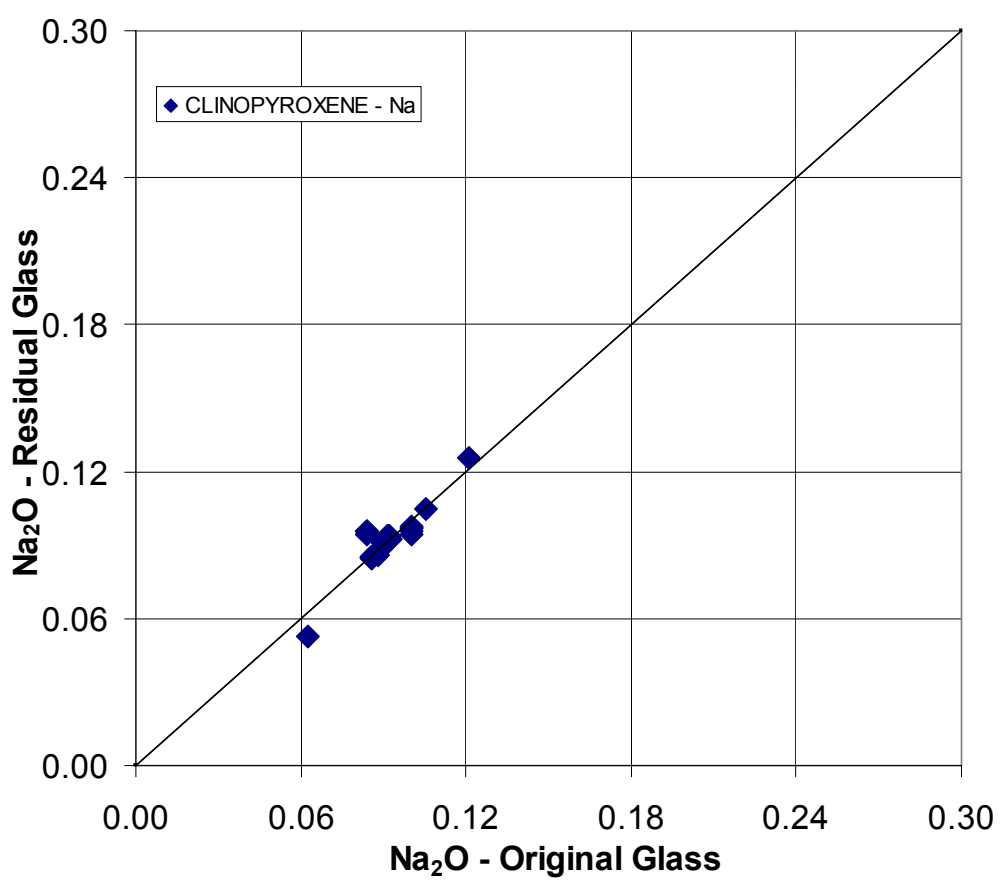

Figure A-15. Mass Fraction of $\mathrm{Na}_{2} \mathrm{O}$ Precipitating Clinopyroxene, $g_{r N a}$ vs. $g_{N a}$

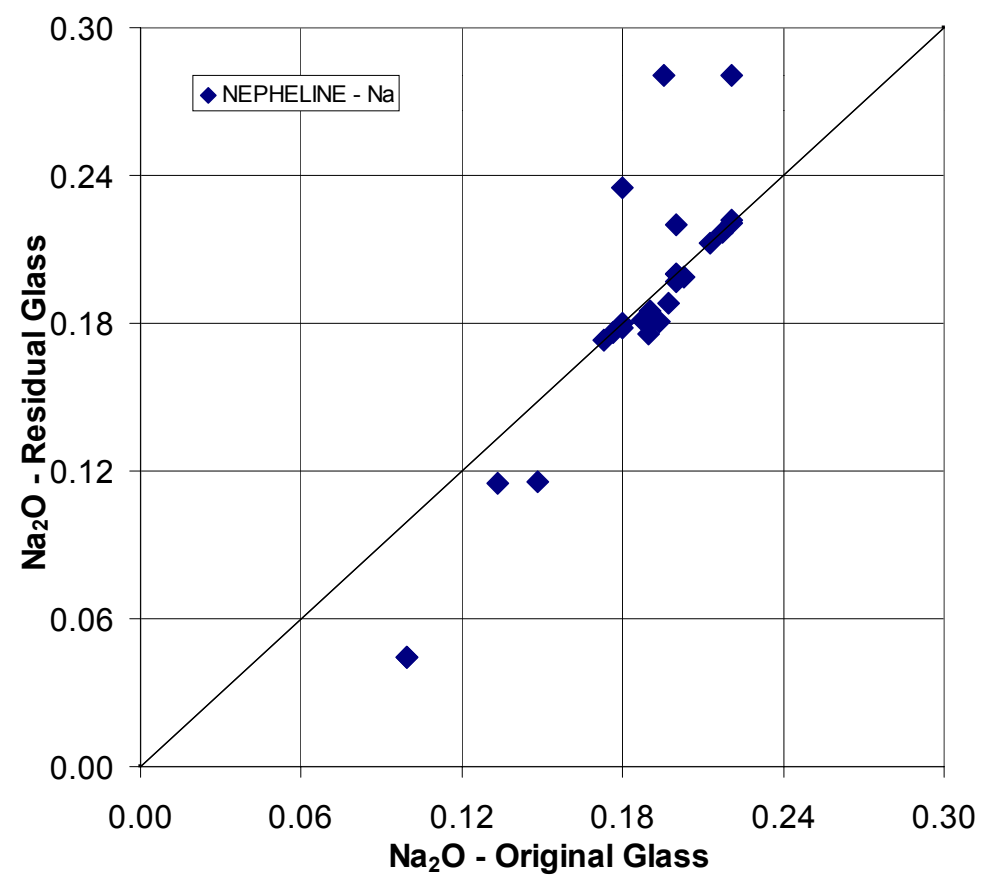

Figure A-16. Mass Fraction of $\mathrm{Na}_{2} \mathrm{O}$ Precipitating NP, $g_{r N a}$ vs. $g_{\mathrm{Na}}$ 
PNNL-XXXXX

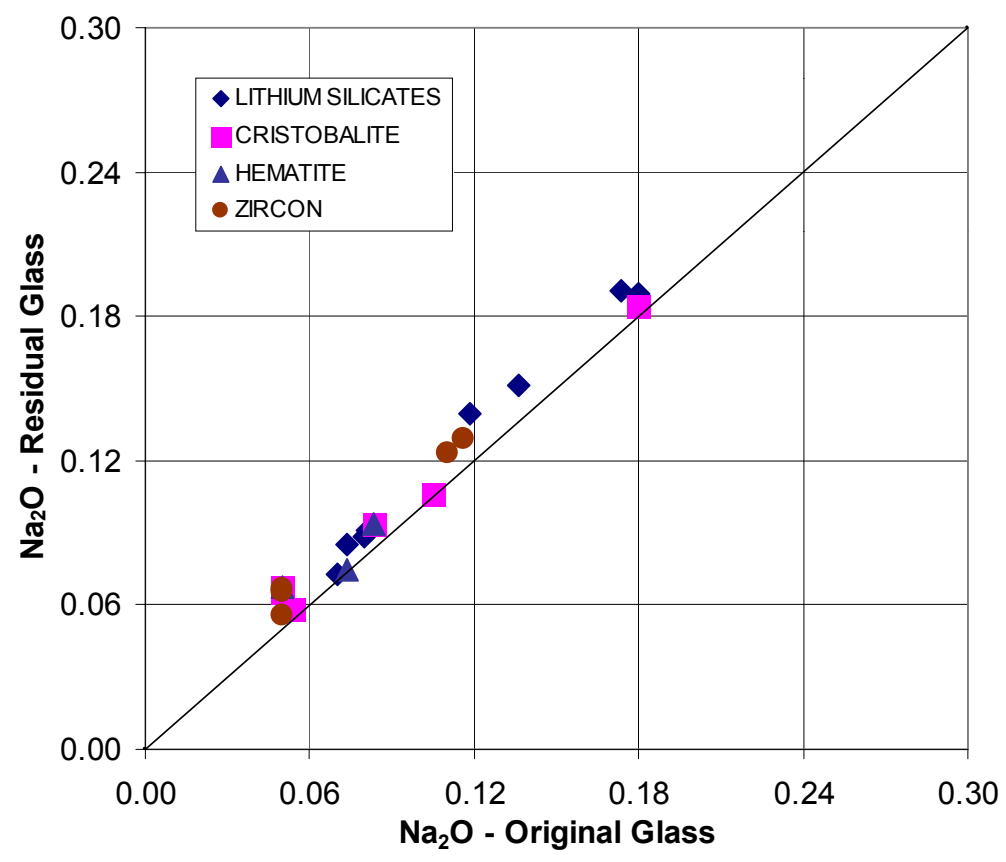

Figure A-17. Mass Fraction of $\mathrm{Na}_{2} \mathrm{O}$ in Glasses Precipitating Phases other than Clinopyroxene and NP, $g_{r N a}$ vs. $g_{N a}$

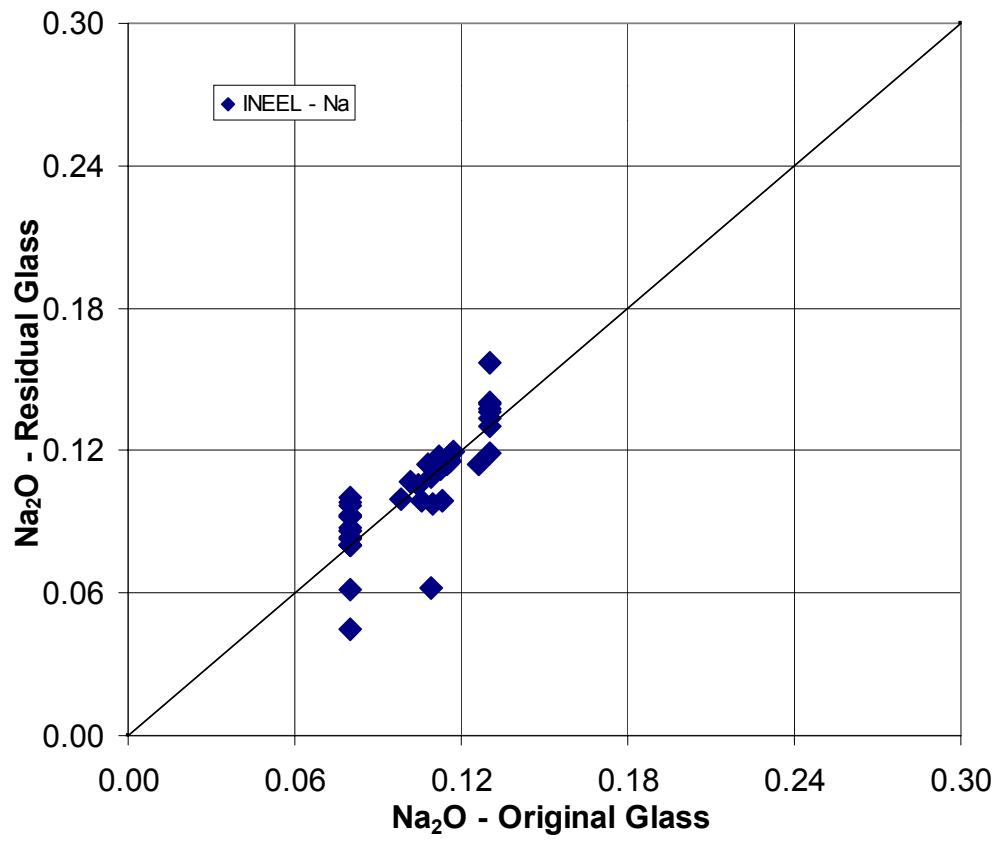

Figure A-18. Mass Fraction of $\mathrm{Na}_{2} \mathrm{O}$ in INEEL Glasses, $g_{r N a}$ vs. $g_{N a}$ 
PNNL-XXXXX

Table A-8. Glasses Precipitating Clinopyroxene: $r_{r B}$ and $r_{r N a}$ (in $\mathrm{g} / \mathrm{m}^{2}$ ) Normalized with Respect to Residual Glass

\begin{tabular}{|l|c|c|}
\hline Glass & $\mathbf{H}, \mathbf{M}$ & $\mathbf{H}, \mathbf{M}$ \\
\hline & $\boldsymbol{r}_{\boldsymbol{r} \boldsymbol{B}}$ & $\boldsymbol{r}_{\boldsymbol{r} \mathbf{N}}$ \\
\hline CVS1-1 & 0.34 & 0.37 \\
\hline CVS1-8 & 3.43 & 1.45 \\
\hline CVS1-15 & 18.53 & 13.55 \\
\hline CVS1-19 & 0.37 & 0.37 \\
\hline CVS1-20 & 0.40 & 0.37 \\
\hline CVS1-22 & 3.24 & 1.35 \\
\hline CVS1-23 & 0.36 & 0.34 \\
\hline CVS2-2 & 0.13 & 0.16 \\
\hline CVS2-25 & 1.15 & 0.92 \\
\hline CVS2-36 & 0.33 & 0.43 \\
\hline CVS2-42 & 4.12 & 4.45 \\
\hline CVS2-50 & 0.50 & 0.39 \\
\hline CVS2-71 & 0.36 & 0.32 \\
\hline CVS2-96 & 0.56 & 0.37 \\
\hline Blend1171 & 0.35 & 0.33 \\
\hline Blend1172 & 0.35 & 0.32 \\
\hline Blend1272 & 0.38 & 0.32 \\
\hline Batch 1171 & 0.33 & 0.34 \\
\hline Batch 1172 & 0.29 & 0.30 \\
\hline Batch 1271 & 0.40 & 0.37 \\
\hline Batch 1272 & 0.32 & 0.32 \\
\hline Batch 2171 & 0.30 & 0.28 \\
\hline Batch 2172 & 0.29 & 0.29 \\
\hline Batch 2271 & 0.34 & 0.30 \\
\hline Batch 2272 & 0.34 & 0.30 \\
\hline Batch 3171 & 0.42 & 0.39 \\
\hline Batch 3172 & 0.43 & 0.39 \\
\hline Batch 3271 & 0.38 & 0.35 \\
\hline Batch 3272 & 0.39 & 0.36 \\
\hline Batch 4171 & 0.40 & 0.38 \\
\hline Batch 4172 & 0.44 & 0.43 \\
\hline Batch 4272 & 0.45 & 0.43 \\
\hline HM171 & 0.28 & 0.24 \\
\hline HM172 & 0.25 & 0.21 \\
\hline HM271 & 0.28 & 0.24 \\
\hline HM272 & 0.28 & 0.23 \\
\hline \hline
\end{tabular}


PNNL-XXXXX

Table A-9. Glasses Precipitating NP: $r_{r B}$ and $r_{r N a}\left(\right.$ in $\left.\mathrm{g} / \mathrm{m}^{2}\right)$ Normalized with Respect to Residual Glass

\begin{tabular}{|l|r|r|}
\hline Glass & \multicolumn{1}{|c|}{$\mathbf{H}, \mathbf{M}$} & \multicolumn{1}{|c|}{, $\mathbf{M}$} \\
\hline & \multicolumn{1}{c|}{$\boldsymbol{r}_{\boldsymbol{r} \boldsymbol{B}}$} & \multicolumn{1}{c|}{$\boldsymbol{r}_{\boldsymbol{r} \boldsymbol{} \boldsymbol{a}}$} \\
\hline CVS1-16 & 18.949 & 9.159 \\
\hline CVS2-33 & 9.801 & 7.530 \\
\hline CVS2-34 & 4.520 & 2.590 \\
\hline CVS2-35 & 18.776 & 9.437 \\
\hline CVS2-63 & 3.103 & 2.218 \\
\hline CVS2-85 & 20.953 & 14.059 \\
\hline CVS2-86 & 0.257 & 0.303 \\
\hline CVS2-98 & 2.970 & 2.760 \\
\hline CVS3-16 & 0.338 & 0.089 \\
\hline NP-BL & 29.325 & 21.282 \\
\hline NP-A1-1 & 1.730 & 1.870 \\
\hline NP-A1-2 & 8.645 & 6.592 \\
\hline NP-A1-3 & 27.237 & 18.407 \\
\hline NP-A1-4 & 24.627 & 16.651 \\
\hline NP-Na-1 & 1.282 & 0.462 \\
\hline NP-Na-2 & 14.811 & 7.646 \\
\hline NP-Si-3 & 0.360 & 0.490 \\
\hline NP-Si-4 & 0.280 & 0.410 \\
\hline NP-B-1 & - & 1.164 \\
\hline NP-B-2 & - & - \\
\hline NP-K-1 & 34.070 & 28.316 \\
\hline NP-K-2 & 32.378 & 29.687 \\
\hline NP-Li-1 & 0.752 & 0.590 \\
\hline NP-Li-2 & 0.838 & 1.074 \\
\hline \hline
\end{tabular}


PNNL-XXXXX

Table A-10. INEEL Glasses: $r_{r B}$ and $r_{r N a}\left(\right.$ in $\left.g / \mathrm{m}^{2}\right)$ Normalized with Respect to Residual Glass

\begin{tabular}{||l|c|c||}
\hline Glass & $\mathbf{H , ~ M}$ & $\mathbf{H , ~}$ \\
\hline & $\mathbf{r}_{\mathbf{r B}}$ & $\mathbf{r}_{\mathbf{r N a}}$ \\
\hline DZr-CV-1 & 0.218 & 0.311 \\
\hline DZr-CV-2 & 1.964 & 1.710 \\
\hline DZr-CV-3 & 0.164 & 0.280 \\
\hline DZr-CV-4 & 0.054 & 0.294 \\
\hline DZr-CV-5 & 2.204 & 0.590 \\
\hline DZr-CV-6 & 0.177 & 0.242 \\
\hline DZr-CV-7 & 0.125 & 0.237 \\
\hline DZr-CV-8 & 0.224 & 0.306 \\
\hline DZr-CV-9 & 0.160 & 0.280 \\
\hline DZr-CV-10 & 0.151 & 0.255 \\
\hline DZr-CV-11 & 0.132 & 0.247 \\
\hline DZr-CV-12 & 0.100 & 0.230 \\
\hline DZr-CV-13 & 0.109 & 0.213 \\
\hline DZr-CV-14 & 0.182 & 0.327 \\
\hline DZr-CV-15 & 0.092 & 0.137 \\
\hline DZr-CV-16 & 0.151 & 0.322 \\
\hline DZr-CV-17 & 0.076 & 0.225 \\
\hline DZr-CV-18 & 0.169 & 0.256 \\
\hline DZr-CV-19 & 0.096 & 0.219 \\
\hline DZr-CV-20 & 0.052 & 0.273 \\
\hline DZr-CV-21 & 0.555 & 0.635 \\
\hline DZr-CV-22 & 0.103 & 0.201 \\
\hline DZr-CV-23 & 0.125 & 0.252 \\
\hline DZr-CV-24 & 0.110 & 0.245 \\
\hline DP-Centroid & 0.198 & 0.220 \\
\hline DP-BL1 & 0.253 & 0.240 \\
\hline DP-BL2 & 0.219 & 0.245 \\
\hline DP-1 & 4.928 & 1.619 \\
\hline DP-2 & 0.768 & - \\
\hline DP-3 & 0.271 & 0.350 \\
\hline DP-4 & 0.573 & 0.446 \\
\hline DP-5 & 0.218 & 0.123 \\
\hline DP-6 & 0.829 & 0.666 \\
\hline DP-7 & 0.214 & 0.199 \\
\hline DP-8 & 1.115 & 0.804 \\
\hline DP-9 & 1.784 & 1.639 \\
\hline DP-10 & 0.687 & 0.452 \\
\hline DP-11 & 0.992 & 0.856 \\
\hline DP-12 & 0.218 & 0.228 \\
\hline
\end{tabular}

A.26 
PNNL-XXXXX

Table A-10 (Contd)

\begin{tabular}{|l|c|c||}
\hline Glass & $\mathbf{H}, \mathbf{M}$ & $\mathbf{H}, \mathbf{M}$ \\
\hline & $\mathbf{r}_{\mathbf{r B}}$ & $\mathbf{r}_{\mathbf{r N a}}$ \\
\hline DP-13 & 0.326 & 0.527 \\
\hline DP-14 & 0.202 & 0.232 \\
\hline DP-15 & - & 0.183 \\
\hline DP-16 & 0.168 & 0.131 \\
\hline DP-17 & 0.520 & 0.679 \\
\hline DP-18 & 0.195 & 0.327 \\
\hline DP-19 & 0.593 & 0.769 \\
\hline DP-20 & - & 0.154 \\
\hline DP-21 & 0.229 & 0.243 \\
\hline DP-22 & 0.698 & 0.746 \\
\hline DP-23 & 6.521 & 2.984 \\
\hline DP-24 & 0.320 & 0.155 \\
\hline \hline
\end{tabular}


PNNL-XXXXX

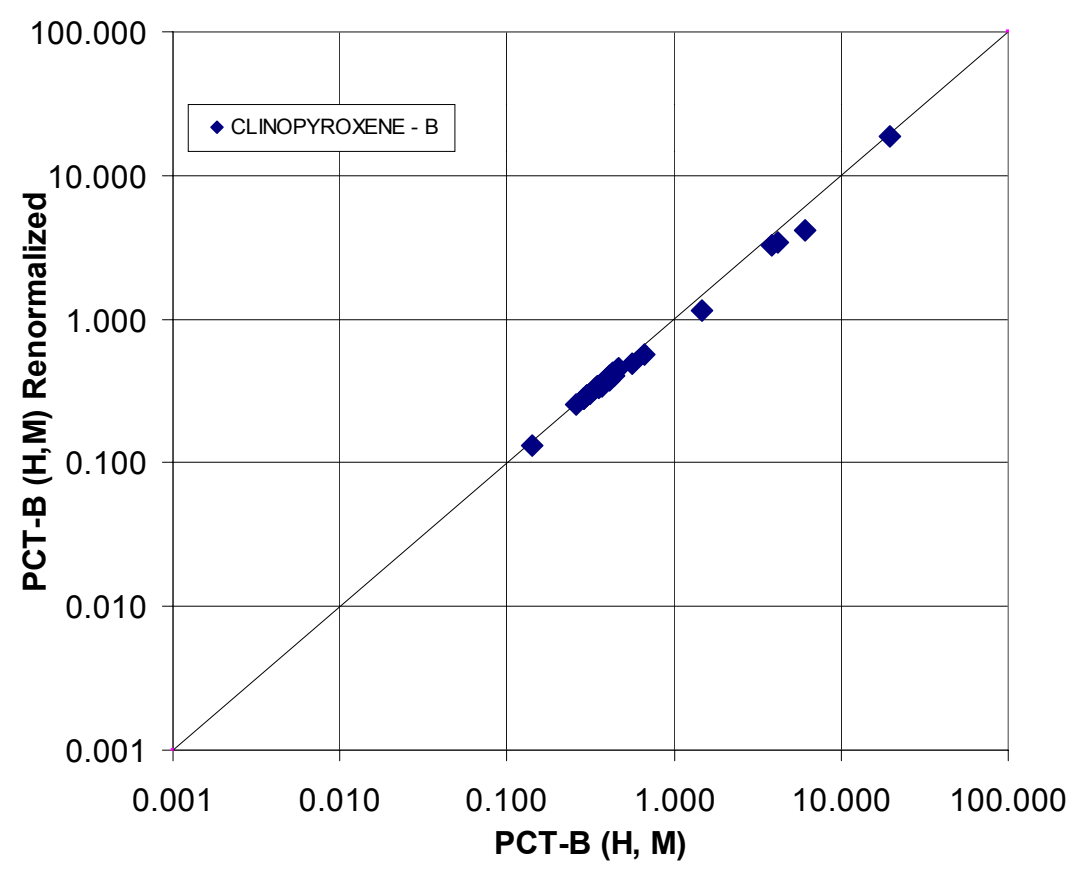

Figure A-19. Glasses Precipitating Clinopyroxene: $r_{h b}$, Residual vs. Original

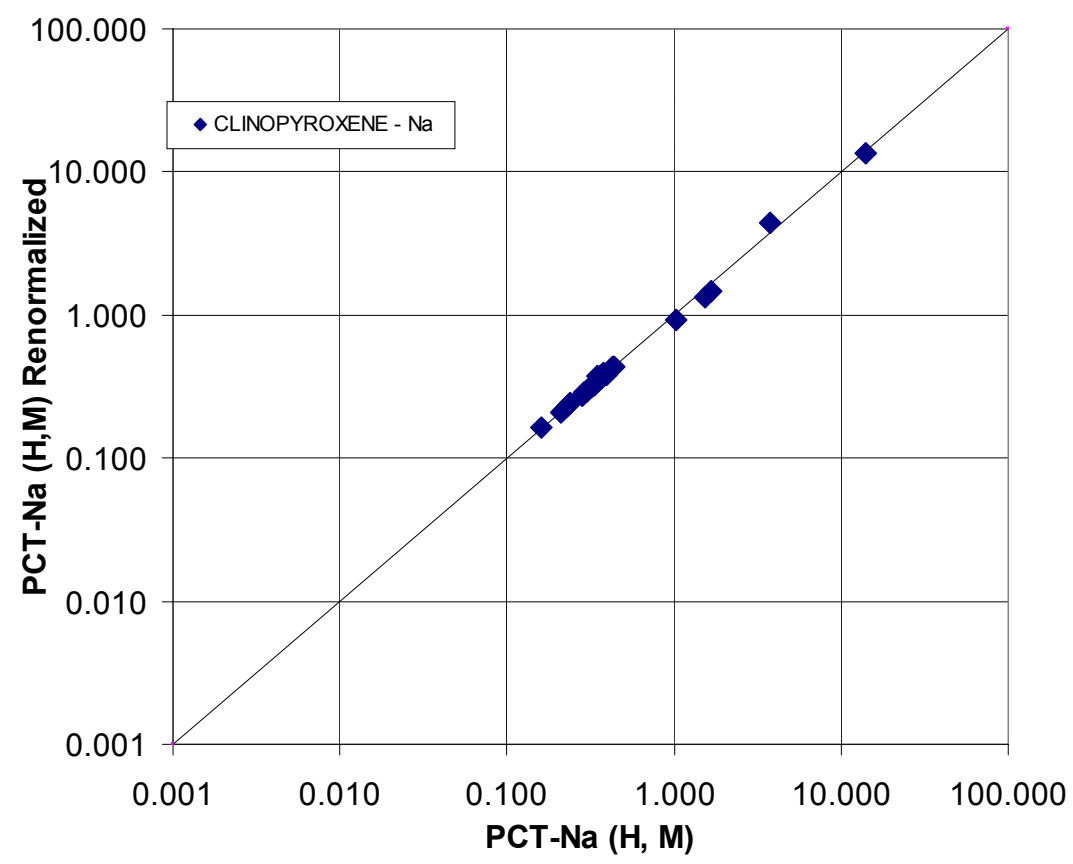

Figure A-20. Glasses Precipitating Clinopyroxene: $r_{h N a}$, Residual vs. Original A. 28 
PNNL-XXXXX

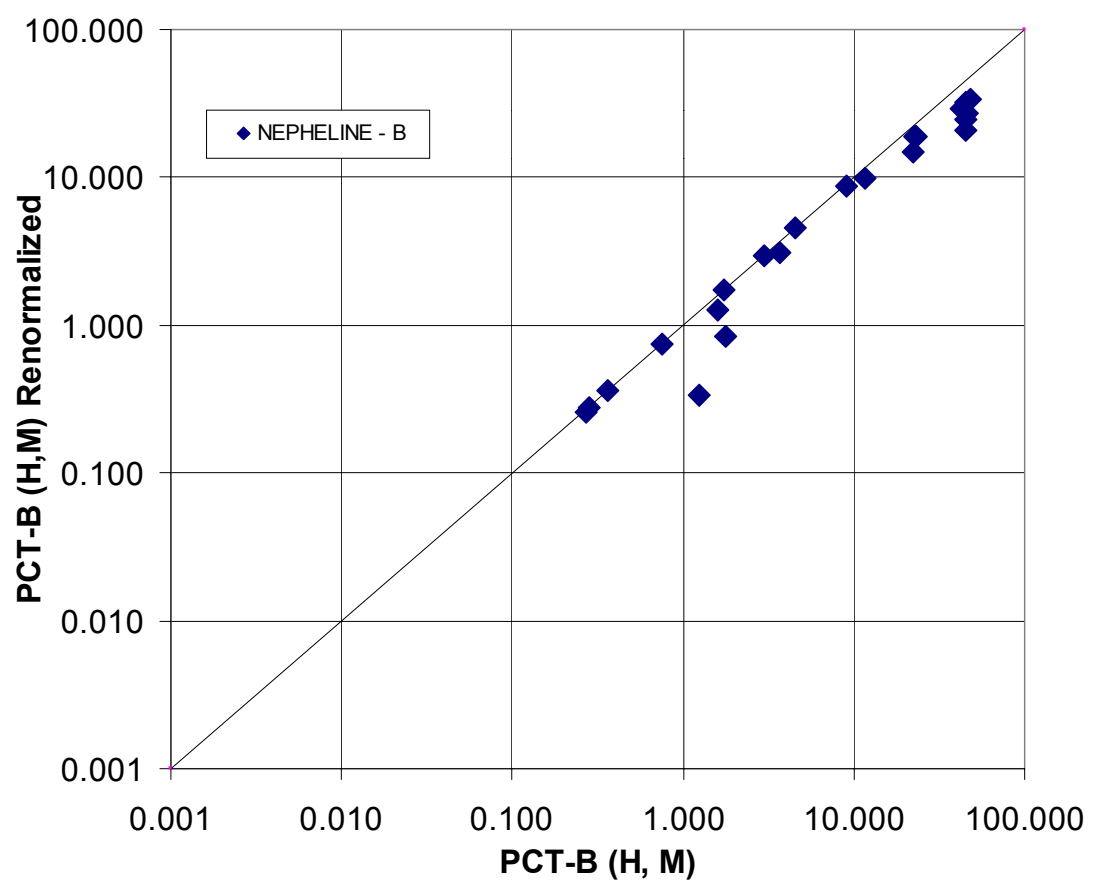

Figure A-21. Glasses Precipitating NP: $r_{h B}$, Residual vs. Original

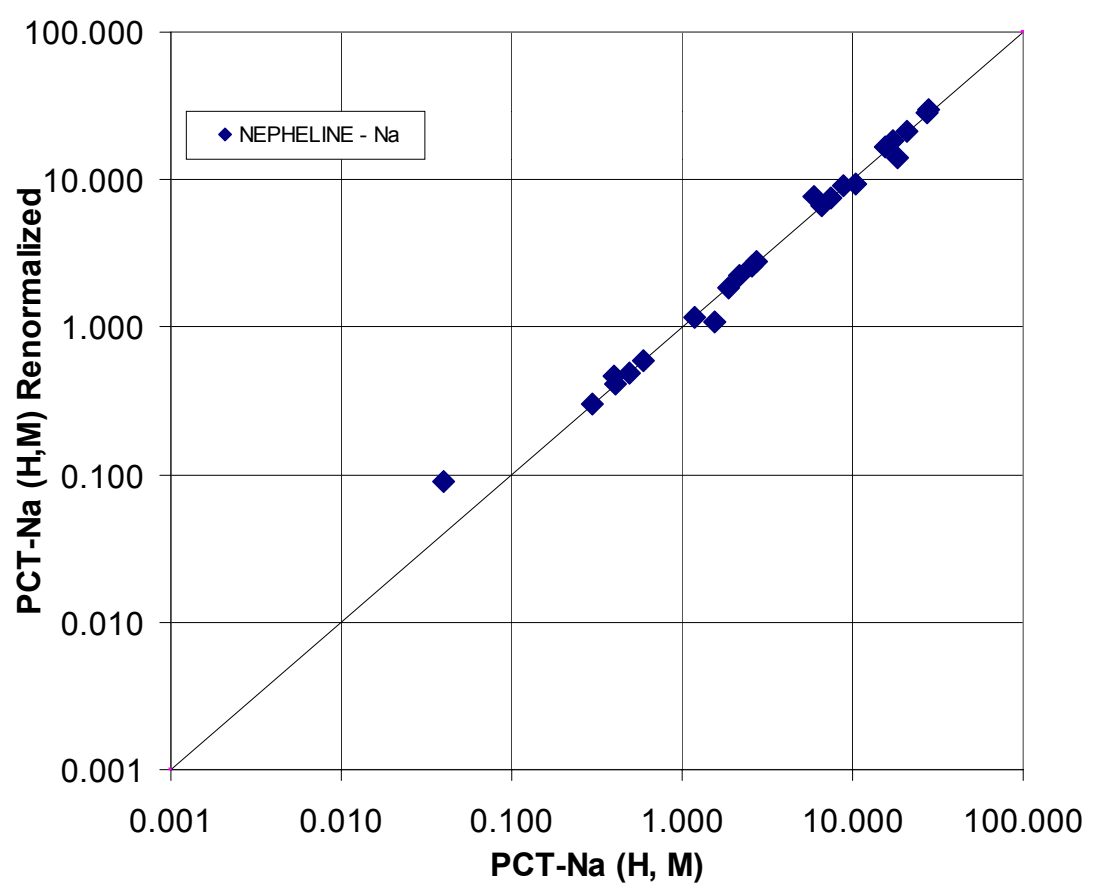

Figure A-22. Glasses Precipitating NP: $r_{h N a}$, Residual vs. Original 
PNNL-XXXXX

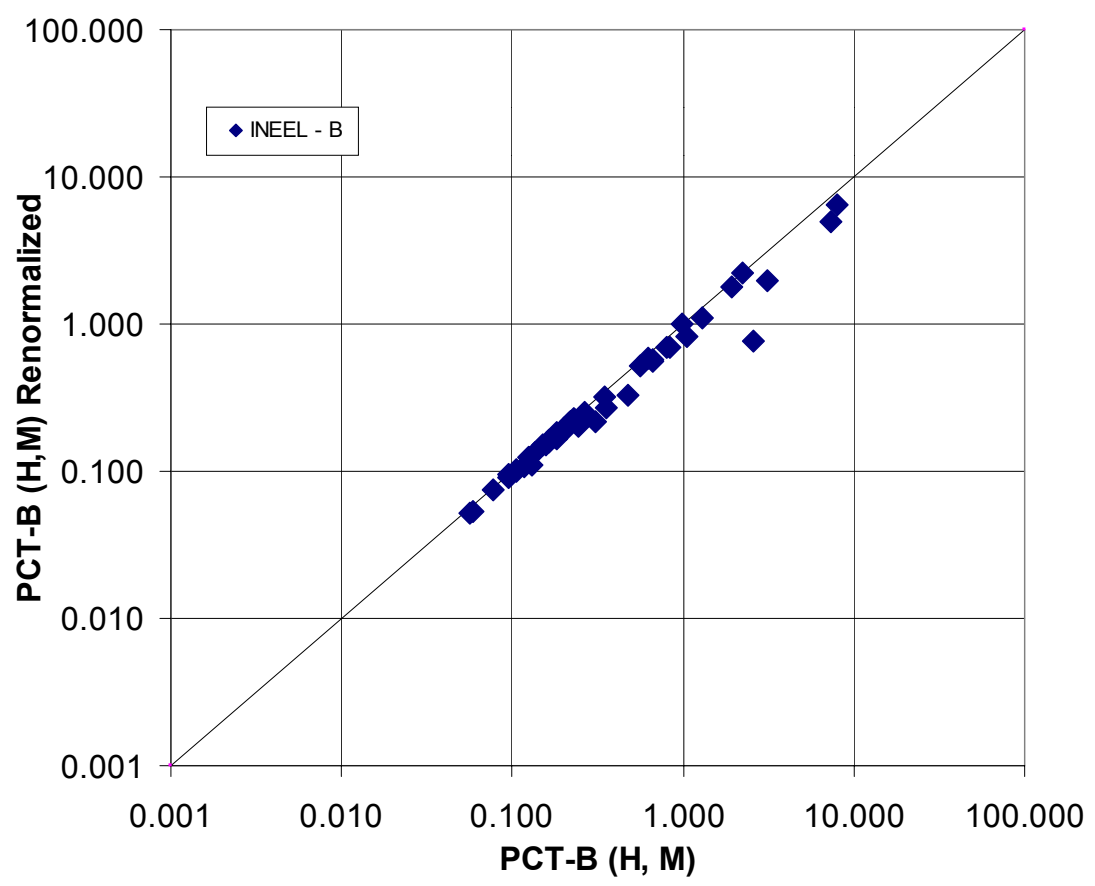

Figure A-23. INEEL Glasses: $r_{h b}$, Residual vs. Original

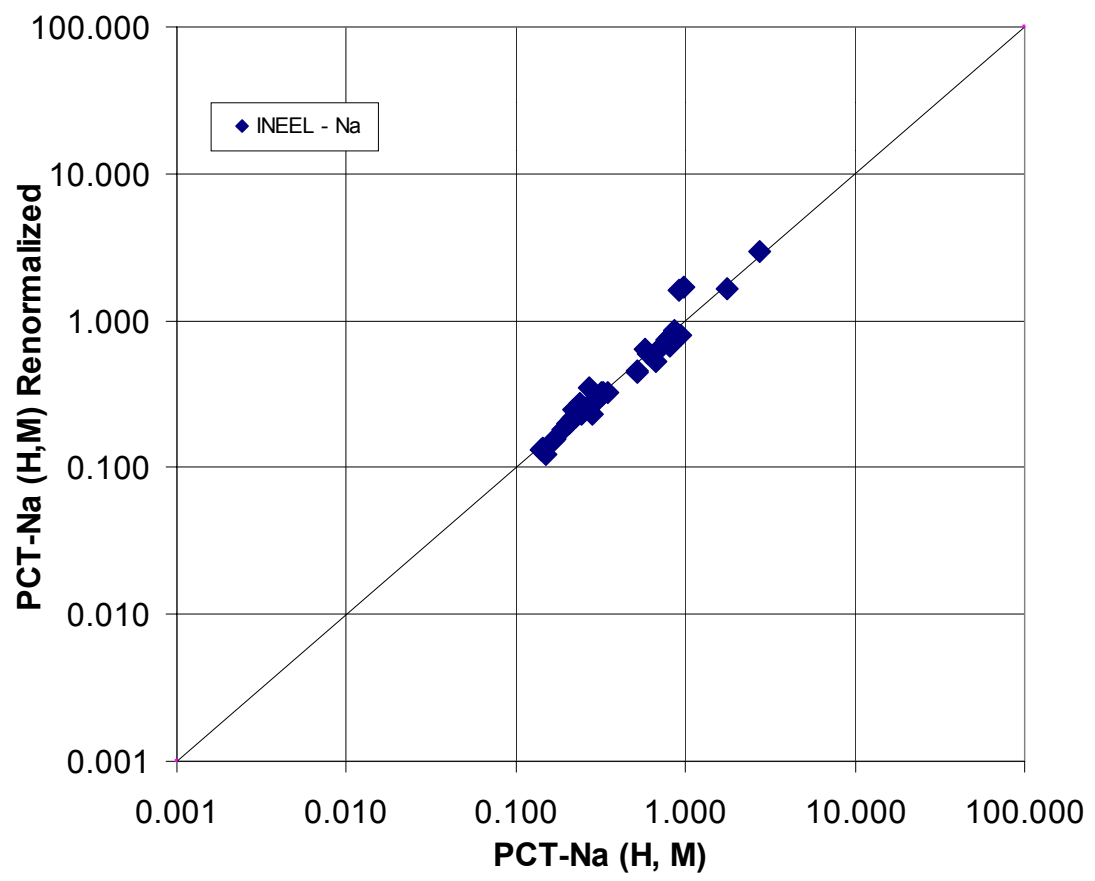

Figure A-24. INEEL Glasses: $r_{h N a}$, Residual vs. Original
A.30 
PNNL-13491

\section{Distribution}

No. of

Copies

\section{OFFSITE}

1 Professor Ian Pegg

Catholic University of America

Vitreous State Laboratory

620 Michigan Ave., N. E.

Washington, D. C. 20064

1 Brian Riley

1 Jose Rosario

5 Westinghouse Savannah River Company Aiken, South Carolina 29808

Attn:

K. G. Brown

E. W. Holtzscheiter

C. M. Jantzen

S. L. Marra

D. K. Peeler
704-1T

773-A

773-A

773-A

$773-43 \mathrm{~A}$
No. of

Copies

\section{ONSITE}

2 DOE/Office of River Protection

N. R. Brown H6-60

E. J. Cruz H6-60

2 DOE/Richland Operations Office

T. E. Pietrok

$\mathrm{K} 8-50$

B. M. Mauss

$\mathrm{K} 8-50$

4 CH2M Hill Hanford Group

T. W. Crawford

R3-73

K. A. Gasper

L4-07

J. O. Honeyman

H6-18

D. J. Swanberg

$\mathrm{H} 4-02$

1 Numatec Hanford Company

S. L. Lambert

R3-75

38 Pacific Northwest National Laboratory

$\begin{array}{ll}\text { B. R. Allen } & \text { K6-24 } \\ \text { W. F. Bonner (5) } & \text { K9-14 } \\ \text { W. C. Cosby } & \text { K7-62 } \\ \text { J. V. Crum } & \text { K6-24 } \\ \text { P. R. Hrma (10) } & \text { K6-24 } \\ \text { D. S. Kim } & \text { K6-24 } \\ \text { E. V. Morrey } & \text { P7-28 } \\ \text { J. M. Perez } & \text { H6-61 } \\ \text { L. M. Peurrung } & \text { K6-24 } \\ \text { G. F. Piepel } & \text { K5-12 } \\ \text { M. J. Schweiger } & \text { K6-24 } \\ \text { J. D. Vienna (3) } & \text { K6-24 } \\ \text { J. H. Westsik } & \text { K9-91 } \\ \text { B. J. Williams - TFA (8) } & \text { K9-69 } \\ \text { Technical Report Files (2) } & \text { P8-55 }\end{array}$

